\title{
A Procedure for the Qualitative Interpretation of Fuel Centerline Thermocouple Response to Step- Power Decreases
}
M. E. Cunningham
D. D. Lanning
S. D. Montgomery

October 1979

Prepared for

the U.S. Nuclear Regulatory Commission

Pacific Northwest Laboratory

Operated for the U.S. Department of Energy

by Battelle Memorial Institute

\section{5attelle}


NOTICE

This report was prepared as an account of work sponsored by the United States Government. Neither the United States nor the Department of Energy, nor any of their employees, nor any of their contractors, subcontractors, or their employees, makes any warranty, express or implied, or assumes any legal liability or responsibility for the accuracy, completeness or usefulness of any information, apparatus, product or process disclosed, or represents that its use would not infringe privately owned rights.

The views, opinions and conclusions contained in this report are those of the contractor and do not necessarily represent those of the United States Government or the United States Department of Energy.

\author{
PACIFIC NORTHWEST LABORATORY \\ operated by \\ BATTELLE \\ for the \\ UNITED STATES DEPARTMENT OF ENERGY \\ Under Contract EY-76-C-06-1830
}
Printed in the United States of America
Available from
National Technical Information Service
United States Department of Commerce
5285 Port Royal Road
Springfield, Virginia 22151

Price: Printed Copy \$

*; Microfiche $\$ 3.00$

$\begin{array}{cc}\text { •Pages } & \begin{array}{c}\text { NTIS } \\ \text { Selling Price }\end{array} \\ 001-025 & \$ 4.00 \\ 026-050 & \$ 4.50 \\ 051-075 & \$ 5.25 \\ 076-100 & \$ 6.00 \\ 101-125 & \$ 6.50 \\ 126-150 & \$ 7.25 \\ 151-175 & \$ 9.00 \\ 176-200 & \$ 9.00 \\ 201-225 & \$ 9.25 \\ 226-250 & \$ 9.50 \\ 251-275 & \$ 10.75 \\ 276-300 & \$ 11.00\end{array}$


PNL -3096

NUREG/CR-1012

$\mathrm{R}-3$

A PROCEDURE FOR THE QUALITATIVE INTERPRETATION OF FUEL CENTERLINE THERMOCOUPLE RESPONSE TO STEP-POWER DECREASES
M. E. Cunningham
D. D. Lanning
S. D. Montgomery

October 1979

Prepared for the U.S. Nuclear Regulatory Commission Under a Related Services Agreement with the U.S.

Department of Energy under Contract EY-76-C-06-1830 Fin No. B-2043

Pac if ic Northwest Laboratory

Richland, Washington 99352 
-

.

. 


\section{ABSTRACT}

This report reviews the present calculational techniques that may be used to interpret the transient response of fuel centerline thermocouples to step decreases in rod power. A new technique developed herein involves plotting the natural logarithm of the normalized thermocouple data versus time, plotting various calculations in the same way, and observing the curvature of the resulting lines. Also described is the small computer code, MWRAM, which facilitates testing various models against transient data.

Transient data from IFA-513 is presented. This test assembly in the Halden Reactor, Norway, is jointly sponsored by the Nuclear Regulatory Commission and the Halden Project. A comparison of MWRAM calculations with this data has shown that fuel cracking appears to greatly influence the heat transfer modes in the fuel rod. A method of estimating the effective fuel-cladding gap size from this transient data is also discussed in this report. 
 


\section{SUMMARY AND CONCLUSIONS}

This report shows that the transient response of fuel centerline thermocouples is potentially definitive as to the partition of thermal resistance in the fuel rod between gap resistance and fuel resistance. However, meaningful data analysis is shown to be possible only if numerical (computer) calculations are carried out that account for the gross effects stemming from the temperature dependence of the thermal properties, as well as the more subtle effects of flux depression and energy storage in the cladding. In this regard, a computer program for solving the transient radial heat transfer equations for the fuel rod is presented herein. This program, MWRAM, is neither difficult nor expensive to run.

Two-dimensional effects (flux tilt and pellet eccentricity) are shown not to have a large effect on transient centerline temperature behavior. Thus, conclusions drawn from a radial heat transfer analysis of centerline temperature are valid, but must be understood to apply to azimuthally averaged temperatures.

Current transient data from 20\% step-power drops in the Halden Reactor have been examined and included. This data is sufficient to establish that, for $20 \%$ power decreases, the fuel rod maintains a constant gap size. The gap does not widen from pellet shrinkage as might be expected. However, during the work leading to this report the current data proved to be too "rough" to make a quantitative statement about the apparent gap size(a). Only a qualitative range could be determined.

In conclusion, although current transient fuel temperature data frustrates quantitative analysis, the procedures and numerical techniques we have developed for analyzing that data appear to be both valid and adequate.

(a) The transition from one steady state to the next was not sharp enough nor clean enough, in general. 


\section{CONTENTS}

ABSTRACT

SUMMARY AND CONCLUSIONS

FIGURES

TABLES

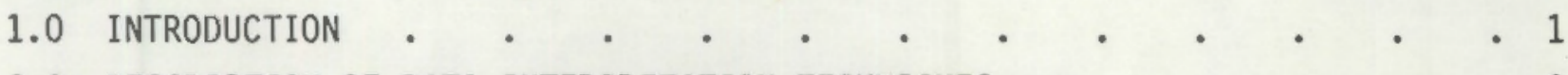

2.0 DESCRIPTION OF DATA INTERPRETATION TECHNIQUES.$\quad \cdot \quad \cdot \quad \cdot \quad \cdot 3$

LUMPED PARAMETER SOLUTIONS.$\quad \cdot \quad \cdot \quad \cdot \quad \cdot \quad \cdot \quad \cdot \quad \cdot 3$

The Constant Parameter Model . . . . . . . . 3

The Solution for Temperature-Dependent Conductance . . . 4

The Effect of Finite Thermocouple Time Constant . . . . 12

Summary of Lumped-Parameter Solutions . • • • . . 21

DISTRIBUTED PARAMETER SOLUTIONS $. \quad . \quad . \quad . \quad . \quad . \quad . \quad .21$

Constant-Parameter Model (Cladding Excluded) . . . . . 23

NUMERICAL CALCULATIONS $. \quad . \quad . \quad . \quad . \quad . \quad . \quad . \quad . \quad .30$

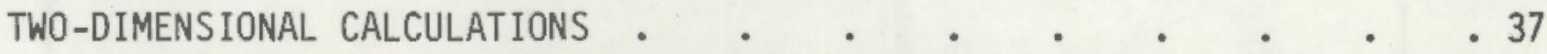

Effect of Eccentricity on Mean Fuel Center Temperature . . . 39

The Effects of Planar Flux Tilt . . . . . . . . 41

3.0 SAMPLE DATA INTERPRETATION $. \quad . \quad . \quad . \quad . \quad . \quad . \quad . \quad .45$

MODEL 1/MODEL 2 COMPARISONS $. \quad . \quad . \quad . \quad . \quad . \quad . \quad . \quad .47$

SURVEY CALCULATIONS • $. \quad . \quad . \quad . \quad . \quad . \quad . \quad . \quad . \quad .55$

EXTENSION TO OTHER IFA-513 THERMOCOUPLES $. . . \quad . \quad . \quad . \quad .66$

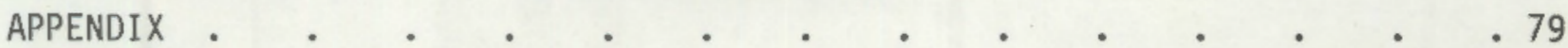

REFERENCES . . . . . . . . . . . . . . 103 


\section{FIGURES}

1a. Analytical and Numerical Lumped-Parameter Calculations for a High-Resistance Rod

1b. Analytical and Numerical Lumped-Parameter Calculations for a Low-Resistance Rod

2a. Residuals for the Various Calculations Applied to the High-Resistance Rod .

2b. Residuals of the Various Calculations Applied to the Low-Resistance Rod

3a. Effects of Different Thermocouple Time Constants in the High-Resistance Rod (Using the Lumped-Parameter

Fuel Model)

3b. Effects of Different Thermocouple Time Constants in the Low-Resistance Rod (Using the Lumped-Parameter Mode1)

4a. Results of Figure $3 a$, Replotted in the $\ln \left(T_{N}-a\right)$ Form

4b. Results of Figure $3 b$, Replotted in the $\ln \left(T_{N^{-a}}\right)$ Form

5a. Results of Different Thermocouple Time Constants in the High-Resistance Rod (Using Numerical Solution)

5b. Effects of Different Thermocouple Time Constants in the Low-Resistance Rod (Numerical Solution) . . . . . . . 20

6a. Effects of Difference Resistant Partitions in the High-Resistance Rod.

6b. Effects of Different Resistance Partitions in the Low-Resistance Rod

7. Resistance versus Power for the High-Resistance Rod, as Deduced from Initial/Final Steady-State Conditions

8. Model 1 and Model 2 Predictions for a $20 \%$ Power Decrease in the High-Resistance Rod

9. Effects of Different Assumed Conductances on the Model 2 Prediction for the High-Resistance Rod . . . . . . . 36

10. Two-Dimensional Mesh for TRUMP Calculations . . . . . . . 38

11. Response of Concentric and Eccentric Pellets to a $100 \%$ Power Decrease (TRUMP Calculations) 
12. Calculated Response of a 3-mil Hot Gap Rod (to a $20 \%$ Power Decrease) With and Without a 40\% Flux Tilt . . . . . . 42

13. Relative Heat Generation, Given Flux Depression Combined With Flux Tilt.

14. Response of Fuel Centerline Temperature to a Step 20\% Power Decrease, With and Without Flux Tilt . . . . . . . 44

15. The Thermal Resistance of Rod 6, IFA-513 (Upper Thermocouple) as a Function of Burnup . . . . . . . . . . 46

16. Unnormalized Temperature and Cobalt Detector Signal for Rod 6 (Upper Thermocouple) - Run 115 . . . . . . . . 48

17. Steady-State Temperature Versus Power for IFA-513, Rod 6, (Upper Thermocouple-TF-12) on First Startup . . . . . . 49

18. Relative Power History for the Transient Runs . . . . . 50

19. Data and Calculated Temperature Responses for the Upper Thermocouples of Rod 6 . . . . . . . . . . 51

20. Data and Calculated Temperature Response for Rod 6, Upper Thermocouple, Run 126

21. Data and Calculated Response for Rod 6 Upper, Thermocouple, Run 141

22. Comparison of Model 1 and Model 2 Against Rod 6 Data from Run 141

23. Model 2 Compared to Rod 6 Data for Run 115 (Assuming Gap Conductance $=2.0 \mathrm{~kW} / \mathrm{m}^{2}-{ }^{\circ} \mathrm{C}$ )

24. Model 2 Compared to Rod 6 Data from Run 115 (Assuming Gap Conductance $=3.0 \mathrm{~kW} / \mathrm{m}^{2}{ }^{\circ}{ }^{\circ} \mathrm{C}$ )

25. Model 2 Compared to Rod 6 Data from Run 115 (Assuming Gap Conductance $=4.0 \mathrm{~kW} / \mathrm{m}^{2}{ }^{\circ} \mathrm{C}$ )

26. Model 2 Compared to Rod 6 Data From Run 115 (Assuming Gap Conductance $=6.0 \mathrm{~kW} / \mathrm{m}^{2}-{ }^{\circ} \mathrm{C}$ )

27. Mode 12 Compared to Rod 6 Data from Run 115 (Assuming Gap Conductance $=8.0 \mathrm{~kW} / \mathrm{m}^{2}-{ }^{\circ} \mathrm{C}$ )

28. Model 2 Compared to Rod 6 Data from Run 126 (Assuming Gap Conductance $=2.0 \mathrm{~kW} / \mathrm{m}^{2}-{ }^{\circ} \mathrm{C}$ ) 
29. Model 2 Compared to Rod 6 Data from Run 126

(Assuming Gap Conductance $=3.0 \mathrm{~kW} / \mathrm{m}^{2}-{ }^{\circ} \mathrm{C}$ ) . $. \quad . \quad .59$

30. Mode 12 Compared to Rod 6 Data for Run 126

(Assuming Gap Conductance $=4.0 \mathrm{~kW} / \mathrm{m}^{2}-{ }^{\circ} \mathrm{C}$ )

31. Model 2 Compared to Rod 6 Data from Run 126

(Assuming Gap Conductance $=6.0 \mathrm{~kW} / \mathrm{m}^{2}-{ }^{\circ} \mathrm{C}$ )

32. Mode1 2 Compared to Rod 6 Data from Run 126 (Assuming Gap Conductance $=8.0 \mathrm{~kW} / \mathrm{m}^{2}-{ }^{\circ} \mathrm{C}$ )

33. Model 2 Compared to Rod 6 Data from Run 141 (Assuming Gap Conductance $=2.0 \mathrm{~kW} / \mathrm{m}^{2}{ }^{\circ} \mathrm{C}$ )

34. Mode 12 Compared to Rod 6 Data from Run 141 (Assuming Gap Conductance $=3.0 \mathrm{~kW} / \mathrm{m}^{2}-{ }^{\circ} \mathrm{C}$ )

35. Model 2 Compared to Rod 6 Data from Run 141 (Assuming Gap Conductance $=4.0 \mathrm{~kW} / \mathrm{m}^{2}-{ }^{\circ} \mathrm{C}$ )

36. Mode 12 Compared to Rod 6 Data from Run 141 (Assuming Gap Conductance $=6.0 \mathrm{~kW} / \mathrm{m}^{2}-{ }^{\circ} \mathrm{C}$ )

37. Model 2 Compared to Data from Run 141

(Assuming Gap Conductance $=8.0 \mathrm{~kW} / \mathrm{m}^{2}{ }^{\circ} \mathrm{C}$ ) . . . . 65

38. Model 2 Compared to Data from Run 141 (Gap Conductance = $\left.8.0 \mathrm{~kW} / \mathrm{m}^{2}-{ }^{\circ} \mathrm{C}\right)$ Assuming Thermocouple Time Constant = $1 / 0.2 \mathrm{sec}^{-1}$. . . . . . . . . . . 67

39. Models 1 and 2 Compared to Rod 1 (Lower Thermocouple Data for Run 141) . . . . . . . . . . 68

40. Models 1 and 2 Compared to Rod 1 Data (Upper Thermocouple) from Run 141

41. Models 1 and 2 Compared to Rod 2 Oata (Lower Thermocouple) for Run 141

42. Models 1 and 2 Compared to Rod 3 Data (Lower Thermocouple) for Run 141

43. Models 1 and 2 Compared to Rod 4 Data (Lower Thermocouple) for Run 141

44. Models 1 and 2 Compared to Rod 4 Data (Upper Thermocouple) for Run 141 
45. Models 1 and 2 Compared to Rod 5 Data (Lower Thermocouple) for Run 141

46. Models 1 and 2 Compared to Rod 5 Data (Upper Thermocouple) for Run 141

47. Models 1 and 2 Compared to Rod 6 Data (Lower Thermocouple) from Run 141

\section{TABLES}

1. Numerical Values for Comparative Lumped Parameter Calculations . . 9

2. Possible Partitions of Resistance for the Data in Table $1 \quad$ • $\quad$ - 27

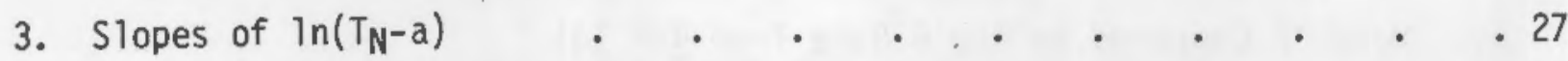

4. Input Parameters for Comparative MWRAM Calculations

(High Resistance Rod) . . . . . . . . . . . . 33

5. Results of Various Choices of Gap Conductances (Model 2,

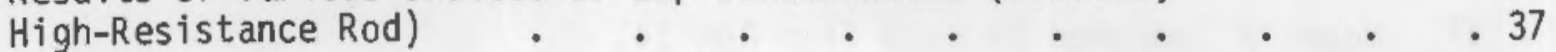

6. Major Input Parameters for Two-Dimensional Comparative Cases . . 39

7. Effect of Flux Tilt with Flux Depression (Using Parameters in Table 5) . $5 . \quad . \quad . \quad . \quad . \quad . \quad . \quad . \quad . \quad 44$

8. Initial/Final Conditions and MWRAM Parameters for TF 12
(Rod 6 Upper Thermocouple) . . . . . . . . . . 48

9. Crack Factors for Model 2 for TF 12 Using Specific Values

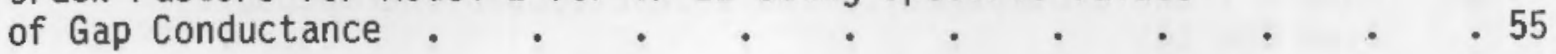

10. Thermocouple Positions and Fill Gas Compositions for IFA-513 Fuel Rods 


\subsection{INTRODUCTION}

The transient response of a fuel centerline thermocouple to a small step decrease in rod power is potentially definitive as to the distribution of thermal resistance within the fuel rod. In particular, the partition of resistance between fuel and gap may be better defined from the transient data.

The need for improved experimental definition of the partition of thermal resistance within fuel rods was pointed out in an earlier report. (1) In that report we showed, in effect, that centerline temperature versus power data can be explained by constrained combinations of fuel and gap resistance spanning the full range of physical possibility for each resistance separately. Yet predicted transient cladding behavior during the loss-of-coolant accident is significantly affected by the resistance partition that is chosen. If one pays attention to the behavior of total resistance versus power data, one can narrow considerably the range of possible combinations. (1) But the range is still very large if one permits a temperature-dependent "crack factor" to modify the fuel thermal conductivity.

In this report, we show how the transient fuel centerline thermocouple response to step-power decreases can be used to at least qualitatively assess calculated fuel conductivity modifications and attendant gap conductance functions. The basis for this assessment is the fact that, even when constrained to match the same steady-state temperature versus power data, different models do not predict the same transient response.

Data for transient thermocouple response to $20 \%$ power decreases is becoming available. The data comes from test fuel assemblies in the Halden Reactor, Norway, which were designed for the Nuclear Regulatory Commission by Pacific Northwest Laboratory ${ }^{(a)}$. We have already reported some of this $\operatorname{data}^{(2)}$ and sketched the procedure by which it is used to infer the partition of thermal resistance. The associated analytical derivations and (a) Pacific Northwest Laboratory located in Richland, Washington, is operated
for the Department of Energy by Battelle Memorial Institute. 
numerical techniques are explained in detail in Section 2 of this report. Section 3 describes and documents the particular procedure we use for interpreting the transient thermocouple data. 


\subsection{DESCRIPTION OF DATA INTERPRETATION TECHNIQUES}

This section includes a discussion of the various analytical and numerical techniques that can be used to interpret the transient response of a fuel centerline thermocouple. Naturally, the more sophisticated the technique, the higher the complexity and cost become. The more idealized and simplistic solutions are presented, not because they are actually used, but because they are easy to understand and do reveal major effects that are common to the more complex calculations.

\section{LUMPED PARAMETER SOLUTIONS}

The simplest model for transient calculation is the lumped parameter model, wherein a section of the fuel rod is characterized by a single temperature, $\mathrm{T}$, total heat capacity, $\mathrm{C}\left(\mathrm{Joule} /{ }^{\circ} \mathrm{C}\right)$, and a fuel-to-coolant total conductance, $\mathrm{H}\left(\mathrm{W} /{ }^{\circ} \mathrm{C}\right)$. We will explore the predictions of this model for constant and temperature-dependent conductance, with and without the effects of a finite thermocouple time constant.

\section{The Constant Parameter Model}

In this, the simplest of all fuel rod models, values for $C$ and $H$ are fixed at their initial values, $\mathrm{C}_{0}$ and $\mathrm{H}_{0}$. The analytical solution to the transient heat transfer equation is exact. The transient equation is simply

$$
\frac{C_{0} d T}{d t}=-H_{0}\left(T-T_{C 001}\right)+Q(t)
$$

where $T_{\mathrm{CoO}}$ is coolant temperature and $Q(t)$ is heat generation (in watts).

The initial condition is $Q_{0}=H_{0}\left(T_{0}-T_{\text {cool }}\right)$.

For a step drop, 


$$
\begin{aligned}
Q(t) & =Q_{0}, t<0 \\
& =Q_{0}-\Delta Q, t>0
\end{aligned}
$$

If we let $T=T_{0}+\Delta T(t)$, substitute this into Equation 1 and eliminate steady-state terms, we have,

$$
C_{0} \frac{d \Delta T}{d t}=H \Delta T-\Delta Q
$$

The LaPlace transform of this equation is

$$
C_{0} S \Delta T^{\star}=-H \Delta T^{*}-\frac{\Delta Q}{S},
$$

where $\Delta T^{*}$ is the LaPlace transform of $\Delta T$. Solving for $\Delta T^{\star}$, and transforming,

$$
\Delta T=\frac{-\Delta Q}{b C_{0}}\left[1-e^{-b t}\right], b=H_{0} / C_{0}
$$

By defining $a=\left(Q_{0}-\Delta Q\right) / Q_{0}$ and $T_{N}=\left(T-T_{\text {cool }}\right) /\left(T_{0}-T_{\text {cool }}\right)$ Equation 3 transforms to

$$
T_{N}=a+(1-a) e^{-b t}
$$

\section{The Solution for Temperature-Dependent Conductance}

First of all, we will consider the effect of a temperature dependence for the lumped parameter conductance, since all the parameters of a real fuel rod 
are in fact temperature dependent. We will assume that $H$ is linearly dependent on $\mathrm{T}: \mathrm{H}=\mathrm{A}+\mathrm{BT}$. For this case we will derive an approximate analytical solution and a numerical algorithm. Several aspects will become clear from the results. In this case, the transient heat transfer equation (with $T_{\mathrm{COO}}=0$ ) is

$$
\frac{C d T(t)}{d t}=-(A+B T) T+Q(t)
$$

with initial condition $H_{0} T_{0}=T_{0}\left(A+B T_{0}\right)=Q_{0}$. Now let

$$
\begin{aligned}
& T(t)=T_{0}+\Delta T(t) \\
& Q(t)=Q_{0}-\Delta Q
\end{aligned}
$$

as before. Substitution into Equation 5 and elimination of steady-state terms yields a transient equation:

$$
\frac{C_{0} d T(t)}{d t}=-\Delta Q-\Delta T\left(A+2 B T_{0}+B \Delta T\right)
$$

with initial condition $\Delta T=0$ at $t=0$.

To derive an approximate analytical solution, we note that $2 T_{0} \gg \Delta T$ for $20 \%$ step power changes, and so we drop the $B \Delta T$ term in the parentheses. We apply LaPlace transforms to the reduced equation and solve for the transformed temperature component $\Delta T^{\star}$, as before, to obtain

$$
\Delta T^{*}=\frac{-\Delta Q / C_{0}}{(S+b) S}, b=\left(A+2 B T_{0}\right) / C_{0}
$$

Transforming to the time range, we have

$$
\Delta T=\frac{-\Delta Q}{C_{0}^{b}}\left[1-e^{-b t}\right]
$$


This can be translated to normalized temperature $T_{N}=T / T_{0}$, much as before. The result is

$$
T_{N}=1-\left[\frac{A+B T_{0}}{A+2 B T_{0}}\right](1-a)\left(1-e^{-b t}\right)
$$

A more exact solution to Equation 5 is a numerical algorithm, wherein the conductance for the current time step is estimated from the temperature of the previous time step. The time derivative is approximated by forward finite differencing as

$$
\frac{d T}{d t}=\frac{T^{n+1}-T^{n}}{\Delta t}
$$

where the superscripts $n+1$ and $n$ refer to the end and beginning of the time step, and $\Delta t$ is the time-step size. Equation 5 can then be solved for $T^{n+1}$ :

$$
T^{n+1}=\left(C T^{n}+\Delta t\left(Q_{0}-\Delta Q\right)\right) /\left(C+H^{n} \Delta t\right)
$$

Figure 1 shows results of the above equation for the input listed in Table 1 (for a high-resistance and a low-resistance rod). Note that a major difference in the two rods, in addition to the magnitude of the resistance, is the slope of the resistance versus power. The high-resistance rod has a sharply decreasing resistance versus power (positive conductance slope), which is typical of rods in light fuel-cladding contact at high burnup. In the low resistance rod, the resistance increases with power (negative conductance slope). This is typical of rods in firm contact. Results for several time step sizes are shown, as well as the results from the analytical solutions. The constant-parameter solution is clearly in error, since it has the wrong asymptotic value. But how do we assess the other solutions since all of them 


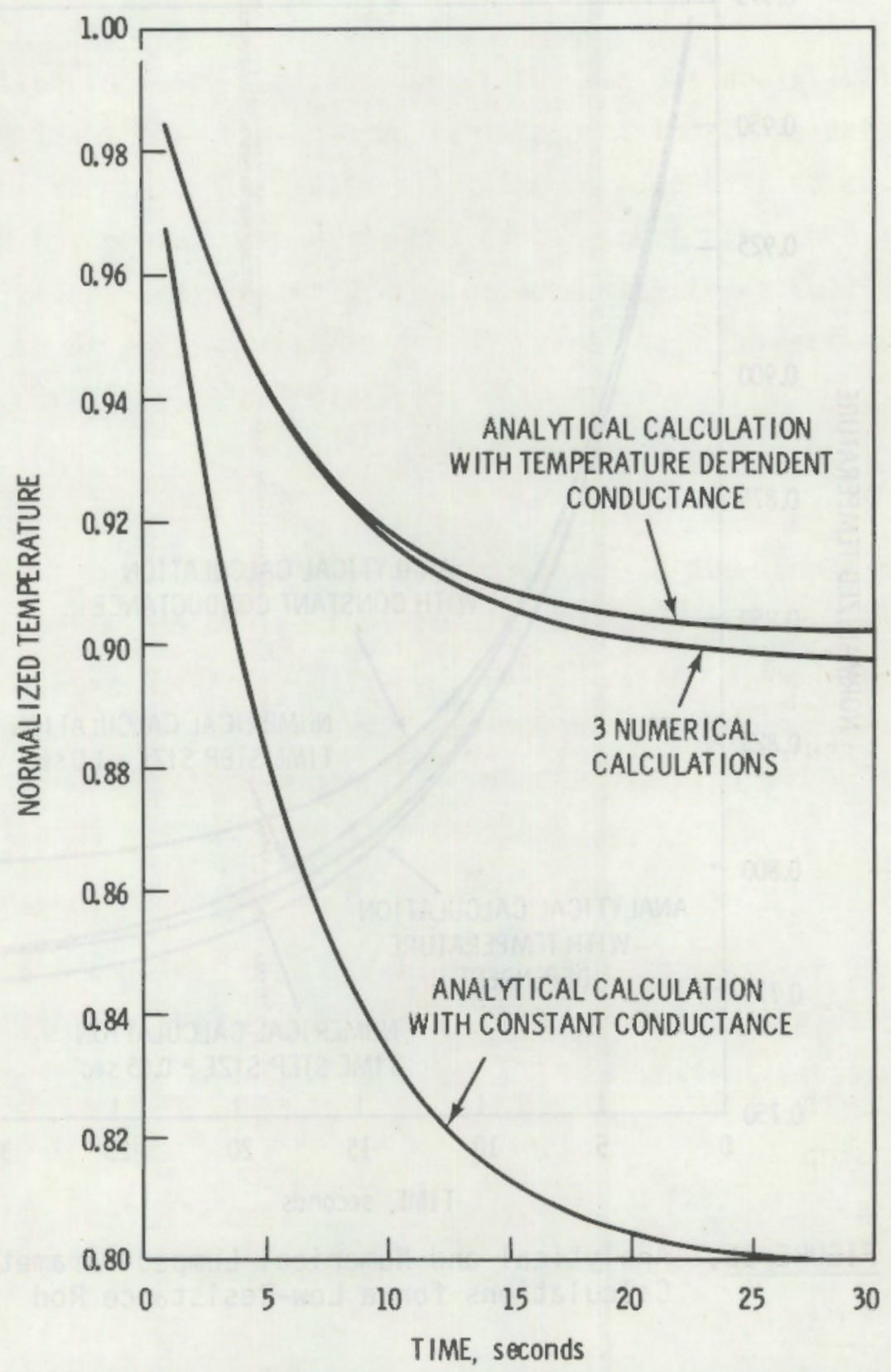

FIGURE 1a. Analytical and Numerical Lumped-Parameter Calculations for a High-Resistance Rod 


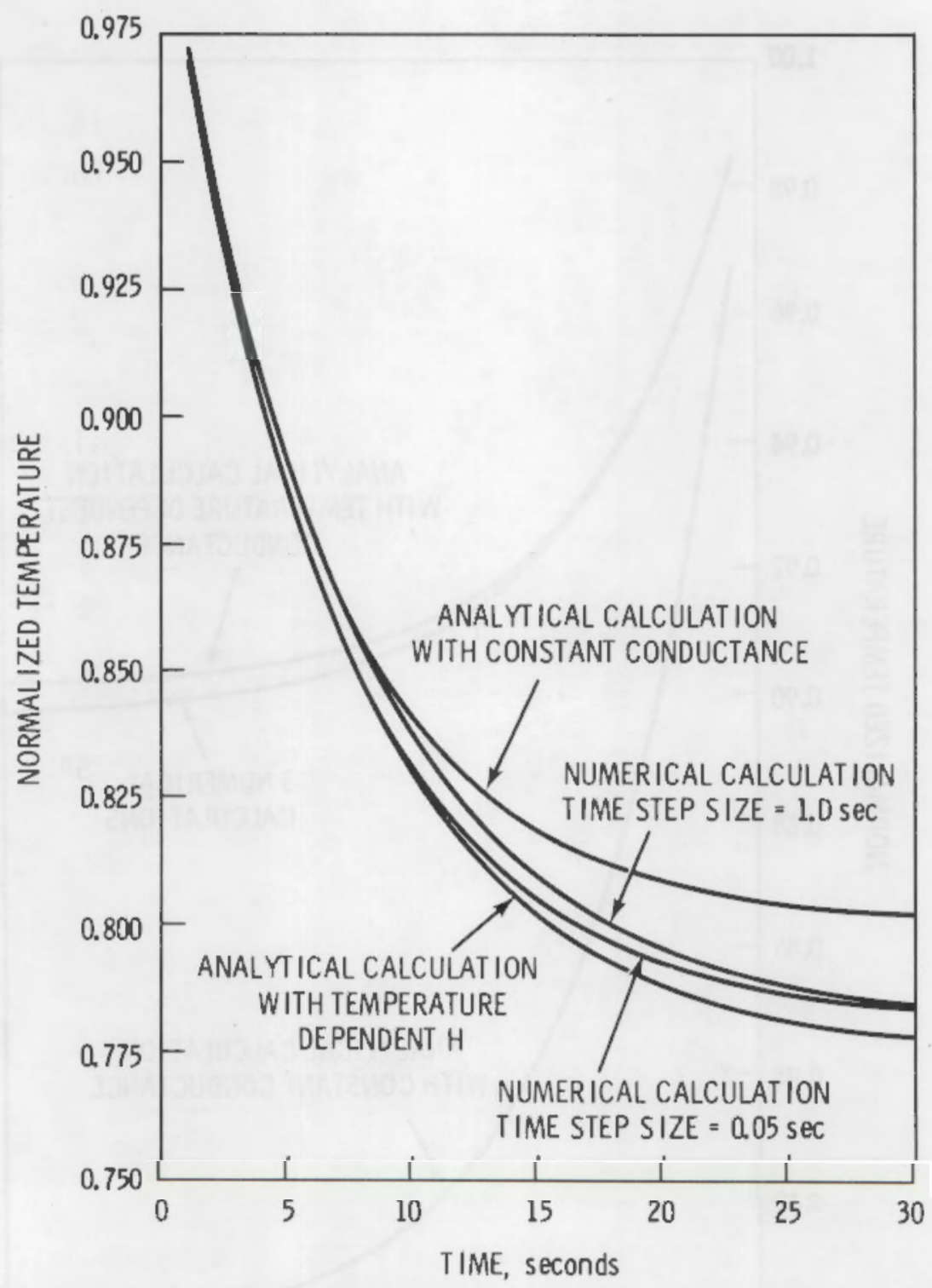

FIGURE 1b. Analytical and Numerical Lumped-Parameter Calculations for a Low-Resistance Rod 
are approximate? One way is to substitute them into Equation 5, which is exact, and note the residual (i.e., the inequality between right and left sides). Figure 2 shows the results for the temperature-dependent conductance cases. Two important observations may be made:

- For time-step sizes - less than 0.5 second, the analytical solution is less accurate than the numerical.

- Not much extra accuracy is gained for time-step sizes below 0.1 seconds. These conclusions are quite general, and applicable to models that are much more complicated. All numerical solutions referenced henceforth will have time-step sizes of $0.1 \mathrm{sec}$. TABLE 1. Numerical Values for Comparative Lumped
Parameter Calculations $($ a)

\begin{tabular}{|c|c|c|c|c|}
\hline Item & Symbol & $\begin{array}{l}\text { High Resistance } \\
\text { Rod Values } \\
\end{array}$ & $\begin{array}{l}\text { Low Resistance } \\
\text { Rod Values }\end{array}$ & Units \\
\hline Conductance Intercept & A & -0.00694 & 0.51834 & $w /{ }^{\circ} \mathrm{C}$ \\
\hline Conductance Slope & $B$ & $2.346 \mathrm{E}-4$ & $-5.376 E-5$ & $w /\left({ }^{0} \mathrm{C}\right)^{2}$ \\
\hline Heat Capacity & $c_{0}$ & 2.92 & 2.92 & joule $/{ }^{\circ} \mathrm{C}$ \\
\hline Initial Conductance & $\mathrm{H}_{\mathrm{O}}$ & 0.249 & 0.4654 & $\mathrm{~W} /{ }^{\circ} \mathrm{C}$ \\
\hline Initial Power & $Q_{0}$ & 272. & 458 & $W$ \\
\hline Power Change & $\Delta Q$ & 54.4 & 91.6 & $W$ \\
\hline $\begin{array}{l}\text { Initial Temperature } \\
\text { (above coolant) }\end{array}$ & $\mathrm{T}_{0}$ & 1091. & 984. & ${ }^{\circ} \mathrm{C}$ \\
\hline Coolant Temperature & $T_{\text {cool }}$ & 0 & 0 & ${ }^{\circ} \mathrm{C}$ \\
\hline
\end{tabular}

(a) These values apply to $0.01 \mathrm{~m}$ section of a fuel rod. 


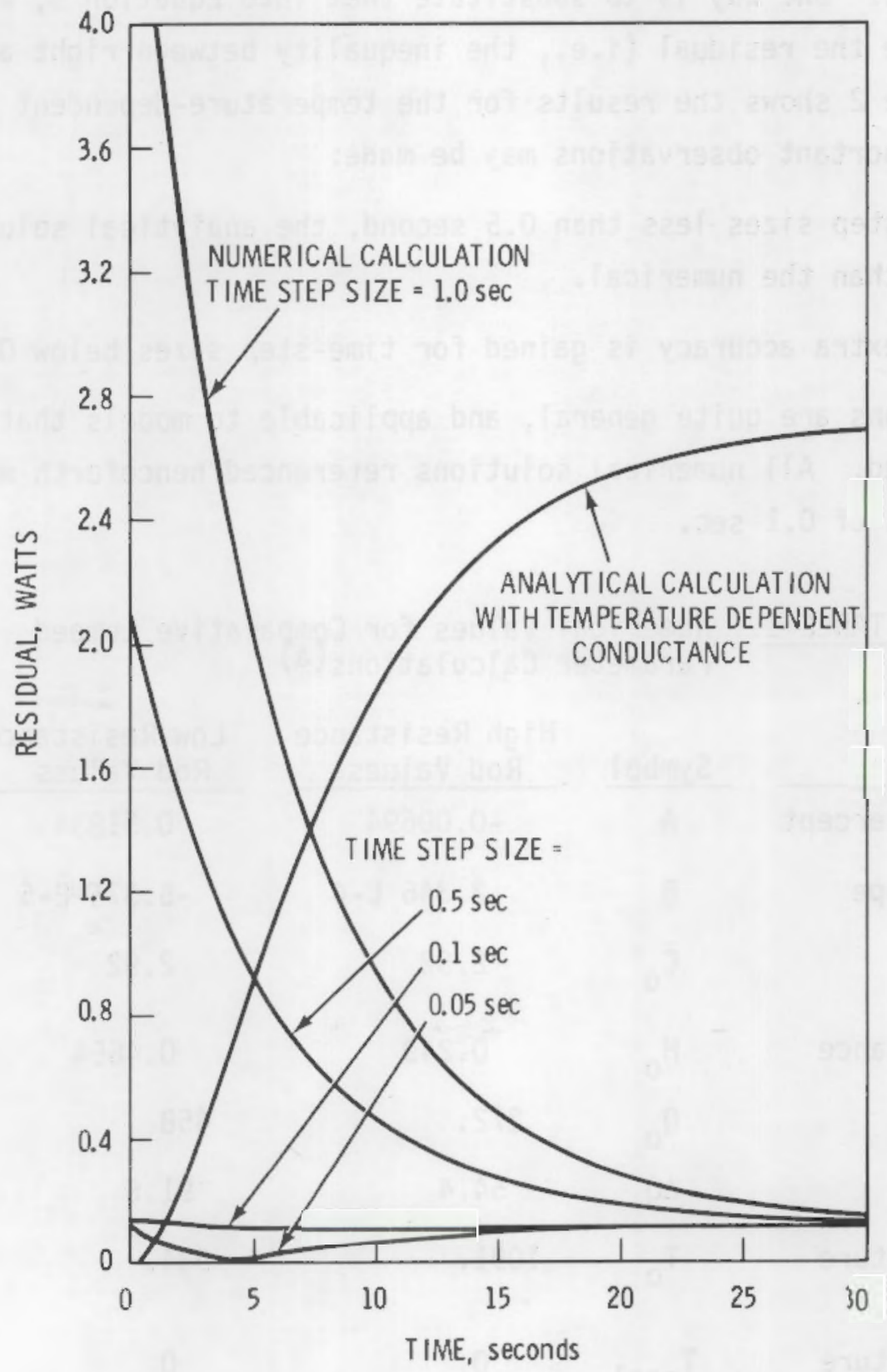

FIGURE 2a. Residuals for the Various Calculations Applied to the High-Resistance Rod 


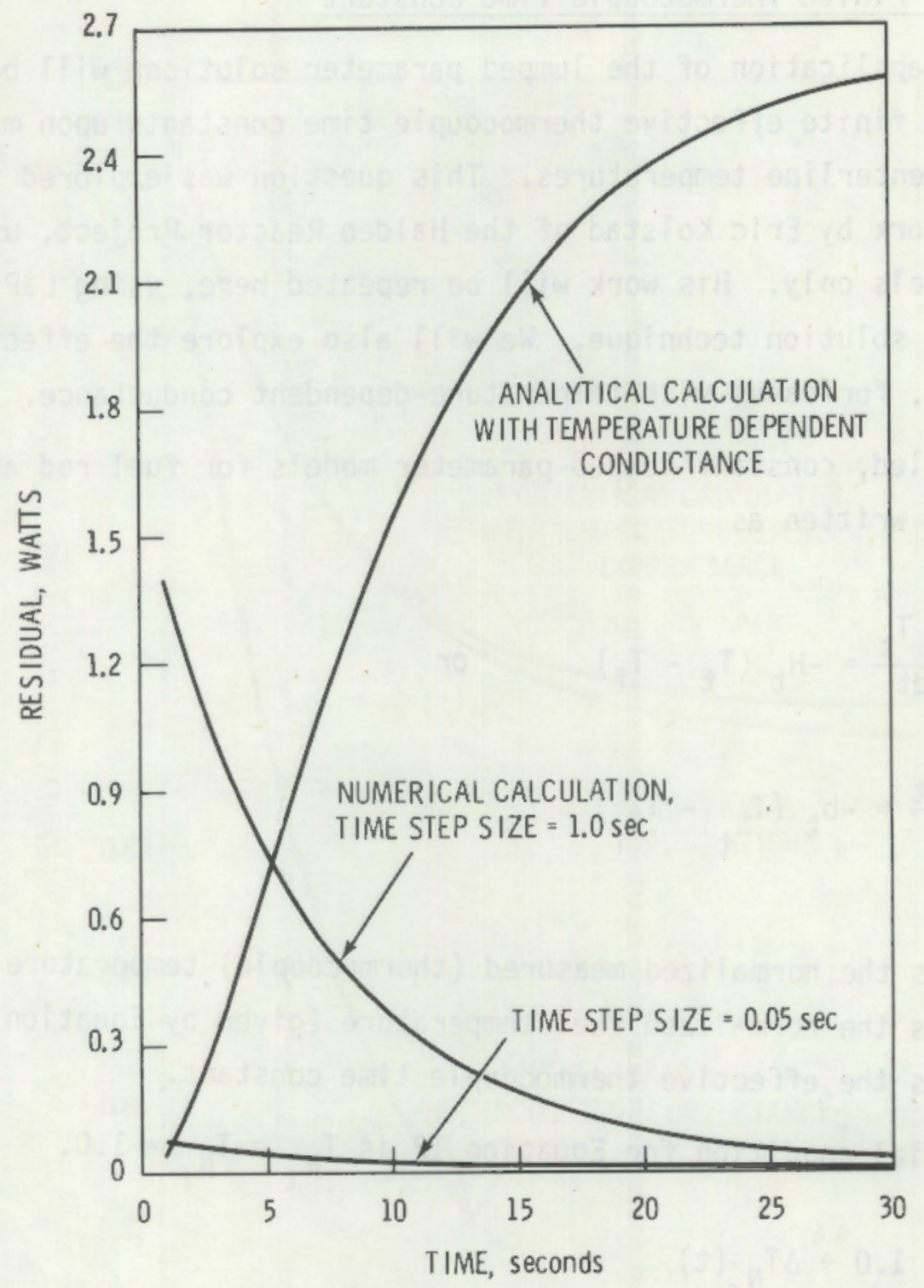

FIGURE 2b. Residuals of the Various Calculations Applied to the Low-Resistance Rod 
The Effect of Finite Thermocouple Time Constant

A final application of the lumped parameter solutions will be to assess the effect of finite effective thermocouple time constants upon measuredversus-true centerline temperatures. This question was explored thoroughly in unpublished work by Eric Kolstad of the Halden Reactor Project, using constant parameter models only. His work will be repeated here, using LaPlace transforms for the solution technique. We will also explore the effect, via numerical solution, for cases with temperature-dependent conductance.

The coupled, constant lumped-parameter models for fuel rod and thermocouple may be written as

$$
\begin{aligned}
& c_{t} \frac{d T_{t}}{d t}=-H_{t}\left(T_{t}-T_{f}\right) \quad \text { or } \\
& \frac{d T_{N_{t}}}{d t}=-b_{t}\left(T_{N_{t}}-T_{N_{f}}\right)
\end{aligned}
$$

where: $T_{N_{t}}$ is the normalized measured (thermocouple) temperature

$T_{N_{f}}^{t}$ is the normalized fuel temperature (given by Equation 4)

$b_{t}$ is the effective thermocouple time constant.

The initial condition for Equation 12 is $\mathrm{T}_{\mathrm{N}_{t}}=\mathrm{T}_{\mathrm{N}_{\mathrm{f}}}=1.0$.

$$
\text { Letting } \begin{aligned}
\mathrm{T}_{\mathrm{N}_{f}} & =1.0+\Delta \mathrm{T}_{\mathrm{N}_{\mathrm{f}}}(\mathrm{t}) \\
\mathrm{T}_{\mathrm{N}_{t}} & =1.0+\Delta \mathrm{T}_{\mathrm{N}_{t}}(t)
\end{aligned}
$$

and noting from Equation 4 that:

$$
\Delta T_{N_{f}}=-(1-a)\left[1-e^{-b_{f} t}\right] \text {, }
$$


Equation 12 can be recast as:

$$
\frac{d \Delta T_{t}}{d t}=-b t\left[\Delta T_{t}+(1-a)-(1-a) e^{-b} f^{t}\right]
$$

Taking the LaPlace transform of both sides and solving for $\Delta \mathrm{T}_{\mathrm{N}_{\mathrm{t}}}{ }^{*}$, we have

$$
\Delta T_{N_{t}^{*}}^{*}=b_{t}(1-a)\left[\frac{1}{S b_{t}}-\frac{1}{b_{t}\left(S+b_{t}\right)}-\frac{1}{\left(b_{t}-b_{f}\right)\left(S+b_{f}\right)}+\frac{1}{\left(b_{t}-b_{f}\right)\left(S+b_{t}\right)}\right]
$$

After transforming and rearranging terms, we have

$$
T_{N_{t}}=a+(1-a)\left[\left(\frac{b_{t}}{b_{t}-b_{f}}\right) e^{-b_{f} t}-\left(\frac{b_{f}}{\left(b_{t}-b_{f}\right)}\right) e^{-b_{t} t}\right]
$$

Kolstad carries out numerical calculations with Equation 15 to show the range of discrepancy between the true and measured centerline temperatures, for various combinations of $b_{t}$ and $b_{f}$. The maximum difference he predicts is about $20 \%$ of the initial/final steady-state difference. In the case of a $20 \%$ power decrease, this is about $40^{\circ} \mathrm{C}$, but for a full scram it is over $200^{\circ} \mathrm{C}$. Figure 3 shows how the magnitude and curvature of normalized temperature versus time are both affected by the choice of $b_{t}$, in a $20 \%$ step power decrease. Note that the effect is most significant in the low-resistance rod.

However, it is possible to interpret the centerline temperature data without knowing an exact value for the thermocouple effective time constant. Note that $b_{t}>b_{F}$, and after a few seconds, Equation 15 reduces to: 


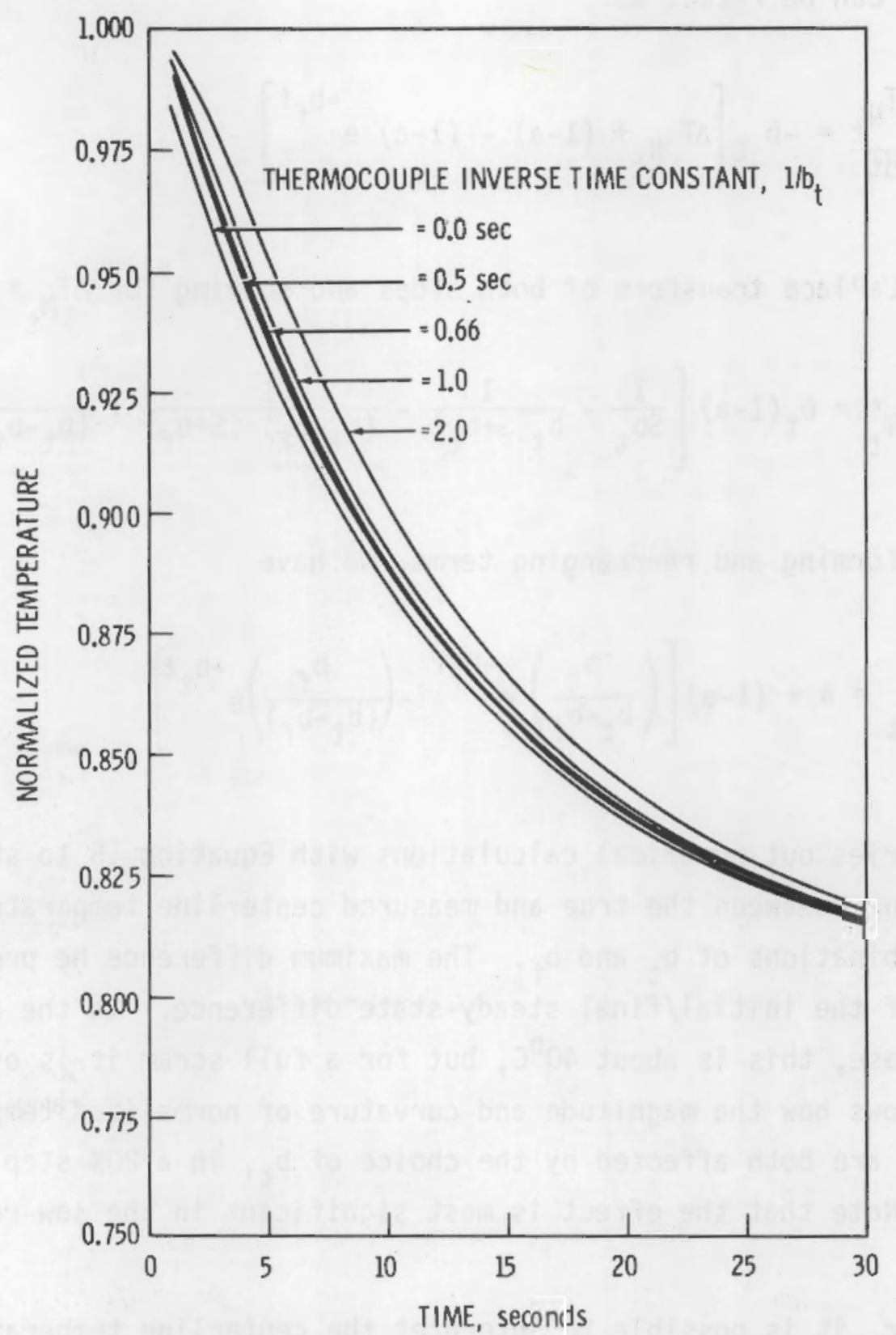

FIGURE 3a. Effects of Different Thermocouple Time Constants in the High-Resistance Rod (Using the Lumped-Parameter Fuel Mode1) 


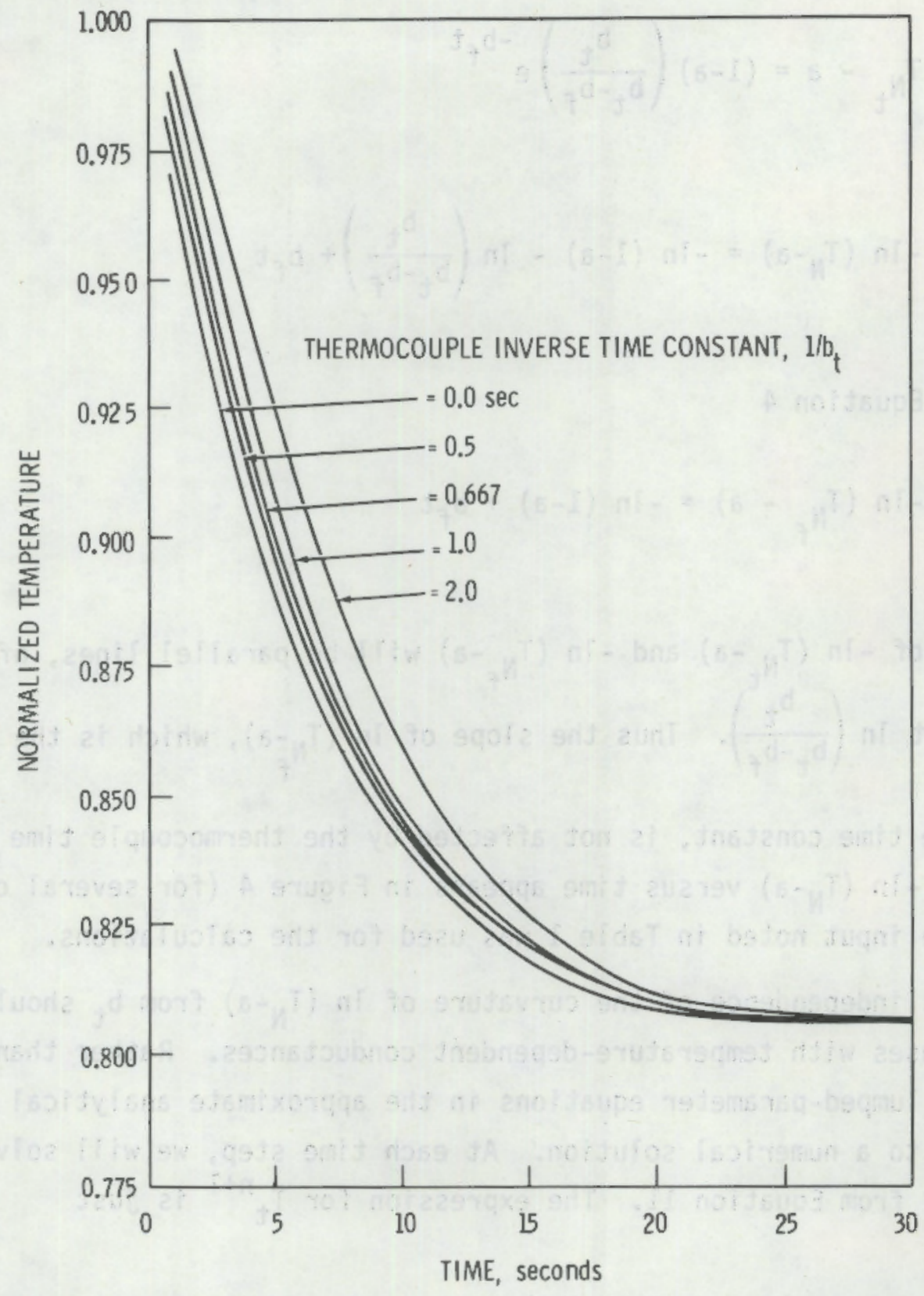

FIGURE 3b. Effects of Different Thermocouple Time Constants in the Low-Resistance Rod (Using the Lumped-Parameter Model) 


$$
T_{N_{t}}-a=(1-a)\left(\frac{b_{t}}{b_{t}-b_{f}}\right) e^{-b_{f} t}
$$

or

$$
-\ln \left(T_{N}-a\right)=-\ln (1-a)-\ln \left(\frac{b_{t}}{b_{t}-b_{f}}\right)+b_{f} t
$$

But from Equation 4

$$
-\ln \left(T_{N_{f}}-a\right)=-\ln (1-a)+b_{f} t
$$

So plots of $-\ln \left(T_{N_{t}}-a\right)$ and $-\ln \left(T_{N_{f}}-a\right)$ will be parallel lines, offset by the amount $\ln \left(\frac{b_{t}}{b_{t}-b_{f}}\right)$. Thus the slope of $\ln \left(T_{N_{f}}-a\right)$, which is the fuel

effective time constant, is not affected by the thermocouple time constant. A graph of $-\ln \left(T_{N^{-a}}\right.$ ) versus time appears in Figure 4 (for several choices of $\left.b_{t}\right)$. The input noted in Table 1 was used for the calculations.

This independence of the curvature of $\ln \left(T_{N}-a\right)$ from $b_{t}$ should carry over to the cases with temperature-dependent conductances. Rather than resolve the coupled, lumped-parameter equations in the approximate analytical manner, we skip directly to a numerical solution. At each time step, we will solve Equation 12 for $T_{t}{ }^{n+1}$ from Equation 11. The expression for $T_{t}{ }^{n+1}$ is just

$$
T_{t}^{n+1}=\left(b_{t} \Delta t T_{F}^{n+1}+T_{t}^{n}\right) /\left(1+b_{t} \Delta t\right)
$$

Results of computer calculations using Equations 11 and 18 are shown in Figure 5 , for several choices of $b_{t}$. The input from Table 1 was again 


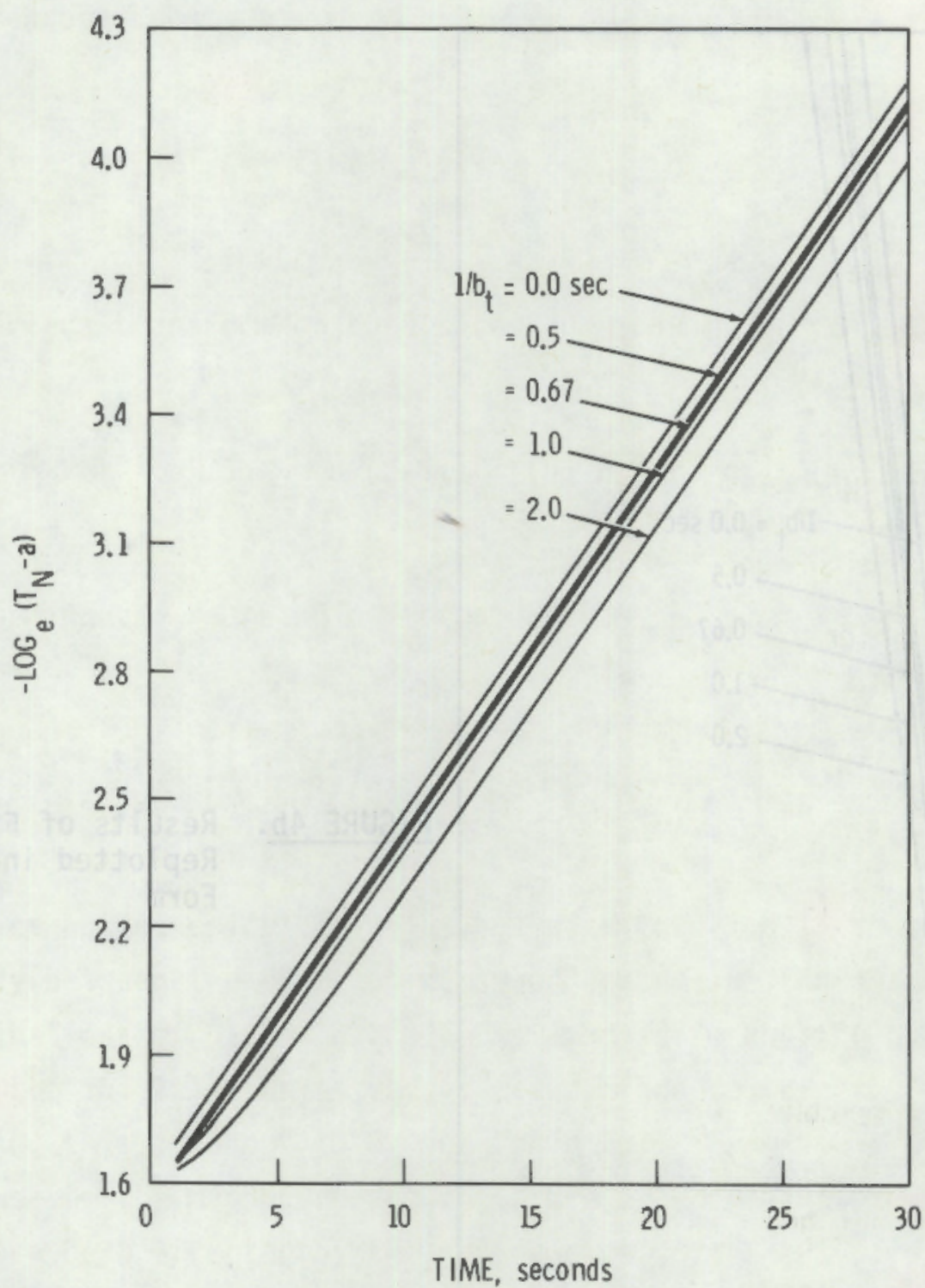

FIGURE 4a. Results of Figure $3 a$, Replotted in $\ln \left(T_{N}-a\right)$ Form 


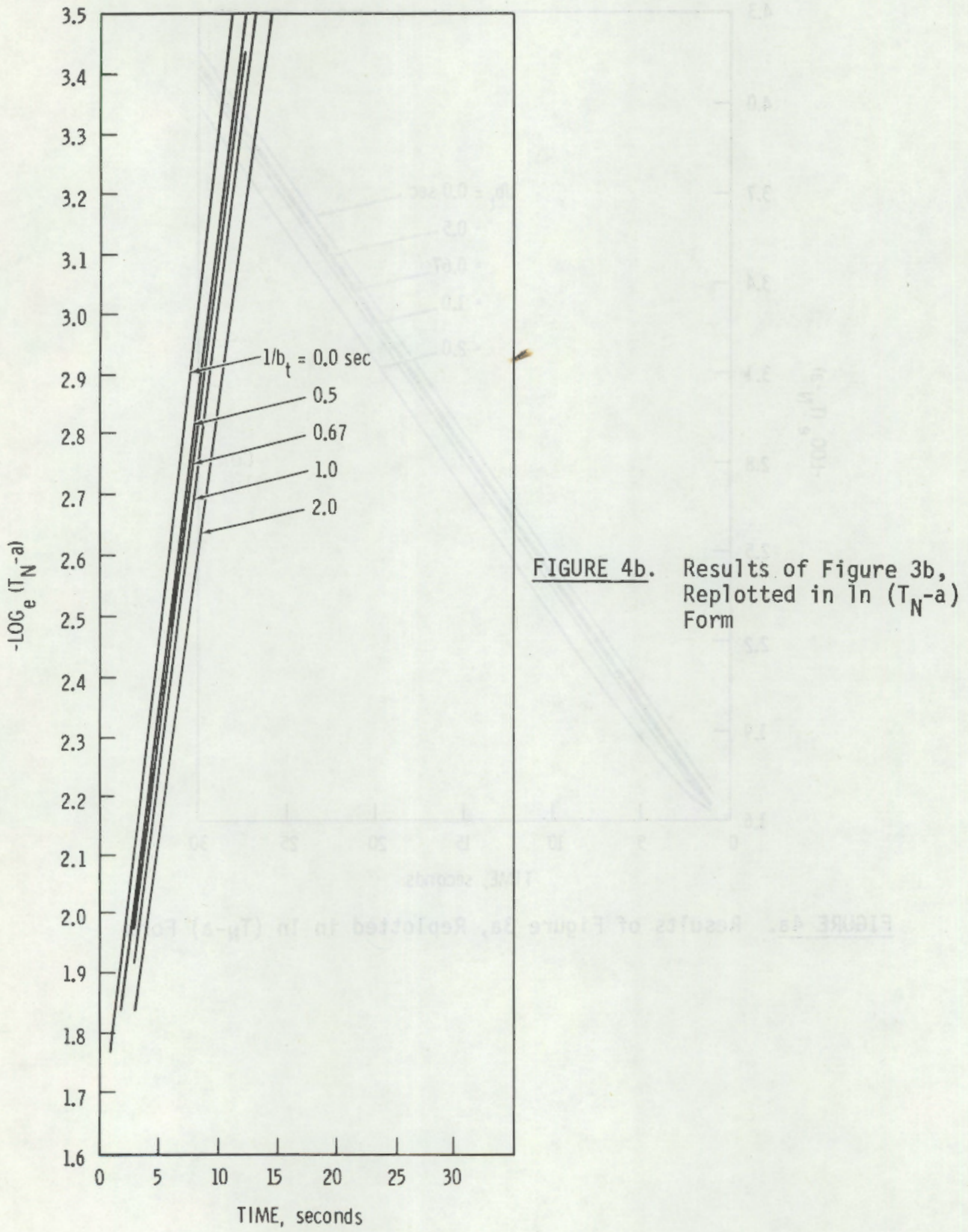




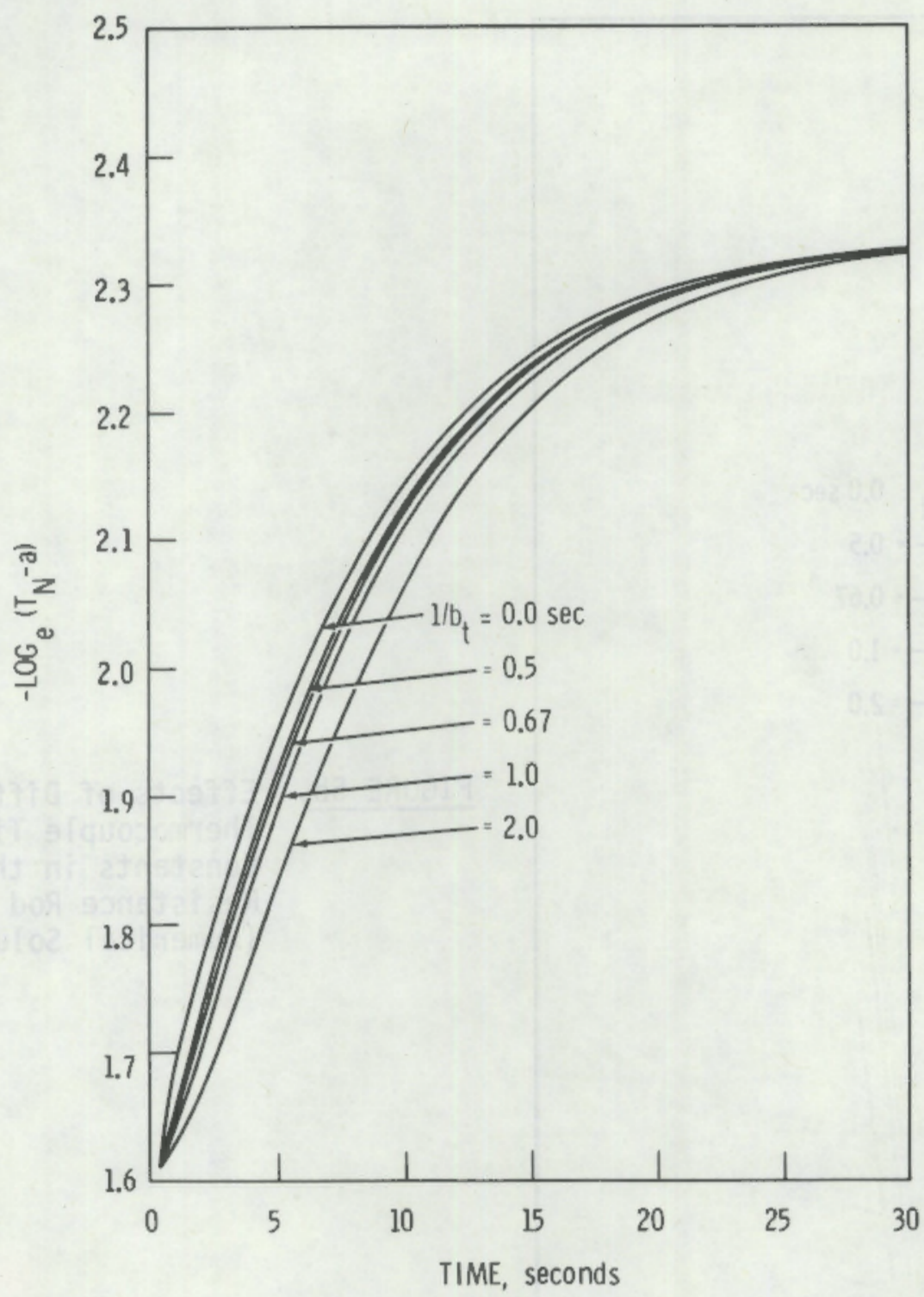

FIGURE 5a. Results of Different Thermocouple Time Constants in the High-Resistance Rod (Using Numerical Solution) 


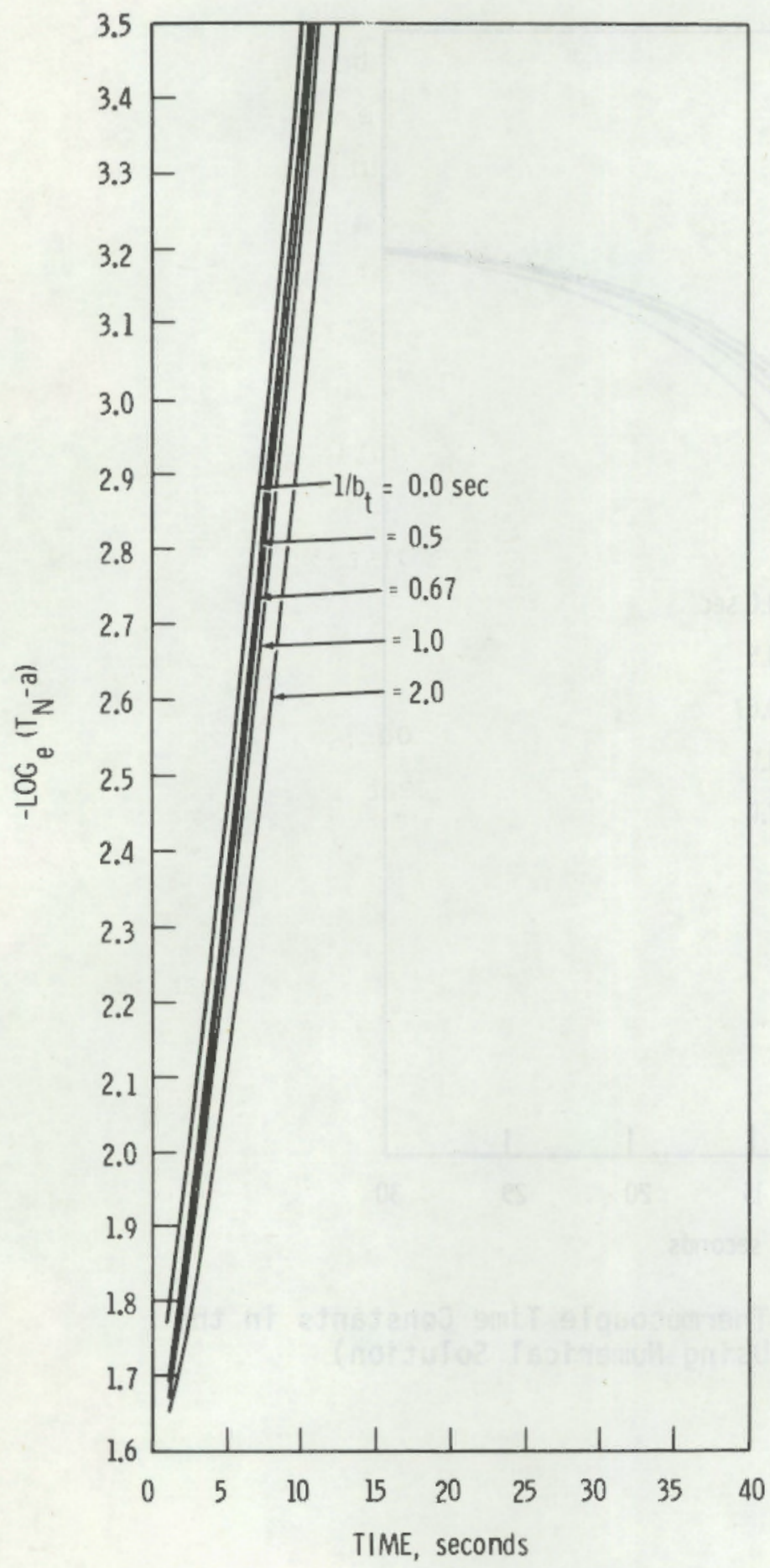

FIGURE 5b. Effects of Different Thermocouple Time Constants in the LowResistance Rod (Numerical Solution) 
used. One can see that over a broad time range, the curvature of the temperature plots remains unaffected by the choice of the thermocouple time constant. It is also clear from this plot that the curvature is different for cases where the slope of $H$ versus $T$ is positive rather than negative. This curvature is a qualitative confirmation of the resistance-versus-power behavior, and the relative steepness of the curves is qualitatively related to the relative magnitude of the resistances.

\section{Summary of Lumped-Parameter Solutions}

We have learned much from the lumped-parameter solutions. First, we have seen that, for significant variation of conductance with temperature, the constant conductance model is inadequate, even for mild (20\%) step-power decreases. Second, we have seen that, although approximate analytical solutions to the case of temperature-dependant conductance can be found, in general, numerical solutions are more accurate. Third, we have seen that a timestep size of $0.1 \mathrm{sec}$ seems optimum for these numerical solutions. Lastly, we have shown that a log plot $\left[\ln \left(T_{N}-a\right)\right]$ of these calculations is preferable, since these plots have attributes (steepness and curvature) that are both independent of the thermocouple time constant and characteristic of a particular resistance-versus-power behavior.

These principles all apply to distributed parameter solutions, to which we turn next. The advantage in distributed parameter solutions is that they permit a partition of total resistance into fuel and gap resistances.

\section{OISTRIBUTED PARAMETER SOLUTIONS}

The so-called distributed parameter model of the fuel rod is a specialization of the general heat transfer equation. The general heat transfer equation is:

$$
p c \frac{\partial T(r, t)}{\partial t}=\nabla(K \nabla T)+Q(r, t)
$$


where $\rho=$ density

$C=$ specific heat capacity

$K=$ conductivity

$\mathrm{Q}=$ heat generation rate

The specialization to the fuel rod is made usually in the radial direction only, and consists of the following coupled equations:

for the fuel;

$$
\frac{1}{\alpha_{F}} \frac{\partial T_{f}}{\partial t}=\frac{\partial^{2} T_{f}}{\partial r^{2}}+\frac{1}{r} \frac{\partial T_{f}}{\partial r}+\epsilon_{F}\left(\frac{\partial T_{F}}{\partial r}\right)^{2}+\frac{Q(r, t)}{K_{f}}
$$

and for the cladding;

$$
\frac{1}{\alpha_{c}} \frac{\partial T_{c}}{\partial t}=\frac{\partial^{2} T_{c}}{\partial r^{2}}+\frac{1}{r} \frac{T_{c}}{r}+\beta_{c}\left(\frac{\partial T_{c}}{\partial r}\right)^{2}
$$

where

$$
\beta=\frac{1}{k}\left(\frac{d K}{d T}\right), \quad \alpha=\frac{K}{\rho C}
$$

The boundary conditions for the above set are:

$$
\begin{aligned}
& \frac{\partial T_{F}}{\partial r}=0, r=r_{\text {inner }} \\
& \left.K_{F} \frac{\partial T_{F}}{\partial r}\right|_{r_{F S}}=\left.K_{C} \frac{\partial T_{C}}{\partial r}\right|_{C S} \\
& {\left[T_{F}\left(r_{F s}, t\right)-T_{C}\left(r_{C S}, t\right)\right]_{\text {gap }}=K_{F} \frac{\partial T_{F}}{\partial r} \mid r_{F s}}
\end{aligned}
$$




$$
-\left.K_{C} \cdot \frac{\partial T_{c}}{\partial r}\right|_{r_{C O}}=h_{w}\left(T_{C}\left(r_{C O}, t\right)-T_{c o 0 l}\right)
$$

where $r_{F s}, r_{c s}$, and $r_{c o}$ are fuel radius and cladding inner and outer radii. Appropriate initial conditions must also be applied.

We will first consider the constant-parameter model, not because it is applicable to fuel rods in general but because, from simple analytical derivation, we can see the effect of varying resistance partition upon transient temperature predictions.

\section{Constant-Parameter Model (Cladding Excluded)}

Consider the fuel to be characterized by an effective conductivity, $\mathrm{K}$; a fuel surface-to-coolant conductance, $\mathrm{H}$; and volume heat capacity, C $\left(\right.$ joules $\left./ \mathrm{m}^{3}\right)$. The cladding is ignored except as a source of resistance. We also take $Q(r, t)=Q(t)$, and $r_{\text {inner }}=0$. Then Equations 20 and 21 reduce to:

$$
\frac{1}{\alpha_{F}} \frac{\partial T_{F}}{\partial t}=\frac{\partial^{2} T_{F}}{\partial r^{2}}+\frac{1}{r} \frac{\partial T_{F}}{\partial r}+\frac{Q}{K_{F}}
$$

with boundary conditions

$$
\begin{aligned}
& \frac{\partial_{F}}{\partial r}=0 \text { at } r=0 \\
& \left.K_{F} \frac{\partial T_{F}}{\partial r}\right|_{r_{F S}}=H\left[T\left(r_{F S}, t\right)-T_{C 001}\right]
\end{aligned}
$$

The initial condition for Equation 26 is taken to be $T(r, 0)=T_{0}(r)$ where $T_{0}$ is the solution to 


$$
0=\frac{\partial^{2} T_{0}}{\partial r^{2}}+\frac{1}{r} \frac{\partial T_{a}}{\partial r}+\frac{Q_{0}}{K}
$$

with boundary conditions

$$
\begin{aligned}
& \left.\frac{\partial T_{0}}{\partial r}\right|_{r=0}=0 \\
& -\left.K_{F} \frac{\partial T_{0}}{\partial r}\right|_{r_{F s}}=H_{0}\left(T\left(r_{F s}\right)-T_{C O O I}\right)
\end{aligned}
$$

This solution is:

$$
T_{0}=\frac{Q_{0}}{4 K_{F}}\left(r_{F s}^{2}-r^{2}\right)+\frac{Q_{0} r_{F S}}{2 H}+T_{C 001}
$$

These equations can be solved analytically in a variety of ways. For a step drop, $Q(t)=Q_{0}-\Delta Q, t>0$. The solution for the temperature response is:

$$
\begin{aligned}
T_{F}(r, t) & =\frac{Q_{0}-\Delta Q}{4 K_{F}}\left[r_{F s}^{2}-r^{2}+\frac{r_{F S}}{2 h}\right] \\
& +\frac{2 h \Delta Q}{r_{F s} K_{F}} \sum_{n=1}^{n}\left(\frac{J_{0}\left(X_{n} r\right) e^{-\alpha_{F} X_{n}{ }^{2} t}}{X_{n}{ }^{2}\left(X_{n}{ }^{2}+h^{2}\right) J_{0}\left(X_{n} r_{F s}\right)}\right)+T_{\text {cool }}
\end{aligned}
$$


where $\alpha_{F}=K / C$

$h=H / K$

Jo, $J_{1}$, are Bessel functions of zero and first order, and

$x_{n}$ are roots for $X J j_{1}\left({X r_{F s}}_{F}\right)=h$ Jo $\left({X r_{F s}}\right)$

If we just consider the centerline temperature and only consider times longer than four seconds so that the first term in the infinite series dominates, then we have:

$$
\begin{aligned}
T(0, t)-T_{C 001} & =\frac{\left(Q_{0}-\Delta Q r_{F s}^{2}\right)}{4 K}\left[1+\frac{2}{r_{F s} h}\right] \\
& +\frac{2 h \Delta Q}{r_{F s} K}\left(\frac{e^{-\alpha_{F} x_{1}{ }^{2} t}}{x_{1}^{2}\left(x_{1}{ }^{2}+h^{2}\right) J_{0}\left(x_{1} r_{F s}\right)}\right)
\end{aligned}
$$

From the steady-state initial condition we have

$$
T_{0}{ }^{(0)}-T_{C O 01}=\frac{Q_{0} r_{F s}^{2}}{4 K}\left[1+\frac{2}{r_{F s}^{h}}\right]
$$

Dividing both sides of Equation 30 by $\mathrm{T}_{0}(0)-\mathrm{T}_{\mathrm{CoO}}$, we have:

$$
T_{N}=\frac{Q_{0}-\Delta Q}{Q_{0}}+A e^{-\alpha x_{1}{ }^{2} t}
$$

where $A$ is a constant.

Defining "a" as $\left(Q_{0}-\Delta Q\right) / Q_{0}$, we have:

$$
T_{N}-a=A e^{-\alpha x_{1}{ }^{2} t}
$$


or

$$
-\ln \left(T_{N}-a\right)=-\ln A+\alpha x_{1}^{2} t
$$

Plots of $-\ln \left(T_{N}-a\right)$ should become straight lines with slopes $a X_{1}$.

Now various choices for $K$ and $H$ can be made, all of which yield the same centerline temperature. But each choice will result in a different value for $h=H / K$, which changes the characteristic Equation 34 and yields a different value for $x_{1}$ and, hence, for slope.

Using the values in Table 1, we can assess the effect of variation in resistance partition upon the slope of $-\ln \left(T_{N}-a\right)$ versus time. Pairs of $H$ and $\mathrm{K}$ values can be selected by solving Equation 32 for $\mathrm{H}$ in terms of $\mathrm{K}$ :

$$
H=\left[T_{0}(0)-T_{\cos 1}-\frac{Q_{0} F_{S}^{2}}{4 K}\right]^{-1} \frac{\left(Q_{0} r_{F s}\right)}{2}
$$

For $r_{F s}=0.00546 \mathrm{~m}$, we have the partitions of resistance shown in Table 2, for various choices of $K$. Table 3 summarizes the calculational steps involved in translating these portions to slopes of $-\ln \left(T_{N}-a\right)$. The corresponding calculated curves are presented in Figure 6 for reference.

Note that the difference in slopes is not great. If we seek to resolve differences in effective conductivity on the order of say, $5 \%$ relative, we will have to have very accurate solutions that take into account even minor effects, such as the effect of flux depression and energy storage in the cladding.

One can extend analytical solutions of the distributed (constant) parameter model to include the cladding, a central hole, and radially dependent heat generation; however, all of these solutions suffer the same flaw we noted for the constant lumped-parameter solution: the asymptotic value is, in general, incorrect. Approximate analytical solutions can be derived for 
TABLE 2. Possible Partitions of Resistance for the Data in Table 1

\begin{tabular}{|c|c|c|c|c|}
\hline \multirow{2}{*}{$\begin{array}{l}\text { Assigned } \\
\text { Fuel Conductivity } \\
\mathrm{W} / \mathrm{m}-\mathrm{K}\end{array}$} & \multicolumn{2}{|c|}{$\begin{array}{l}\text { Calculated Surface Conductance } \\
\mathrm{KW} / \mathrm{m}^{2}-\mathrm{K}\end{array}$} & \multicolumn{2}{|c|}{$\begin{array}{c}\text { Fraction of Resistance in } \\
\text { the Fuel, \& }\end{array}$} \\
\hline & High Resist. Rod & Low Resist. Rod & High Resist. Rod & Low Resist. Rod \\
\hline 2.5 & 5.093 & -- & 84.1 & -- \\
\hline 3.0 & 2.643 & -- & 70.6 & -- \\
\hline 3.5 & 1.967 & -- & 60.5 & -- \\
\hline 4.0 & 1.657 & 18.317 & 52.9 & 92.6 \\
\hline 4.5 & -- & 7.667 & -- & 82.3 \\
\hline 5.0 & -- & 5.223 & -- & 74.1 \\
\hline
\end{tabular}

TABLE 3. Slopes of $\ln \left(T_{N}-a\right)$

\begin{tabular}{|c|c|c|c|c|}
\hline \multirow{2}{*}{$\begin{array}{c}\text { Assigned } \\
\text { Conductivity } \\
\end{array}$} & \multicolumn{2}{|c|}{$\begin{array}{c}\text { Roots of Characteristic Equation } \\
\qquad x, m^{-1}\end{array}$} & \multicolumn{2}{|c|}{$\begin{array}{c}\text { Slope of } \ln \left(T_{N}-a\right) \\
\sec -1\end{array}$} \\
\hline & High Resist. Rod & Low Resist. Rod & High Resist. Rod & Low Resist. Rod \\
\hline 2.5 & 4.0306 & -- & 0.13702 & -- \\
\hline 3.0 & 3.6198 & -- & 0.12599 & -- \\
\hline 3.5 & 3.2941 & -- & 0.12173 & - \\
\hline 4.0 & 3.0350 & 4.2324 & 0.11809 & 0.22966 \\
\hline 4.5 & -- & 3.9636 & -- & 0.22659 \\
\hline 5.0 & -- & 3.7251 & -- & 0.22238 \\
\hline
\end{tabular}




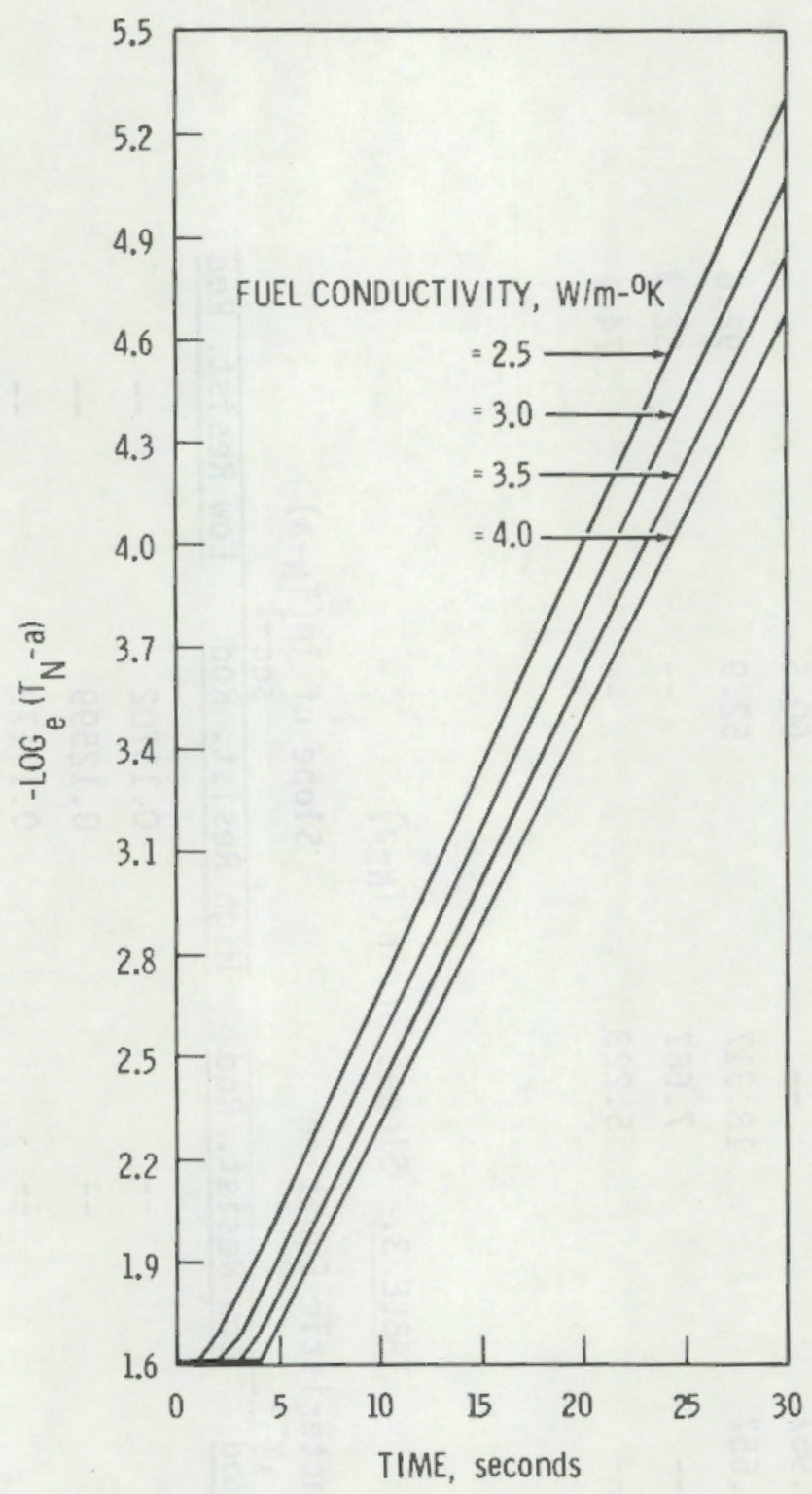

FIGURE 6a. Effects of Different Resistant Partitions in the High-Resistance Rod 


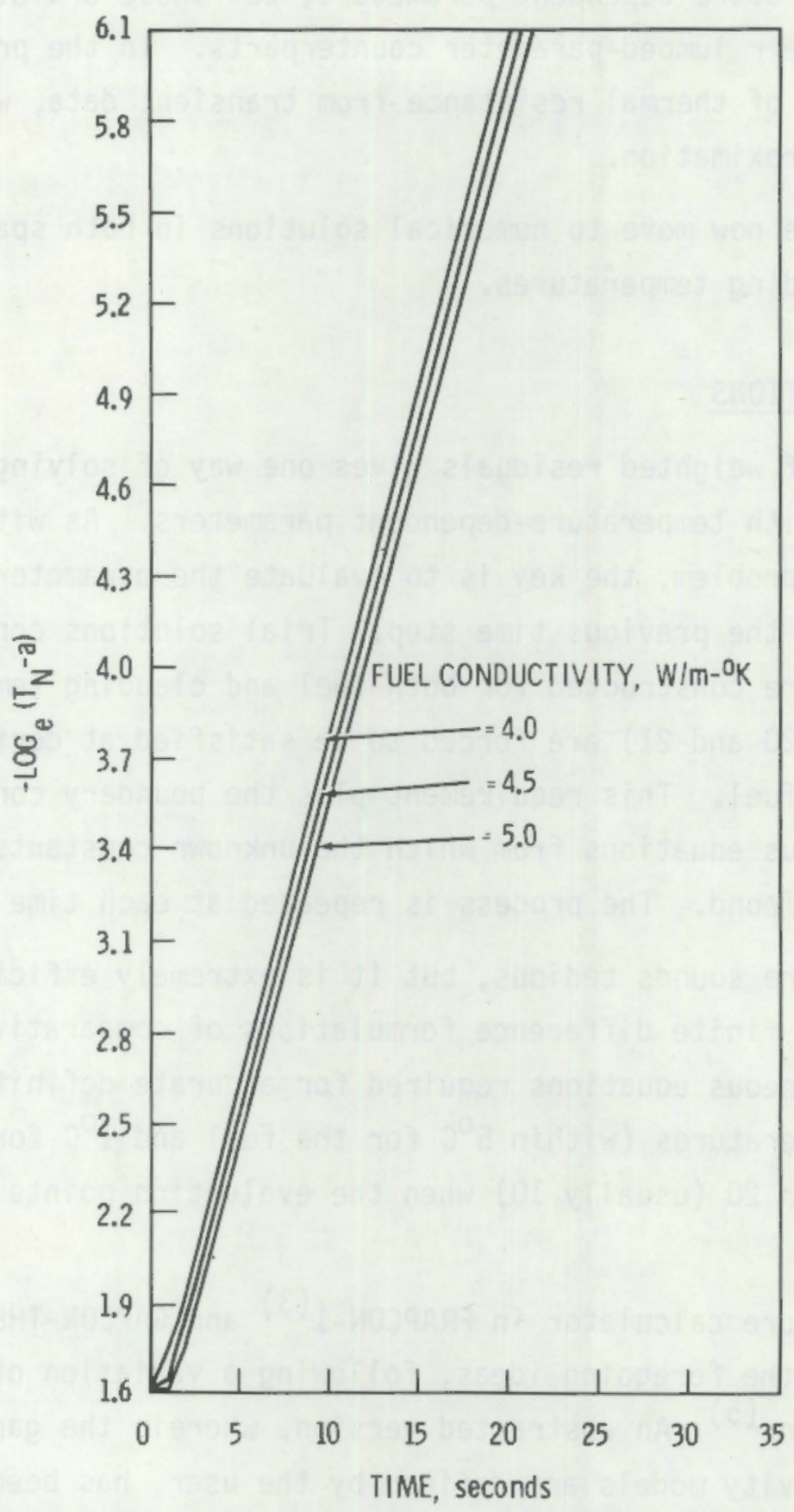

FIGURE 6b. Effects of Different Resistance Partitions in the Low-Resistance Rod 
the case of temperature-dependent parameters, but these are at least as approximate as their lumped-parameter counterparts. In the process of assessing the partition of thermal resistance from transient data, we cannot tolerate that level of approximation.

Therefore, we now move to numerical solutions in both space and time for the fuel and cladding temperatures.

\section{NUMERICAL CALCULATIONS}

The method of weighted residuals gives one way of solving the distributed parameter model with temperature-dependent parameters. As with the nonlinear lumped parameter problem, the key is to evaluate the parameters in terms of temperatures from the previous time step. Trial solutions consisting of power series of radii are constructed for both fuel and cladding temperatures. The model equations (20 and 21 ) are forced to be satisfied at certain points in the cladding and fuel. This requirement plus the boundary conditions form a set of simultaneous equations from which the unknown constants in the series expansion may be found. The process is repeated at each time step.

This procedure sounds tedious, but it is extremely efficient compared to finite element or finite difference formulations of comparative accuracy. The number of simultaneous equations required for accurate definition of the fuel and cladding temperatures (within $5^{\circ} \mathrm{C}$ for the fuel and $1^{\circ} \mathrm{C}$ for the cladding) is less than 20 (usually 10) when the evaluation points are judiciously chosen.

The temperature calculator in FRAPCON-1 ${ }^{(3)}$ and GAPCON-THERMAL-3 ${ }^{(4)}$ was constructed from the foregoing ideas, following a variation of the method proposed by Finlayson. (5) An abstracted version, wherein the gap conductance and fuel conductivity models are defined by the user, has been constructed as the small code MWRAM. A description and listing of MWRAM is given in the appendix. In this section we examine the results of some MwRAM calculations that reveal the effect of using different models that are all constrained to match the same steady-state temperature-versus-power data. Some twodimensional cases from the finite-difference program TRUMP(6) will also be examined. 


\section{Constant Conductance Versus Temperature Dependent Conductance}

There are two extreme views called "Model 1 and Model 2," regarding the behavior of the fuel during step-power decreases. They are described as follows:

Model 1: In this model there are no fuel cracks so there is no impediment to radial heat flow caused by fuel cracks. The fuel conductivity is taken to be of the Lyons form. ${ }^{(7)}$ The changes in gap conductance with power are assumed to be dominated by the thermal expansion of the fuel and cladding, which, in turn is related to their respective temperatures. Since over the range of the power decrease, the change in temperature with power is fairly uniform across the entire rod, a linear dependence of gap conductance on the volume-averaged fuel temperature will be assumed. (a)

Mode1 2: In this model the fuel has many small and randomly oriented cracks, which impede the radial flow of heat. The latter can be accounted for by applying a temperature-dependent multiplier to the fuel thermal conductivity. The fuel is envisioned to be fully relocated to the cladding and well cracked so that over the range of the power decrease there is little change in the gap conductance. The change in resistance with power is presumed to be totally due to the change in effective conductivity, dominated by the crack factor. The multiplier on conductivity is assumed to be linearly dependent on the volume-averaged fuel temperature (presuming this to be a guide to pellet-cladding mechanical interaction that could close the fuel cracks when power is increased).

The values in Table 1 can be used to make Model 1- and Model 2-type calculations. For a Model 1 calculation, the fuel surface temperature $\left(T_{F s}\right)$ is estimated from the relation:

(a) Linear dependence of conductance on fuel volume averaged temperature is evident in FRAPCON and GAPCON runs. 


$$
\int_{T_{F s}}^{T} \begin{gathered}
\text { center } \\
\text { cit }
\end{gathered}=\frac{Q r_{F s}{ }^{2}}{4}
$$

where $K=\frac{A}{T+B}+C T^{3}$

The fuel surface temperature can be used to calculate a conductance, $h_{\text {gap }}$ :

$$
h_{g a p}=\frac{Q r_{F s}{ }^{2}}{2 r_{F s}\left(T_{F s}{ }^{-T} C I\right)}=\frac{Q r_{F s}}{2\left(T_{F s}{ }^{-T_{C I}}\right)}
$$

where $T_{C I}$ is the cladding inner surface temperature.

As explained in the appendix, ${ }^{T} C I$ is calculated in a manner similar to $T_{F s}$. The procedure is repeated for both the initial and final steady states, and gap conductance as a linear function of volume-averaged temperature is thus determined.

A Model 2 calculation is formed along different lines. The conductance is arbitrarily chosen and fixed throughout the transient. Any physically reasonable value may be used. The surface temperatures are found by solving Equation 39 for both the initial and final steady states. Then, for both power levels, the multiplier $k_{\text {mult }}$ is found such that: 


$$
K_{\text {mult }} \int_{T_{F s}}^{T_{\text {centeg }}{ }_{\text {L } a b}}=\frac{Q r_{F s}{ }^{2}}{4}
$$

Then $K_{\text {mult }}$ can be found as a linear function of volume-averaged temperature.

Now the same set of initial and final steady-state values can be used to "tune" both calculations. The resistance-versus-power predictions of each model will then be nearly identical over the range of the power drop. This is shown in Figure 7, which includes the data from Table 4.

The predicted response to a $20 \%$ step-power decrease, using both models in MWRAM, is shown in Figure 8 . Note that although the resistance-versus-power predictions are almost identical, the transient predictions definitely are not.

Even if we use the same model, we find again that different partitions of resistance will result in different predicted transient response. For example when we inserted a series of arbitrarily chosen conductances in Model 2 and adjusted the fuel conductivity to match the data (Table 1), the curves shown in Figure 9 were generated. The chosen conductances and corresponding conductivity changes are shown in Table 5. Note the divergence which occurs in the predicted slope of $\ln \left(T_{N}-a\right)$ versus time.

TABLE 4. Input Parameters for Comparative MURAM Calculations (High Resistance Rod)(a)

\begin{tabular}{|c|c|c|}
\hline I ten & Value & Units \\
\hline Initial/Final Power & $27.2 / 21.7$ & $\mathrm{~kW} / \mathrm{m}$ \\
\hline Initial/Final Temp. & $1331 / 1216$ & ${ }^{\circ} \mathrm{C}$ \\
\hline Fuel ID/OD & $0.00175 / 0.01090$ & $m$ \\
\hline Clad ID/OD & $0.01097 / 0.0128$ & m \\
\hline
\end{tabular}

(a) For fixed parameters in MWRAM see Appendix A 


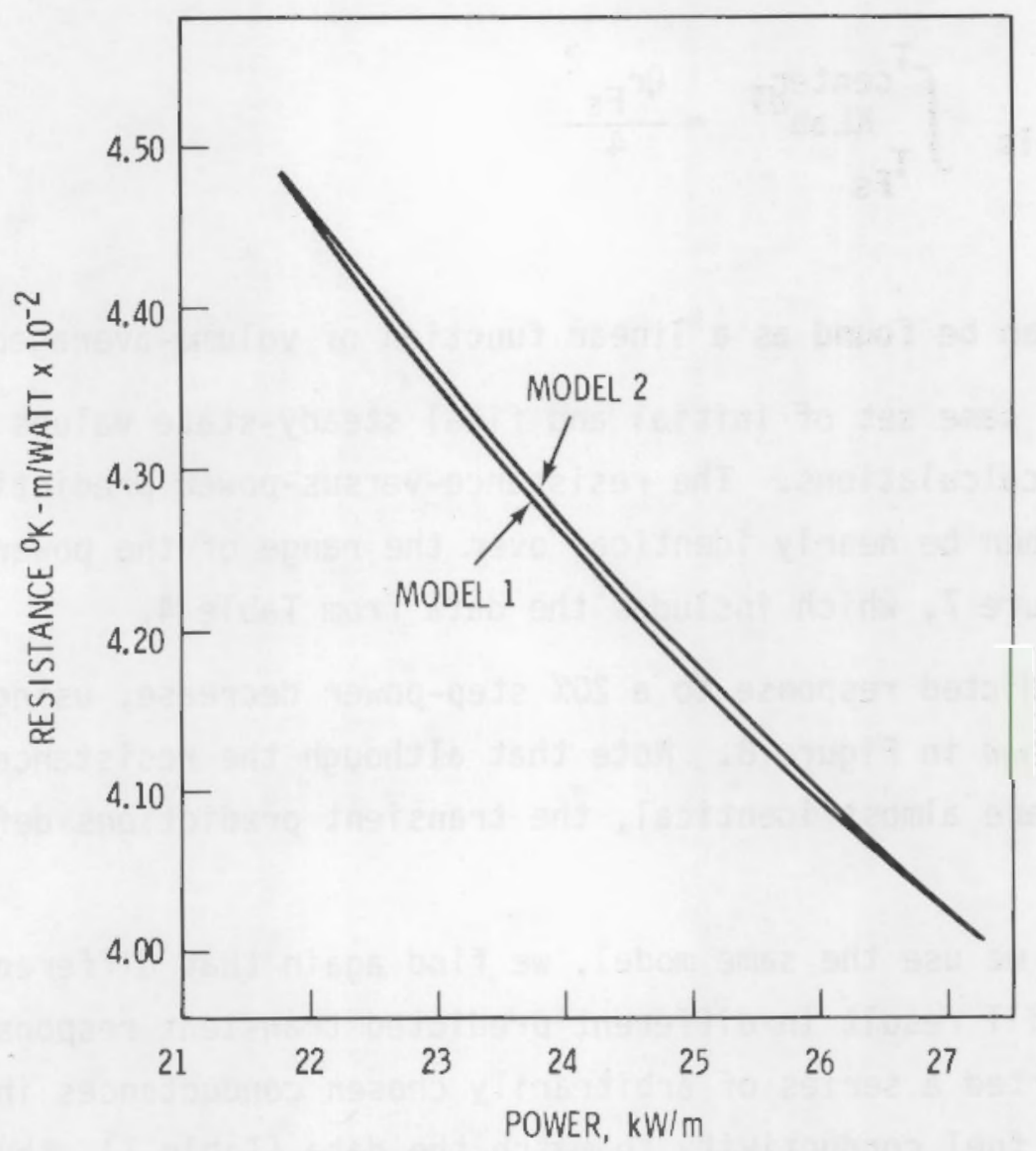

FIGURE 7. Resistance versus Power for the High-Resistance Rod, as Deduced from Initial/Final Steady-State Conditions 


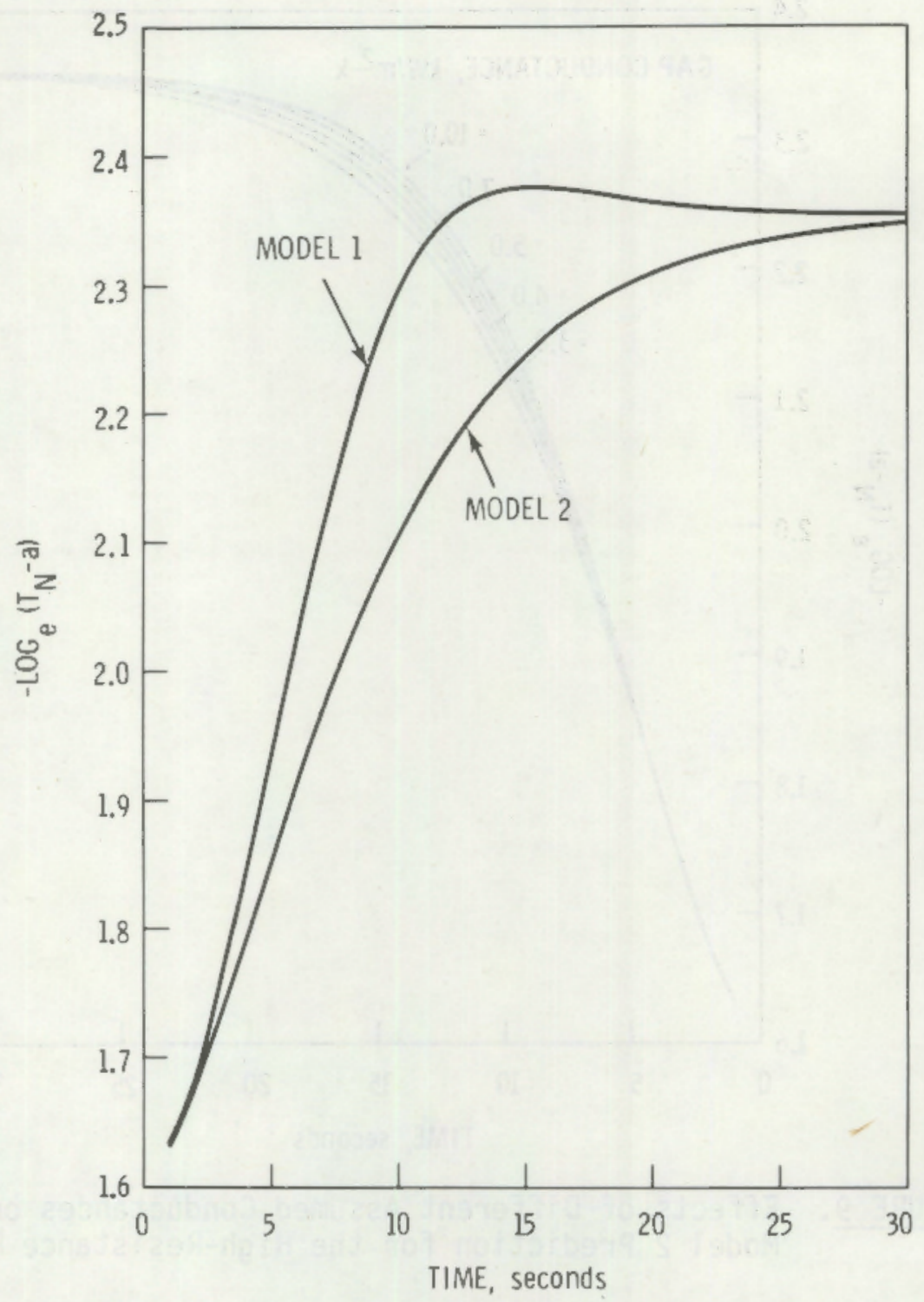

FIGURE 8. Model 1 and 2 Predictions for a $20 \%$ Power Decrease in the High-Resistance Rod 


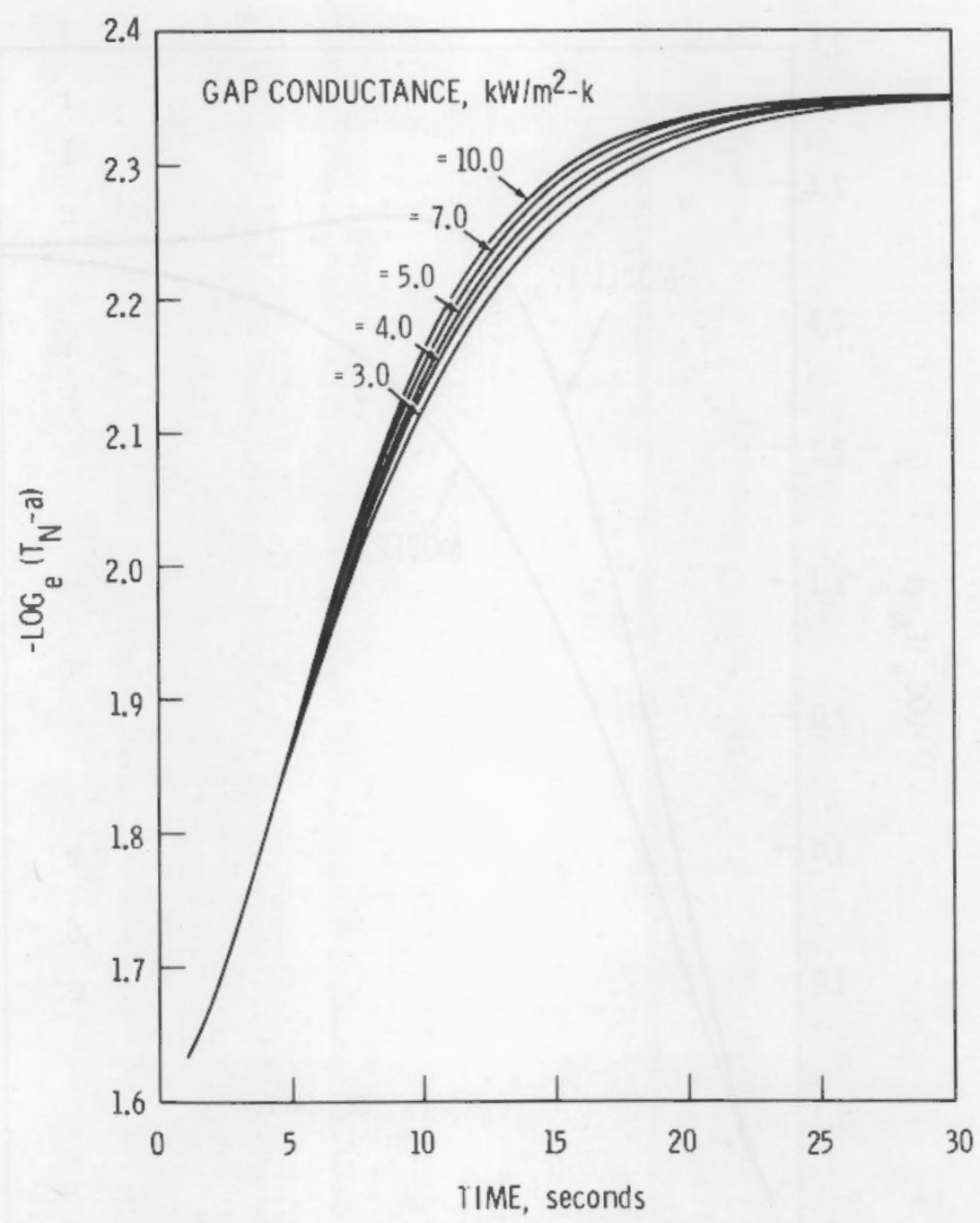

FIGURE 9. Effects of Different Assumed Conductances on the Model 2 Prediction for the High-Resistance Rod 


\section{TABLE 5. Results of Various Choices of Gap Conductances}

(Model 2, High-Resistance Rod)

\begin{tabular}{|c|c|c|}
\hline $\begin{array}{l}\text { Gap Conductance, } \\
\mathrm{kW} / \mathrm{m}-{ }_{\mathrm{C}}\end{array}$ & $\begin{array}{l}\text { Initial } \\
\text { Conductivity } \\
\text { Multiplier } \\
\end{array}$ & $\begin{array}{c}\text { Initial Fraction, } \\
\text { of Resistance in } \\
\text { Fuel, } \%\end{array}$ \\
\hline 2.5283 & 1.00 & 67 \\
\hline 3.00 & 0.9173 & 71 \\
\hline 4.00 & 0.8213 & 77 \\
\hline 5.00 & 0.7701 & 81 \\
\hline 7.0 & 0.7166 & 85 \\
\hline 10.00 & 0.6794 & 88 \\
\hline
\end{tabular}

\section{TWO-DIMENSIONAL CALCULATIONS}

The results of the MWRAM calculations look promising for assessing azimuthally averaged resistance partitions in comparison to data. But it is well to check whether two-dimensional effects, such as flux tilt or azimuthally varying conductance, could bias the centerline temperature versus time so much as to invalidate the use of the one-dimensional model. In order to make some two-dimensional calculations, we used the general purpose finite difference heat transfer code TRUMP. (6)

The basic mesh used for all calculations is similar to the one shown in Figure 10. It consists of 20 radial rings ( 6 in the cladding) plus thirty $12^{\circ}$ azimuthal segments. Three cases were considered:

a) An eccentric pellet (with no flux depression or flux tilt) (100\% drop in power).

b) A concentric pellet with $40 \%$ edge-to-edge flux tilt but no flux depression (20\% drop in power).

c) A concentric pellet with parabolic flux depression (center-to-edge ratio $=1 / 1.3$ ) with $40 \%$ flux depression ( $20 \%$ drop in power).

In each case the mean gap (the hot gap) was 75 microns and filled with helium. Table 6 lists the other major parameters that were used. 


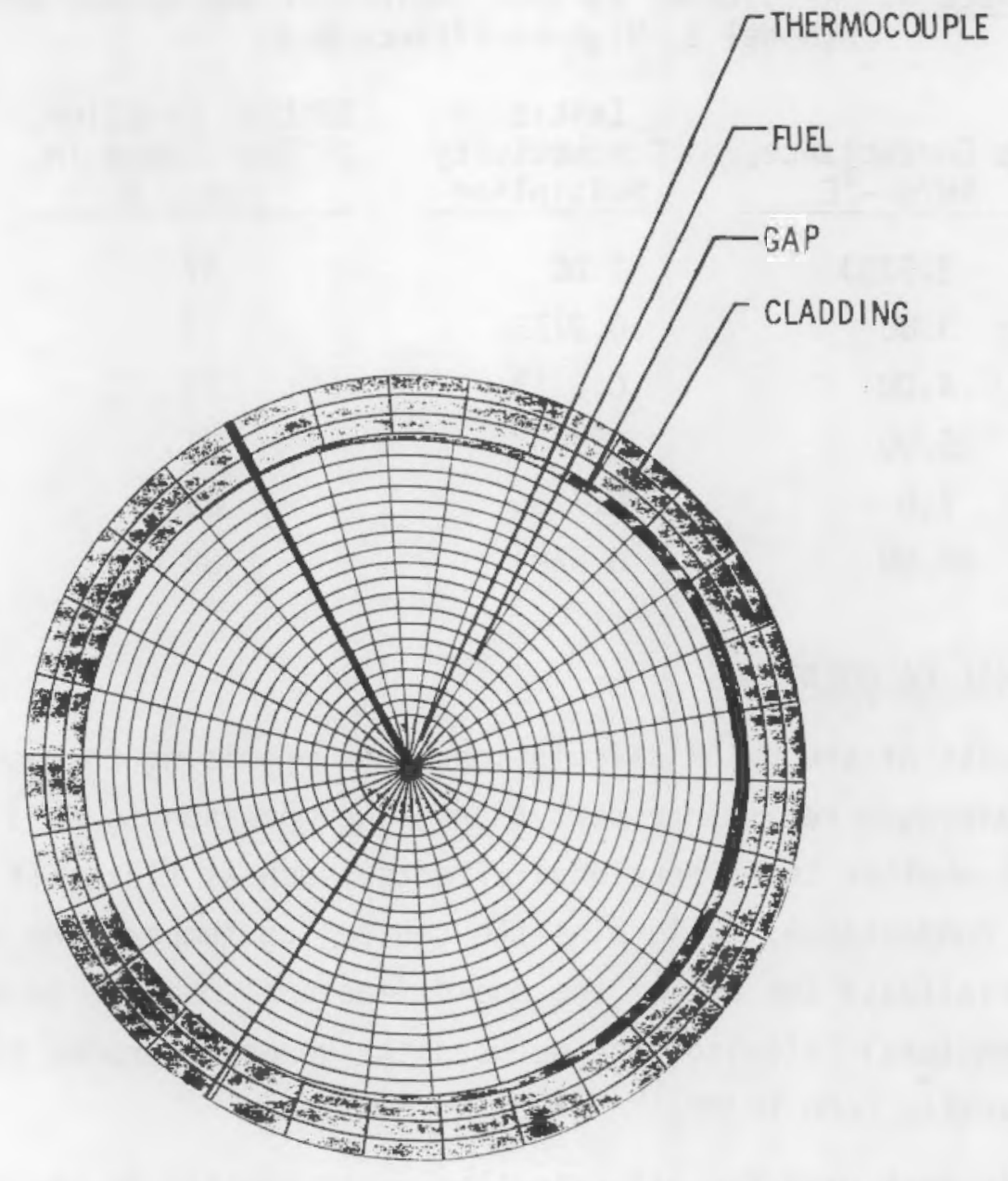

FIGURE 10. Two-Dimensional Mesh for TRUMP Calculations 
TABLE 6. Major Input Parameters for Two-Dimensional Comparative Cases

\begin{tabular}{|c|c|c|c|}
\hline Item & Value & Units & Reference \\
\hline Power & 27.42 & $\mathrm{~kW} / \mathrm{m}$ & -- \\
\hline Fuel Conductivity & Lyons Equation & $\mathrm{kW} / \mathrm{m}^{\circ} \mathrm{C}$ & (7) \\
\hline Fuel ID & 0.00175 & $m$ & -- \\
\hline Fuel 00 & 0.01068 & m & -- \\
\hline Clad ID & 0.01092 & m & -- \\
\hline$C 1$ ad $O D$ & 0.01282 & $m$ & -- \\
\hline $\begin{array}{l}\text { Cladding } \\
\text { Conductivity }\end{array}$ & $13-15$ & $W / m^{0} \mathrm{C}$ & (8) \\
\hline Fuel Heat Capacity & Equation of Bates & $\mathrm{j} / \mathrm{Kg}^{\circ} \mathrm{C}$ & (9) \\
\hline Fuel-Clad Mean Gap & 75 & microns & -- \\
\hline Gap Gas Conductivity & $\begin{array}{l}\text { Equation of Wilke } \\
\text { for pure Helium }\end{array}$ & $\mathrm{W} / \mathrm{m}^{\circ} \mathrm{C}$ & (10) \\
\hline $\begin{array}{l}\text { Center-to-edge flux } \\
\text { ratio(a) }\end{array}$ & 1.3 & -- & -- \\
\hline
\end{tabular}

(a) For cases involving flux depression

\section{Effect of Eccentricity on Mean Fuel Center Temperature}

We used the data in Table 6 to contrast the centerline temperature response of a concentric and an eccentric pellet to a $100 \%$ power decrease. In the eccentric case, the azimuthally averaged centerline temperature was calculated. The comparison of the two cases is shown in Figure 11, in terms of $\ln \left(T_{N}-a\right)$ versus time. The two results are nearly identical, with respect to slope, after about 4 seconds. This is instructive. It means that transient centerline temperature data cannot be used to distinguish the effects of pellet eccentricity, and any gap conductance information deduced from 


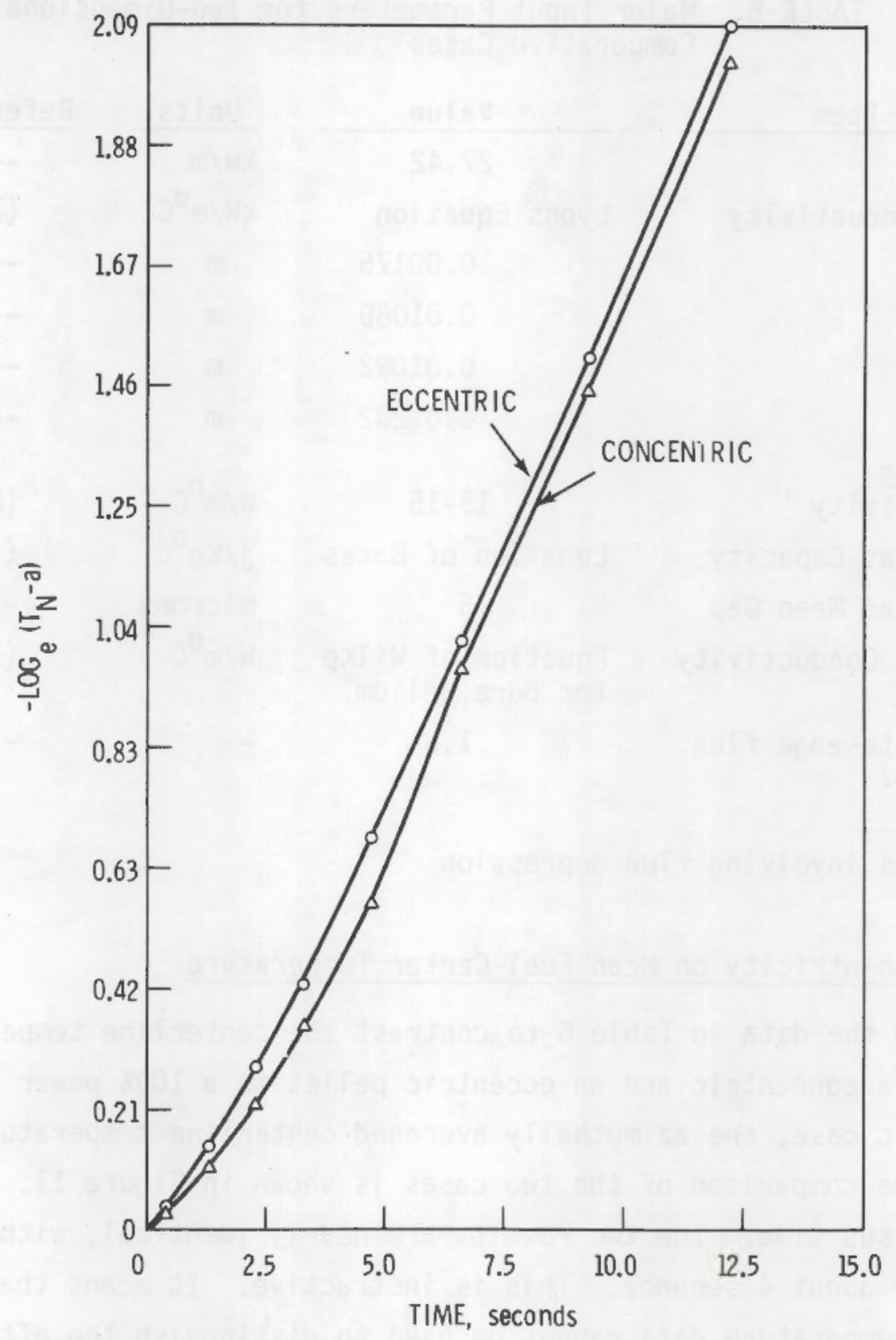

FIGURE 11. Response of Concentric and Eccentric Pellets to a 100\% Power Decrease (TRUMP Calculations) 
centerline transient temperatures must be understood as being azimuthally averaged, just as with steady-state centerline temperatures and associated gap conductances. On the other hand, a purely eccentric pellet is probably just as idealized as a purely concentric one; and random pellet fragmentation and relocation actually favors the concentric model over the eccentric one.

\section{The Effects of Planar Flux Tilt}

The next case considered is that of a concentric pellet subjected to a planar flux tilt of $40 \%$. The responses to a $20 \%$ power decrease, with and without the tilt, are shown in Figure 12. The two results are so close as to be indistinguishable. Again, the azimuthally averaged thermocouple temperature is plotted.

Adding flux depression to the flux tilt results in a skewed heat generation rate, as shown in Figure 13. However, the transient centerline response in this case is nearly identical to that for the same case with no flux tilt. This is shown in Figure 14.

These last two cases point out that the centerline transient temperature response is also incapable of detecting significant flux tilt. On the other hand, edge-to-edge tilts of greater than $10 \%$ across a single rod are rarely encountered, and these do not perturb the gap conductance or the fuel/cladding temperatures very greatly. The effect of the magnitude of the flux tilt on the extreme variations of temperature is shown in Table 7.

In conclusion, we have shown that transient centerline temperatures are insensitive to two-dimensional effects; however, those effects are expected to be small and random in real fuel rods. Transient centerline temperature data analysis will be discussed next; the results given apply in an azimuthally averaged sense. 


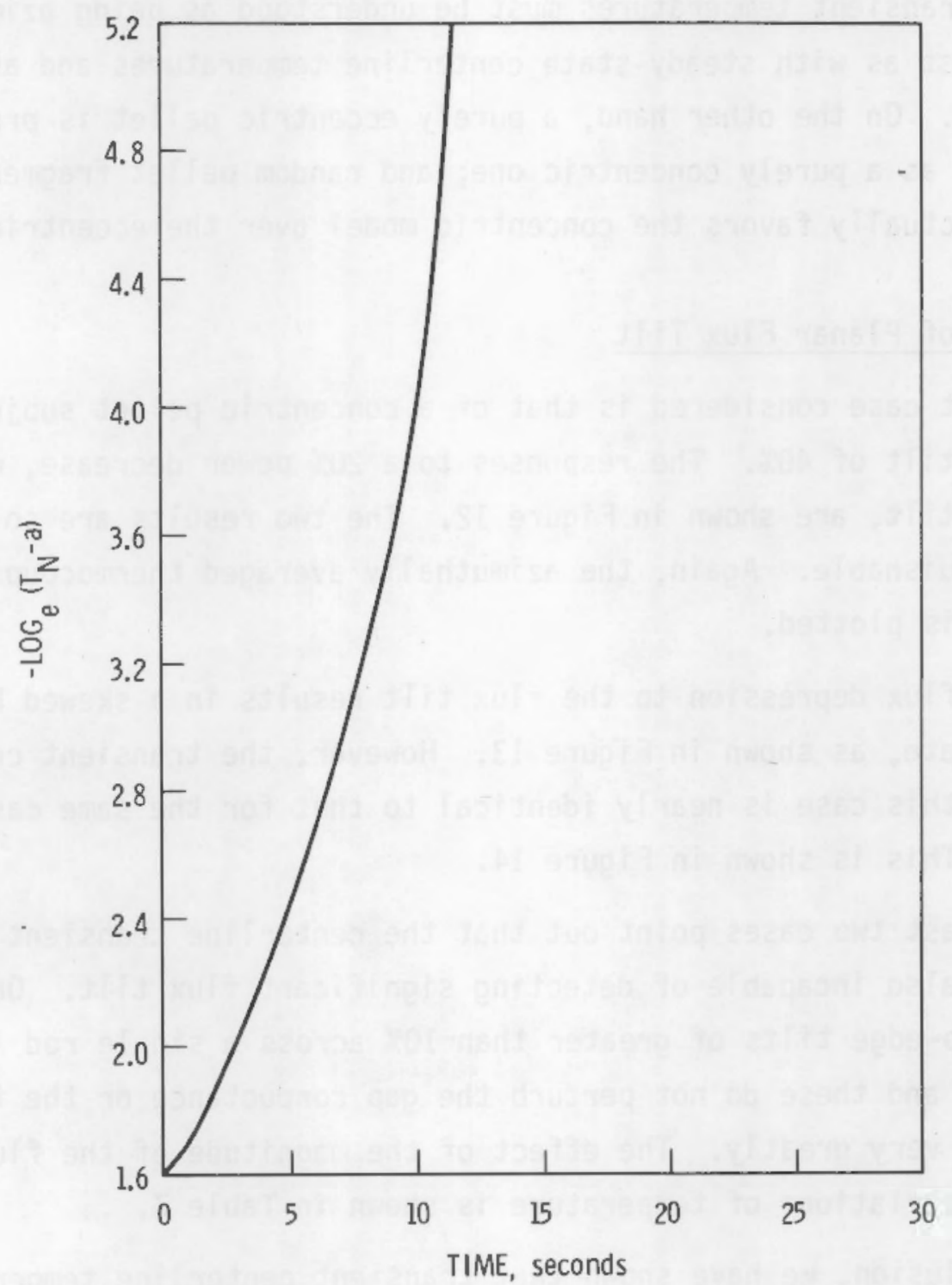

FIGURE 12. Calculated Response of a 3-mil Hot Gap Rod (to a $20 \%$ Power Decrease) With and Without a 40\% Flux Tilt 


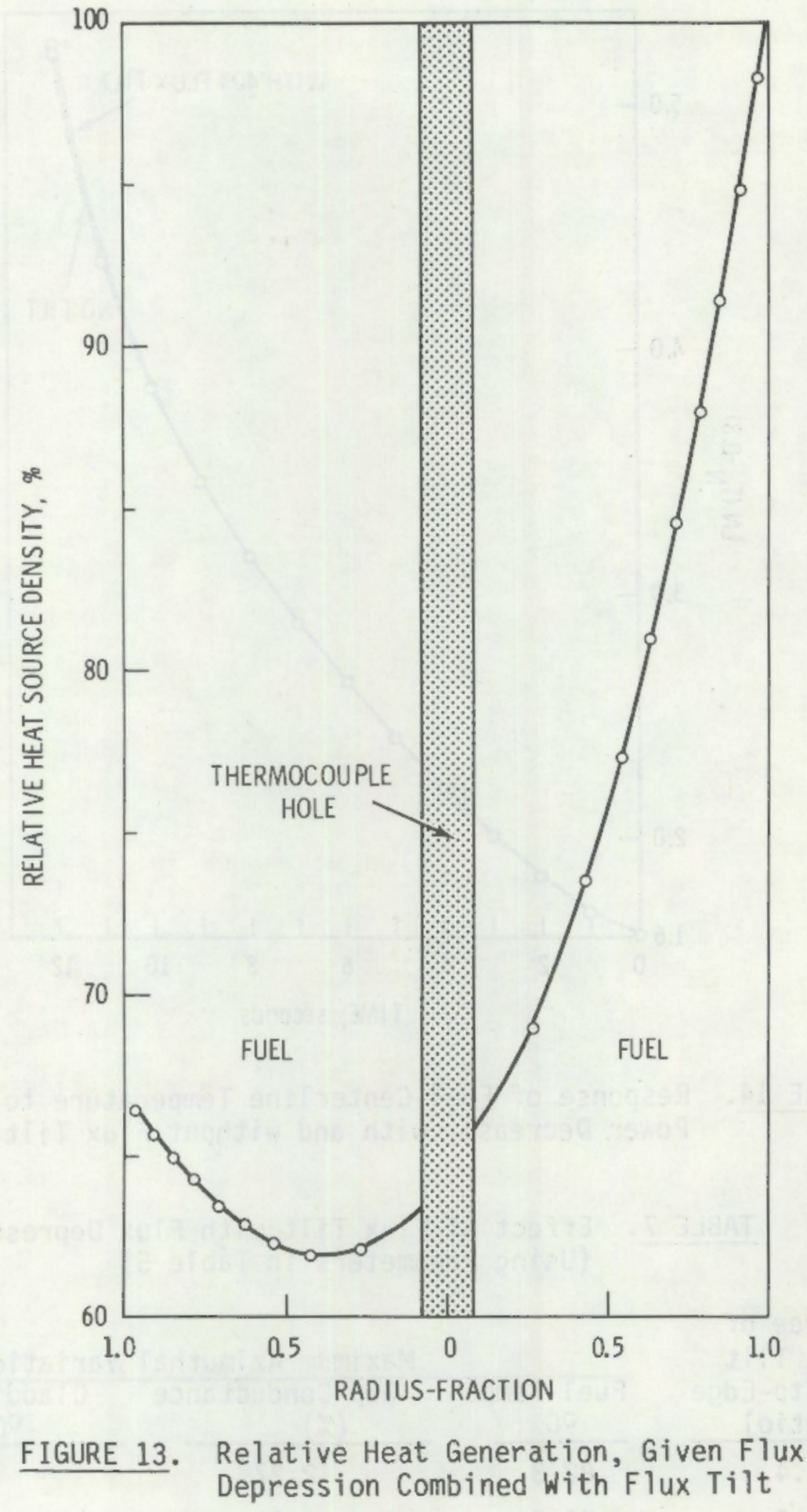




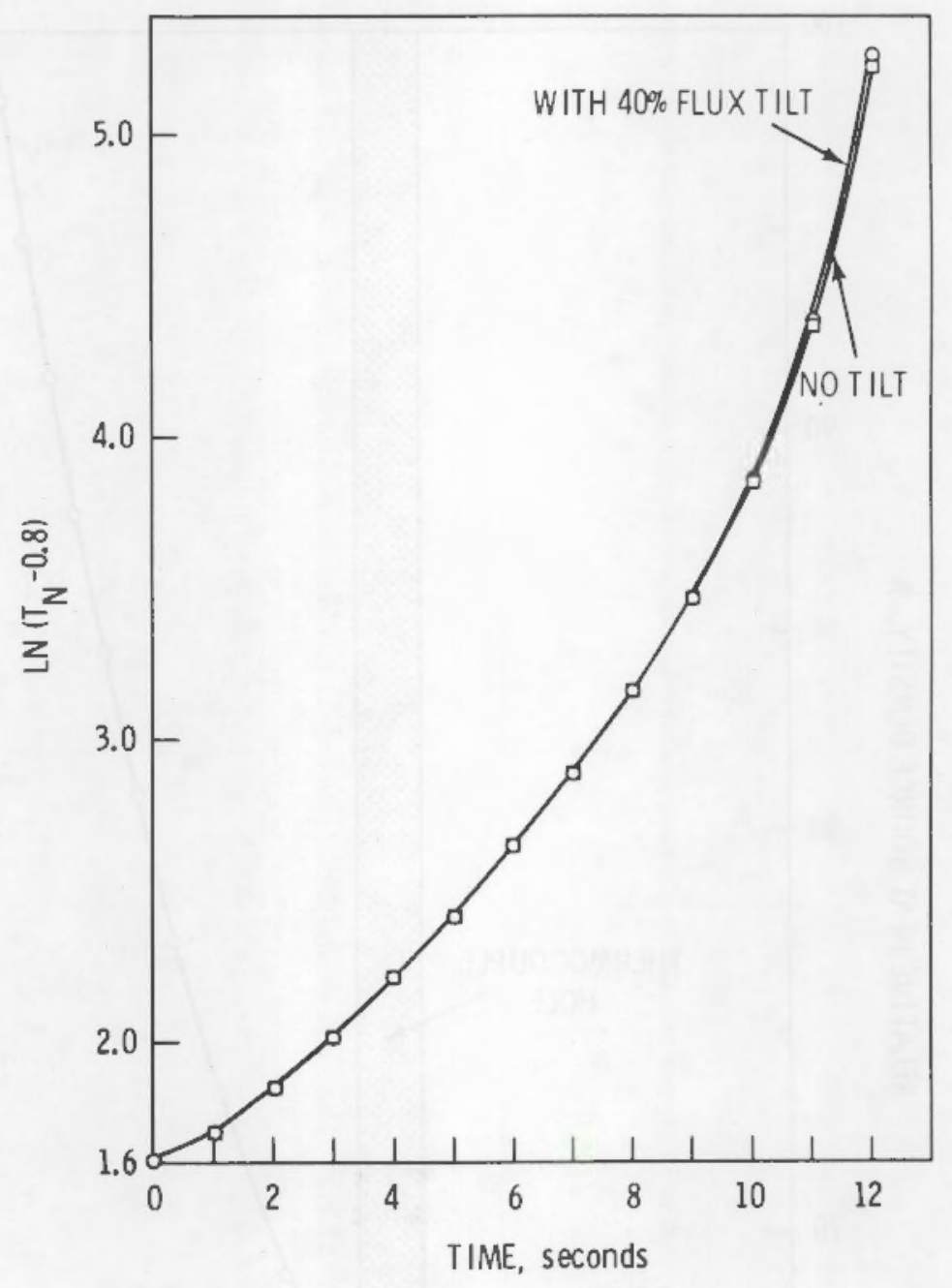

FIGURE 14. Response of Fuel Centerline Temperature to a Step 20\% Power Decrease, with and without Flux Tilt

TABLE 7. Effect of Flux Tilt with Flux Depression (Using Parameters in Table 5)

Degree of

Flux Tilt (Edge-to-Edge ratio)

1.4

1.2

1.1
Maximum Azimuthal Variations

\begin{tabular}{ccc}
\hline $\begin{array}{c}\text { Fuel Temp. } \\
{ }_{\text {OC }}\end{array}$ & $\begin{array}{c}\text { Gap Conductance } \\
(\%)\end{array}$ & $\begin{array}{c}\text { Cladding Temp. } \\
\text { OC }\end{array}$ \\
82.3 & 2.27 & 7.7 \\
41.2 & 1.13 & 3.8 \\
20.6 & 0.57 & 1.9
\end{tabular}




\subsection{SAMPLE DATA INTERPRETATION}

This section includes our procedure for the qualitative interpretation of the fuel thermocouple response to a step-power decrease. In summary, this procedure includes the following steps:

1. Establish initial/final steady-state conditions and compare the Models 1 and 2 against the transient thermocouple data(a). This almost always establishes Model 2 as the more appropriate of the two.

2. Do survey calculations (using Model 2) in which the gap conductance is varied from case to case.

3. From the results of the survey, establish a probable range for the conductance, and a corresponding range of fuel thermal conductivity degradation.

The data we will consider comes from the upper centerline thermocouple of Rod 6 , IFA-513(b). This rod had an initial fill gas composition of $77 \%$ He, $23 \% \mathrm{Xe}$. The fabricated diametral gap was $230 \mu \mathrm{m}$, the fuel inner and outer diameters were 0.001725 and $0.01068 \mathrm{~m}$, respectively and cladding inner and outer diameters were 0.01090 and $0.0128 \mathrm{~m}$, respectively. We will examine the behavior of the upper thermocouple of rod 6 during 20\% step-drop transients that occurred after 4,50 , and 80 days of irradiation. These transients are respectively designated runs 115,126 and 141 .

The resistance behavior at this thermocouple location is plotted as a function of burnup (over a 100-day time span) in Figure 15. The power levels are indicated on the figure, and so are the times at which transient data was taken. Note that the resistances throughout the burnup range are fairly constant.

Following the procedure above, we will first compare the data from all three transient runs to Models 1 and 2. Then we will compare the results of survey calculations (using a range of gap conductance values) to the data from all 3 runs.

(a) The models were described on page 31.

(b) The six-rod assembly, IFA-513, is an instrumented test fuel assembly in the Halden Reactor, Norway. It was designed for the Nuclear Regulatory Commission by Pacific Northwest Laboratory. 


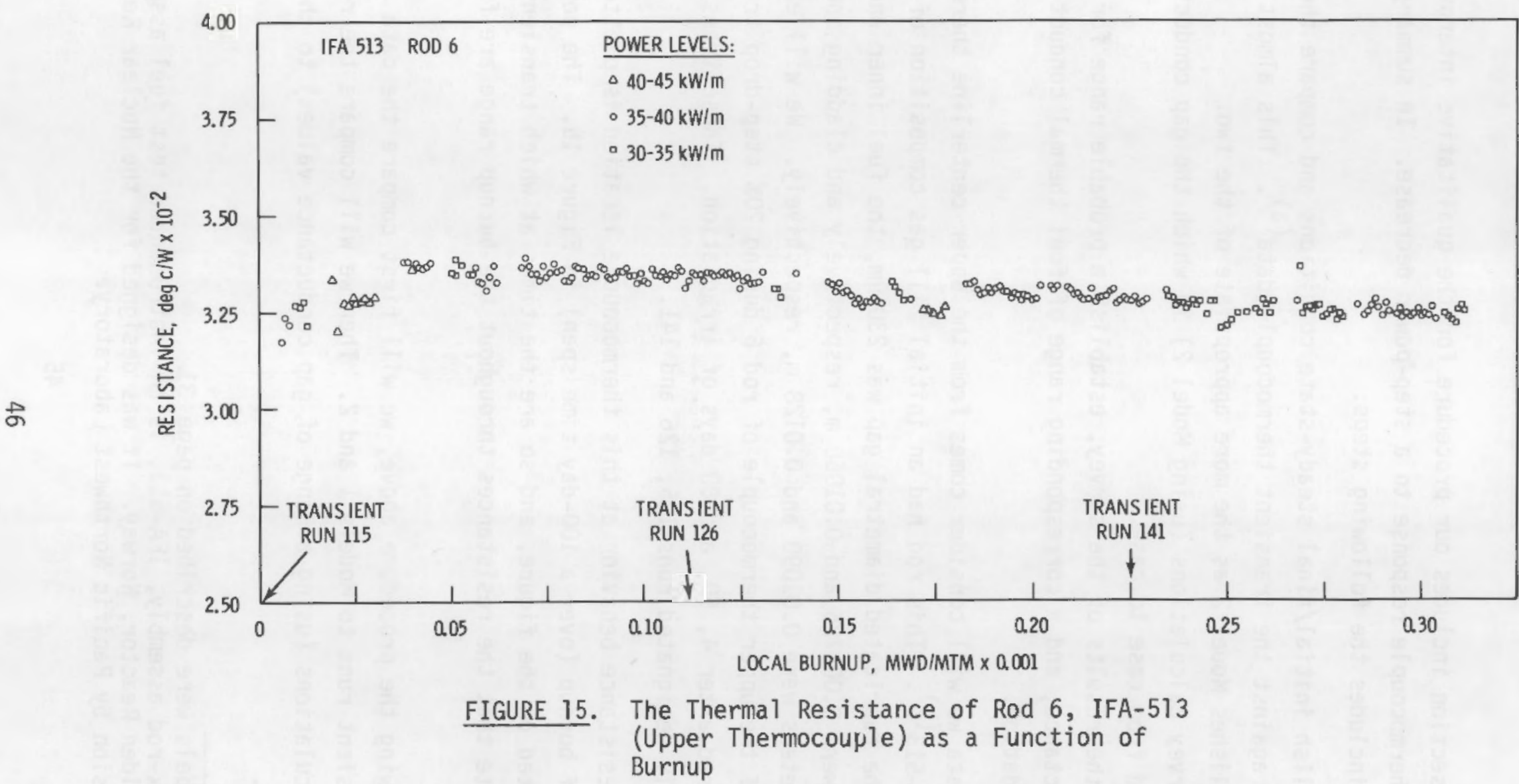


MODEL 1/MODEL 2 COMPARISONS

The first step in comparing Models 1 and 2 against transient data is to establish the initial and final steady-state conditions for the transient. That is, the initial/final powers that are associated with the initial/final temperatures must be determined. With present transient data coming from Halden, this is not straightforward, because the dedicated computer system (which records the transient data) only records the thermocouple readings and the output of the fast-reacting cobalt detectors. The output of slowerreacting vanadium neutron detectors (from which the local powers are determined), are only recorded on the steady-state test fuel data report (TFOR) system. The association of correct initial/final temperatures with powers thus requires some detective work and proceeds as follows:

a. The transient temperature and cobalt detector readings are plotted for a considerable period, both before and after the actual step drop, to ascertain the steady-state levels.

b. A temperature-versus-power curve is developed from the TFDR data available near the time of the power drop, so that the correct power can be associated with the initial/final steady-state temperatures.

c. As a cross check, the ratio of final/initial powers picked of the temperature-versus-power curve is compared to the ratio of initial/final cobalt detector readings.

The recorded temperatures from the rod 6 upper thermocouple and the corresponding cobalt neutron detector signal for the first transient (run 115) are shown in Figure 16. The initial and final steady-state levels are noted on the figure. In Figure 17 we show the temperature-versus-power curve for the thermocouple from a staircase rise to power imnediately preceeding Run 115. Note the powers that correspond to the steady-state temperatures. The ratios of initial/final powers and cobalt detector readings agree fairly well:

Cobalt detector readings: $\quad 65.7 / 80.8=0.813$

Associated powers:

$33.5 / 41.6=0.805$ 


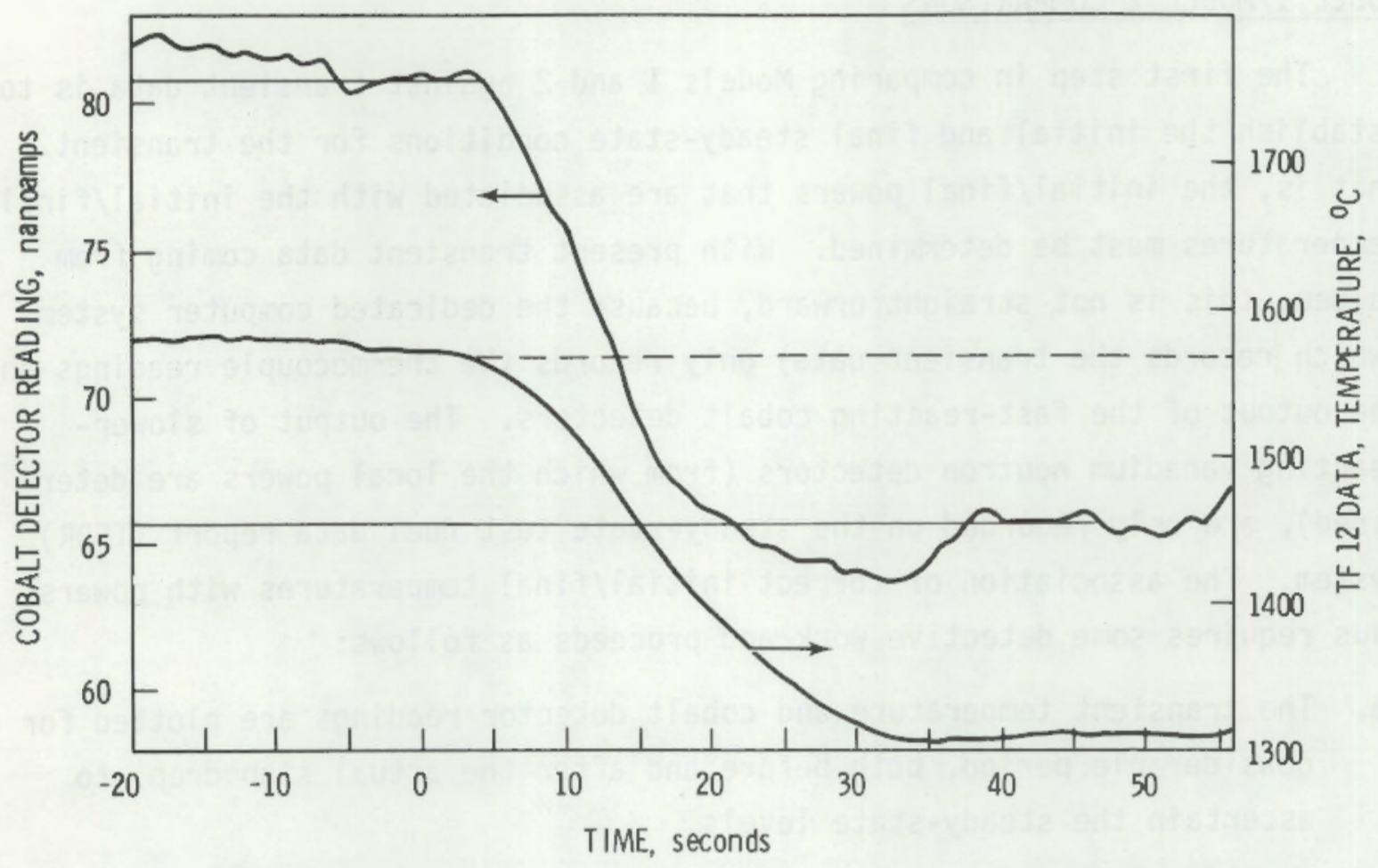

FIGURE 16. Unnormalized Temperature and Cobalt Detector Signal for Rod 6 (Upper Thermocouple) - Run 115

These initial/final conditions can now be input to MWRAM, which will output the associated gap conductances and crack factors. Table 8 includes the results of this procedure for TF 12 for all 3 runs. We see from the table that, whereas the resistances in all cases are very nearly the same, the gap conductances are not. Run 141 occurred at a lower initial power (and consequently lower fuel temperatures and higher fuel conductivity) than the other two transient runs. The conductance required to produce equivalent resistance

TABLE 8. Initial/Final Conditions and MWRAM Parameters for TF 12 (Rod 6 Upper Thermocouple)

\begin{tabular}{|c|c|c|c|c|c|}
\hline $\begin{array}{l}\text { Transient } \\
\text { Run Number } \\
\end{array}$ & $\begin{array}{l}\text { Initial/Final } \\
\text { Power, kW/m } \\
\end{array}$ & $\begin{array}{r}\text { Initial/Final } \\
\text { Temperature }\left({ }^{\circ} \mathrm{C}\right) \\
\end{array}$ & $\begin{array}{l}\text { Initial/Final } \\
\text { Resistance } \\
\text { (OC m/Watt) }\end{array}$ & $\begin{array}{r}\text { Initial/Final } \\
\text { Gap Conductance } \\
\left(\mathrm{kW} / \mathrm{m}^{2}-\mathrm{O}\right)\end{array}$ & $\begin{array}{l}\text { Initial/Final } \\
\text { Crack Factor }\end{array}$ \\
\hline 115 & $41.6 / 33.5$ & $1575 / 1310$ & $0.0321 / 0.0319$ & $4.02 / 3.48$ & $1.0 / 0.936$ \\
\hline 126 & $39.0 / 32.5$ & $1510 / 1297$ & $0.0326 / 0.0325$ & $3.93 / 3.36$ & $1.0 / 0.930$ \\
\hline 141 & $35.5 / 29.2$ & $1392 / 1187$ & $0.0325 / 0.0324$ & $3.62 / 3.13$ & $1.0 / 0.928$ \\
\hline
\end{tabular}




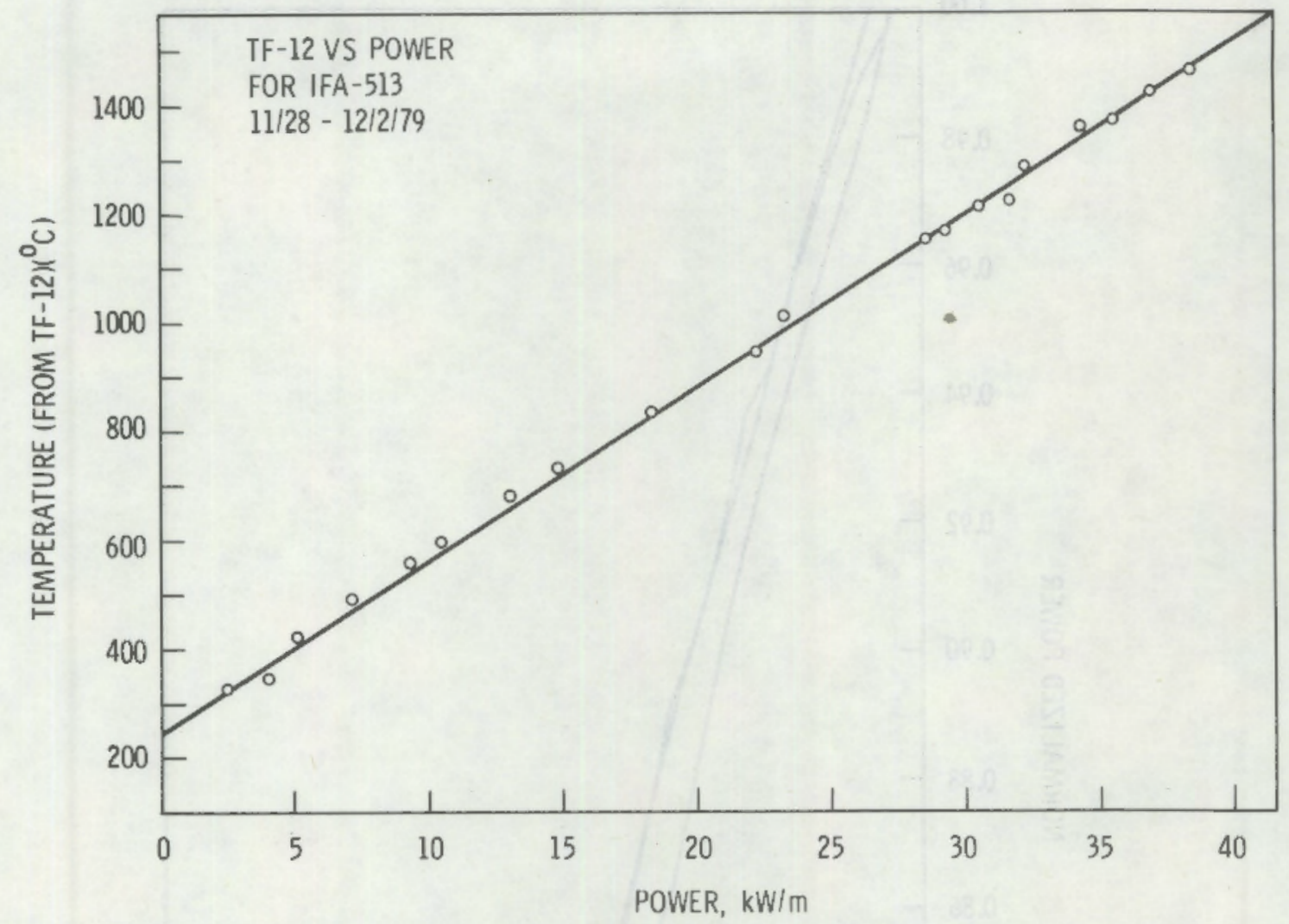

FIGURE 17. Steady-State Temperature Versus Power for IFA-513, Rod 6, (Upper Thermocouple-TF-12) on First Startup

is thus slightly lower. Also note that the variation in the inferred gap conductance between initial and final states is not insignificant. This same relative variation occurs in the crack factors ${ }^{(a)}$.

When normalized to the initial value, the power-versus-time plots for all 3 power drops are quite similar, as shown in Figure 18 . Run 126 is perhaps the "cleanest" in the sense of having a clean break to a final steady state

(a) The initial conductances for Models 1 and 2 were taken to be equal, which is why all initial crack factors are 1.0. 


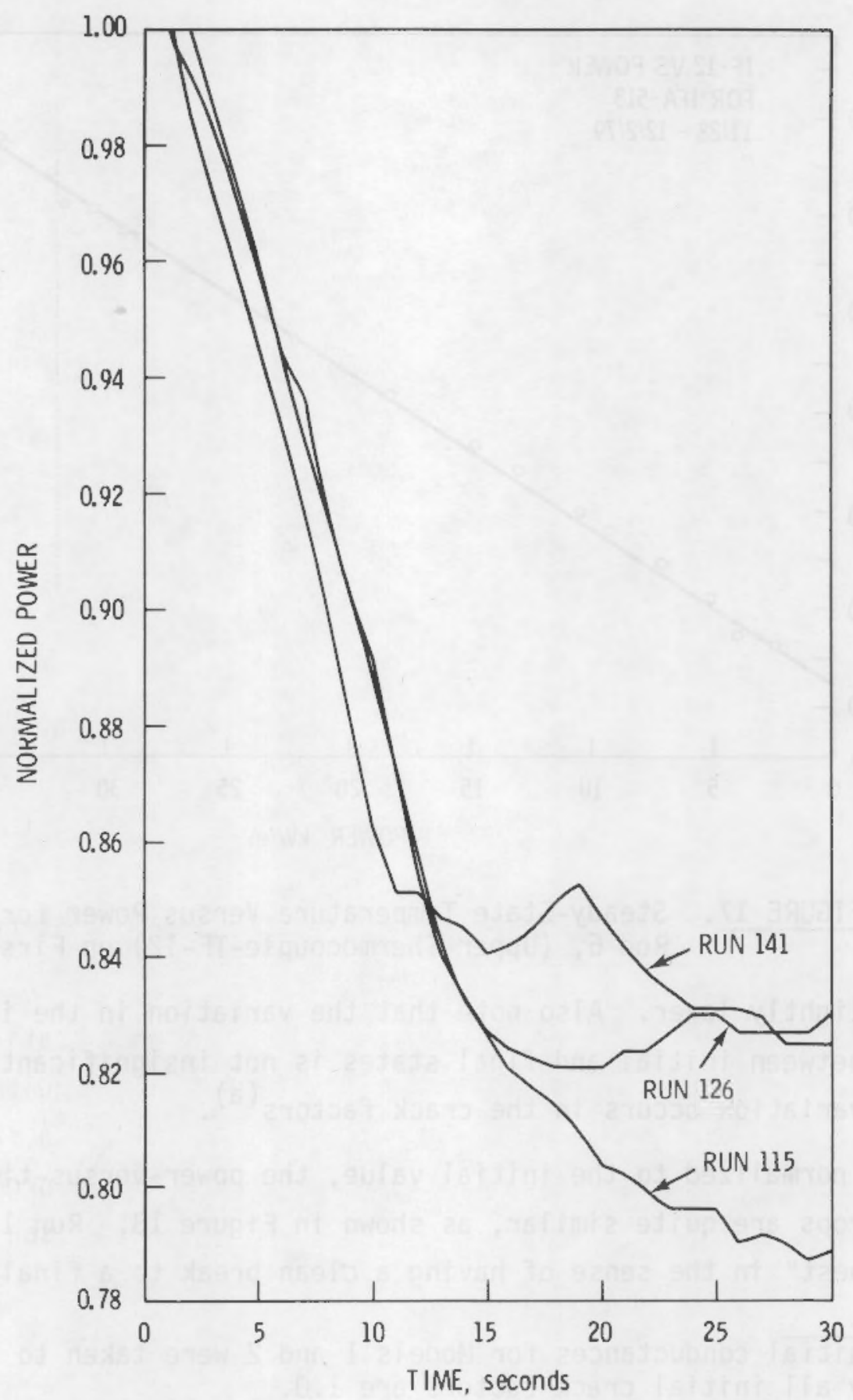

FIGURE 18. Relative Power History for the Transient Runs 


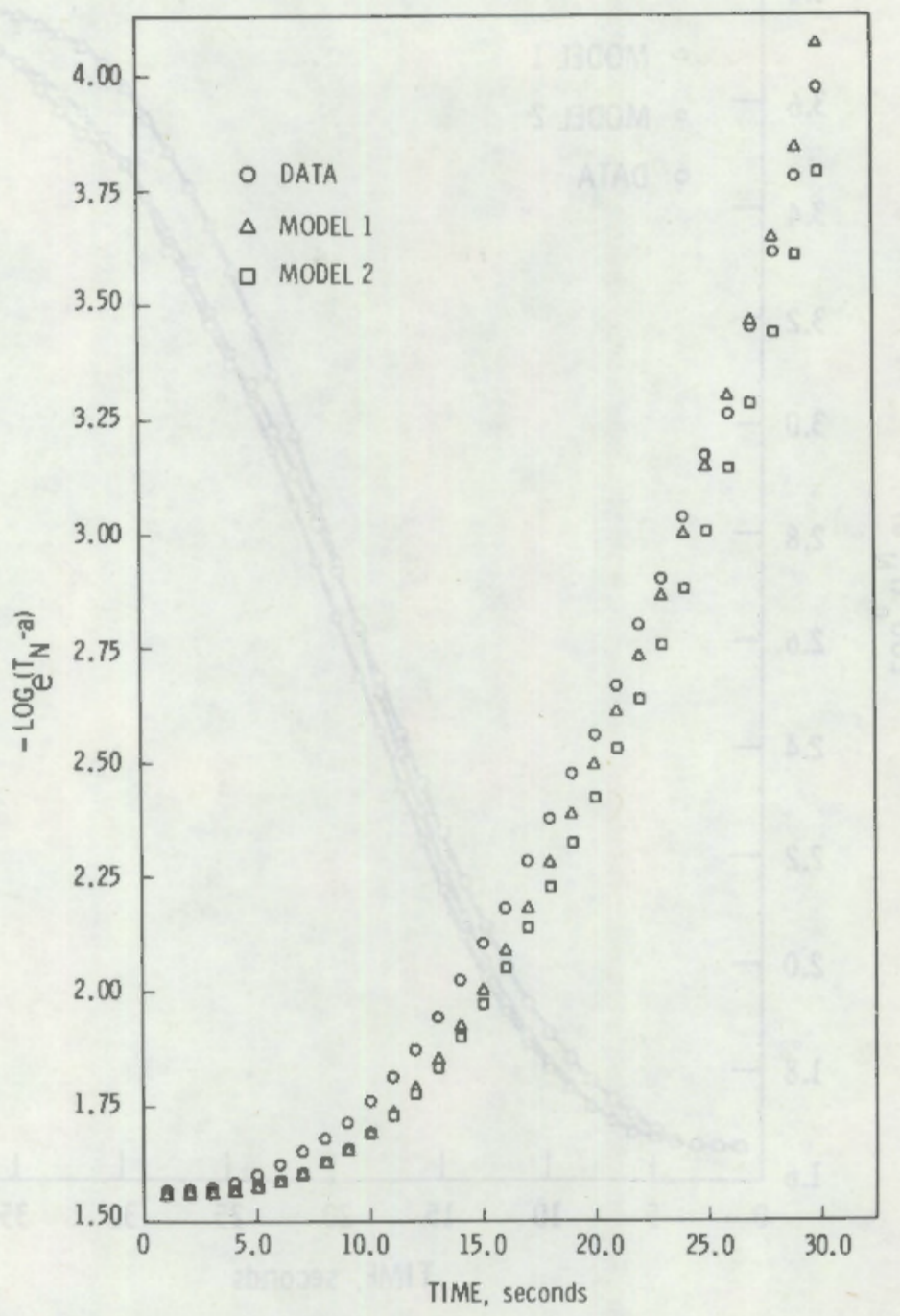

FIGURE 19. Data and Calculated Temperature Responses for the Upper Thermocouples of Rod 6 (Run 115)

the power drop. Run 115 does not break over very sharply at the lower end, and Run 141 has a bad hump at the lower end before the final steady state is attained. Of the three, Run 126 will probably yield the most definitive data.

The corresponding rod 6 temperature response and comparative Model $1 /$ Model 2 calculations are shown in Figures 19 to 21 . It is quite clear that in all 3 cases Model 2 follows the trend (curvature) of the data far better than Model 1. The offset that Model 2 develops from the data is probably due to an incorrect choice of thermocouple time constant. The comparison between models 


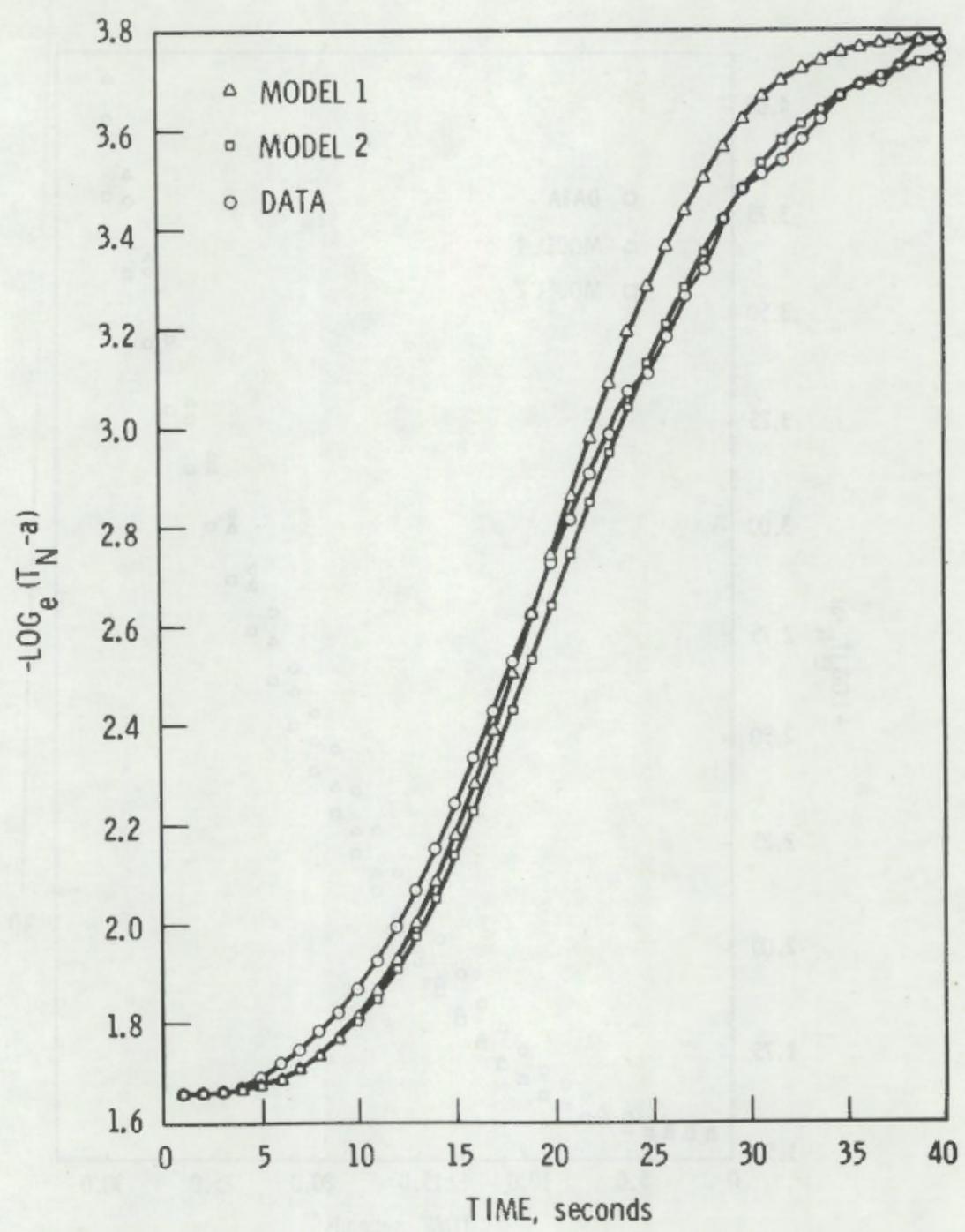

FIGURE 20. Data and Calculated Temperature Response for Rod 6, Upper Thermocouple, Run 126

and data is made more dramatic in Figure 22, where we show the data-minuscalculated differences for Run 141 (Figure 21). In the range where a constant difference should be expected (from 12 to $17 \mathrm{sec}$ ), Model 2 evidences a constant difference, whereas Model 1 does not ${ }^{(a)}$.

(a) The increase in the data-minus-calculated difference after about 17 seconds is due to the hump in the power history, combined with an incorrect choice in the thermocouple time constant. If the plots were carried far enough, the differences (for both models) would fall to nearly zero, as the final steady state was approached. 


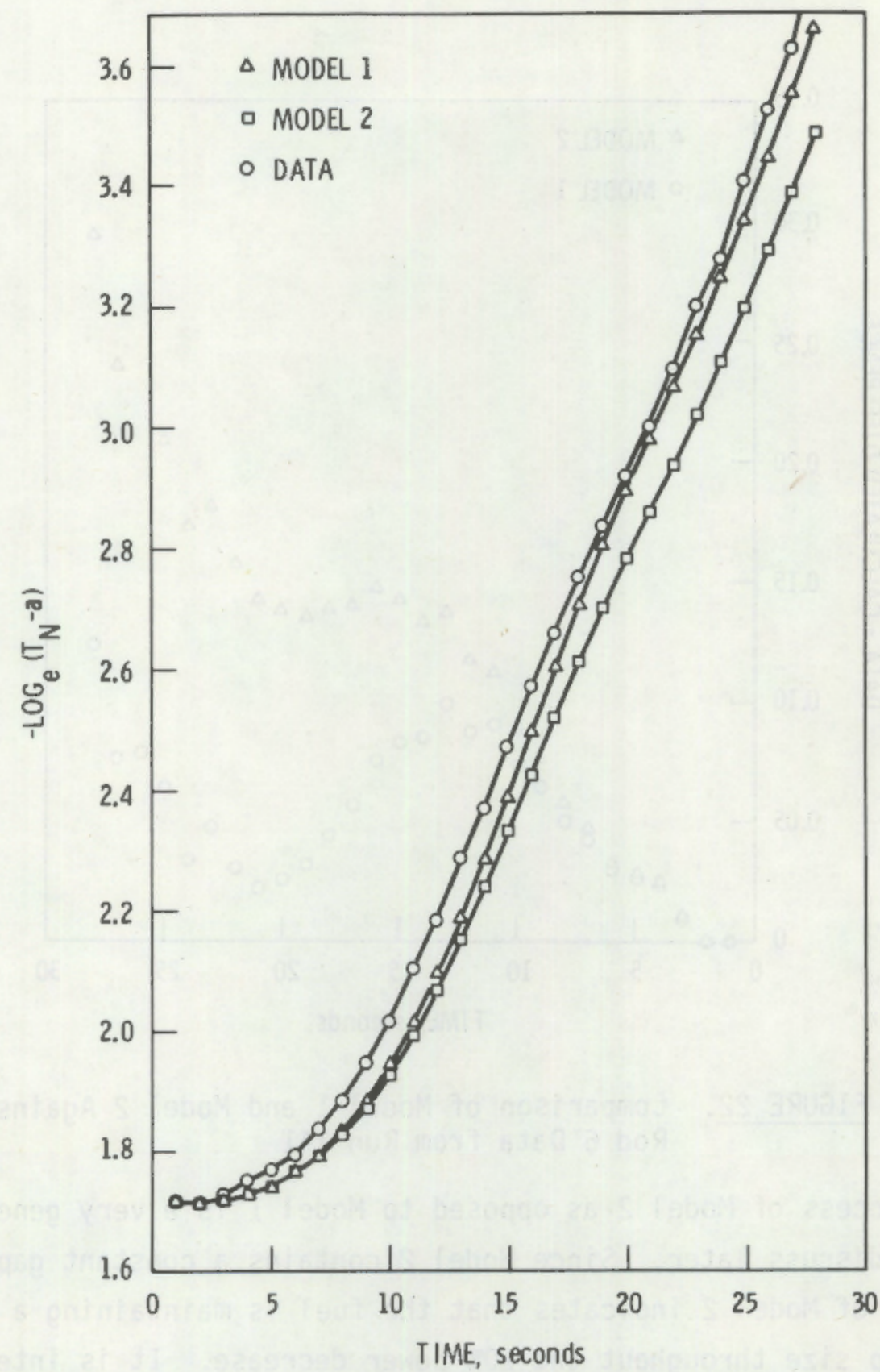

FIGURE 21. Data and Calculated Response for Rod 6, Upper Thermocouple, Run 141 


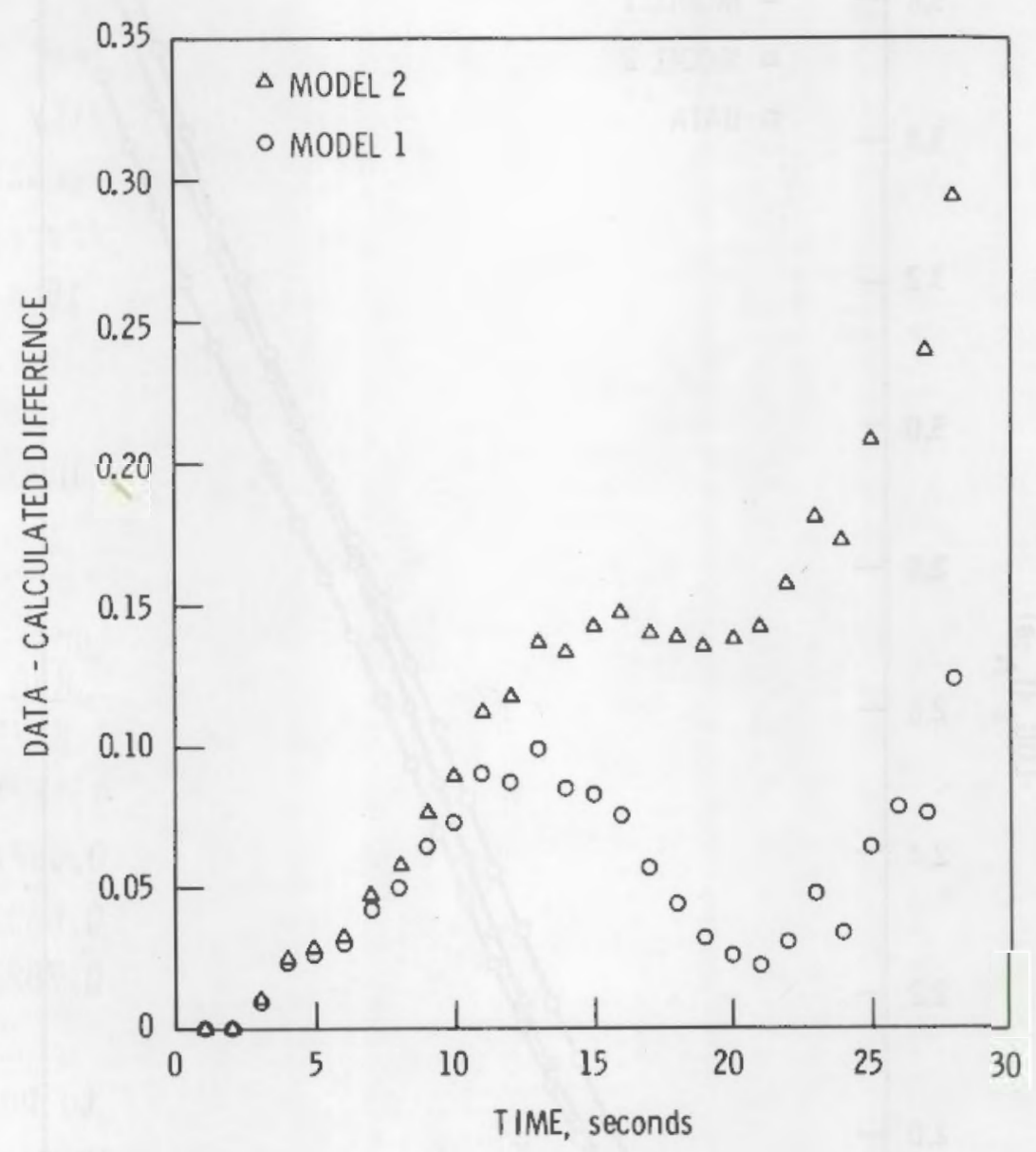

FIGURE 22. Comparison of Model 1 and Model 2 Against Rod 6 Data from Run 141

This success of Model 2 as opposed to Model 1 is a very general result, as we shall discuss later. Since Model 2 contains a constant gap conductance, this success of Mode 12 indicates that the fuel is maintaining a very constant effective gap size throughout the $20 \%$ power decrease. It is interesting to speculate what this gap size might be. This can be done by running a survey of Model 2 cases, each having a different conductance, but each having a crack factor function matching the initial/final states of the rod. 


\section{SURVEY CALCULATIONS}

To span the range of probable gap conductances for upper thermocouple location we chose a range of 2.0 to $8.0 \mathrm{~kW} / \mathrm{m}^{2}-{ }^{\circ} \mathrm{C}$. The corresponding initial/final crack factors generated by MWRAM are shown in Table 9, for all 3 transient runs. The crack factor values at corresponding conductances for all 3 runs are quite similar. Note that the crack factor for a gap conductance of $2.0 \mathrm{~kW} / \mathrm{m}^{2}-{ }^{\circ} \mathrm{C}$ is much greater than 1.0. Since there is no clear mechanism for such a large enhancement of the effective fuel conductivity it is probable that the conductance is truly in the range of 3.0 to 4.0 or greater. This is borne out by the difference plots (data-minus calculated differences of $\ln \left(T_{N}-a\right)$ plot-versus-time), which are shown for each of the 15 survey calculations in Figures 23 to 37.

TABLE 9. Crack Factors for Model 2 for TF 12 Using Specific Values of Gap Conductance

Gap Conductance value,

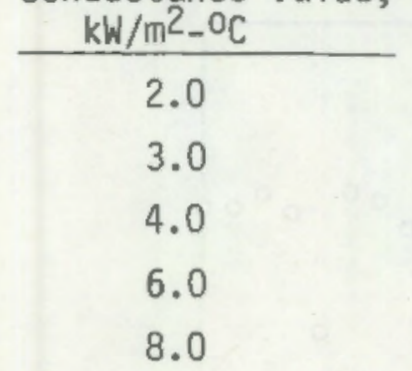

\begin{tabular}{l}
\hline Run 115 \\
\hline $1.59 / 1.50$ \\
$1.16 / 1.08$ \\
$1.00 / 0.938$ \\
$0.874 / 0.819$ \\
$0.818 / 0.767$
\end{tabular}
Initial/Final Crack Factors

Figures 23-27, for Run 115, show the gap conductance to be in the range of 2.5 to 3.5. Figures 28-32, for Run 126, indicate approximately the same range. Figures 33-37, for Run 141, seem to indicate a higher range, from about 4.0 to 8.0 . However, this may be partially due to the hump in the power history; thus it could be an artifact of the power history ${ }^{(a)}$.

(a) Another confusing factor is the choice of thermocouple time constant. Notice that in a11 the model 2 comparisons to data (Figures 23-37), a constant difference of 0.15 is established. For a 1 these comparisons, an assumed thermocouple time constant of $1 / 1.5 \mathrm{sec}^{-1}$ was used. However, consider Figure 38, where a time constant of $1 / 0.2$ is used; the comparison to data is much better over a very long time range. 


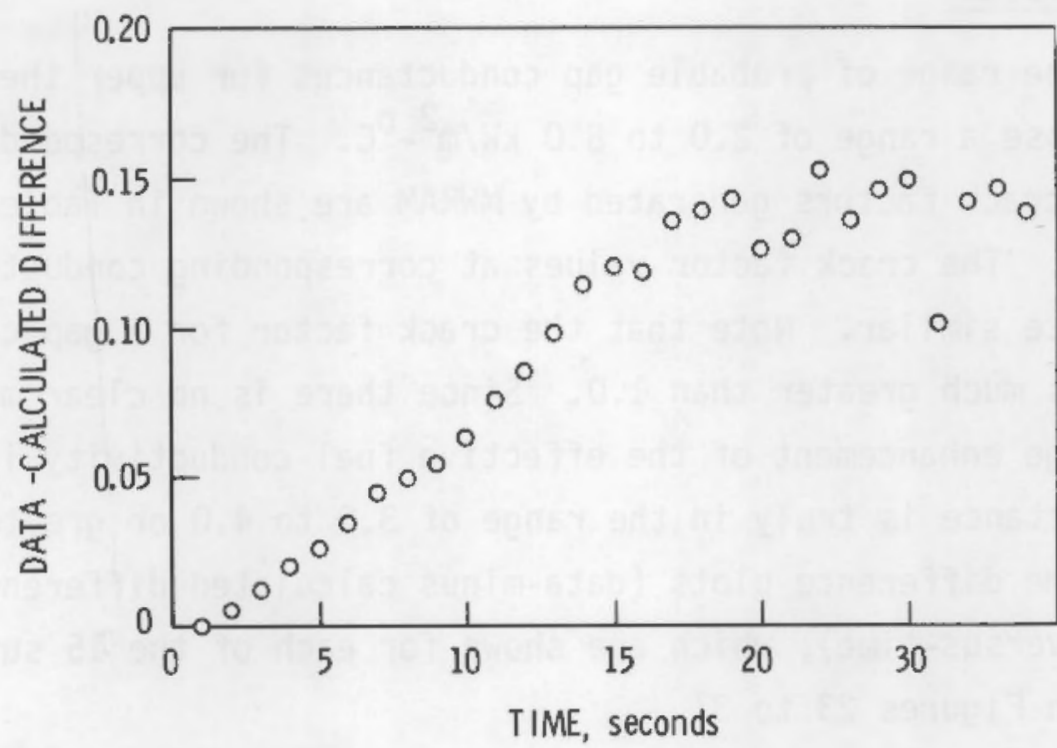

FIGURE 23. Model 2 Compared to Rod 6 Data for Run 115 (Assuming Gap Conductance $=2.0 \mathrm{~kW} / \mathrm{m}^{2}-{ }^{\circ} \mathrm{C}$ )

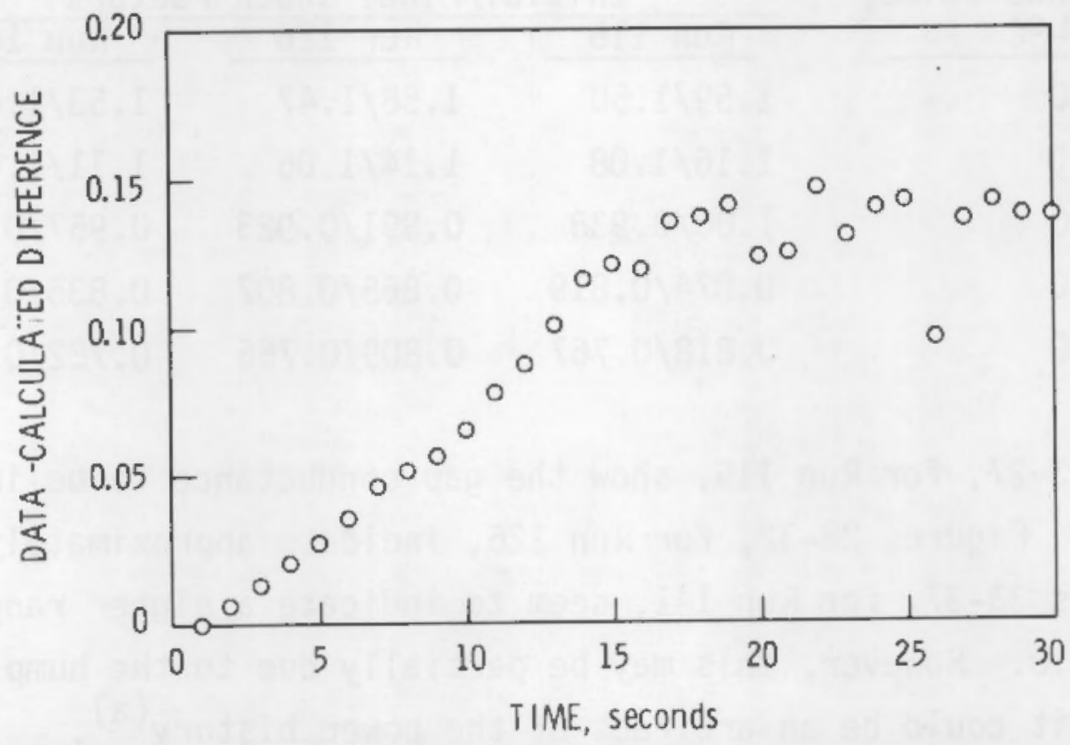

FIGURE 24. Mode1 2 Compared to Rod 6 Data from Run 115 (Assuming Gap Conductance $=3.0 \mathrm{~kW} / \mathrm{m}^{2}-{ }^{\circ} \mathrm{C}$ ) 


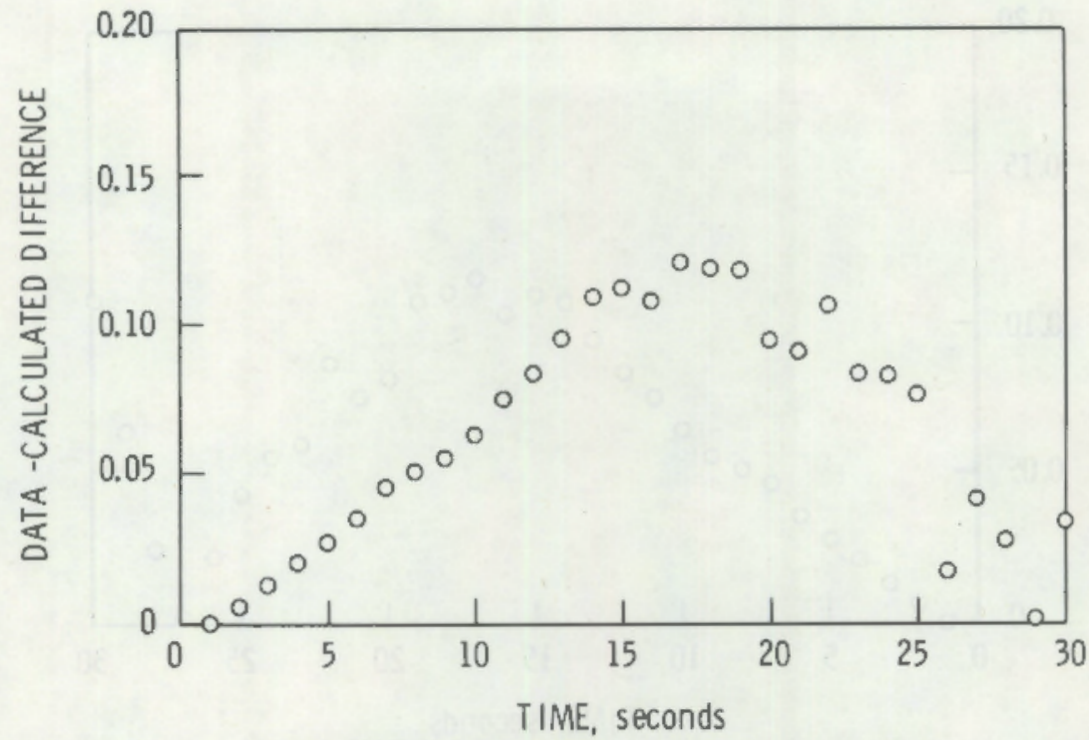

FIGURE 25. Model 2 Compared to Rod 6 Data from Run 115 (Assuming Gap Conductance $=4.0 \mathrm{~kW} / \mathrm{m}^{2}{ }^{\circ} \mathrm{C}$ )

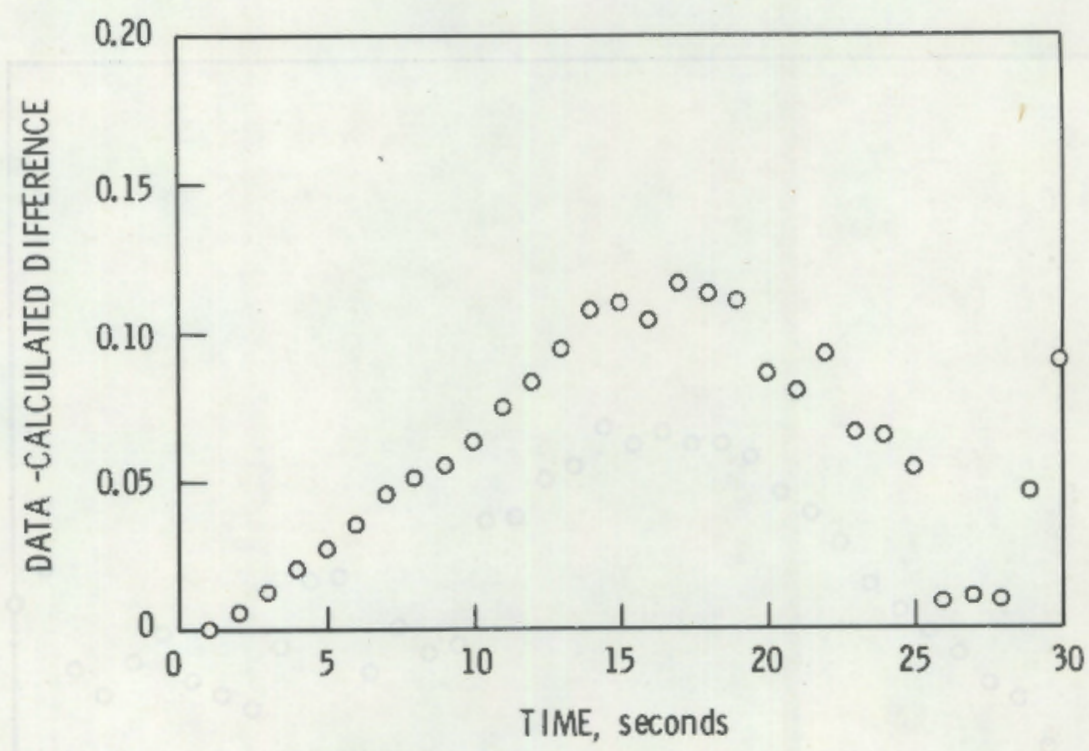

FIGURE 26. Model 2 Compared to Rod 6 Data From Run 115 (Assuming Gap Conductance $=6.0 \mathrm{~kW} / \mathrm{m}^{2}-{ }^{0} \mathrm{C}$ ) 


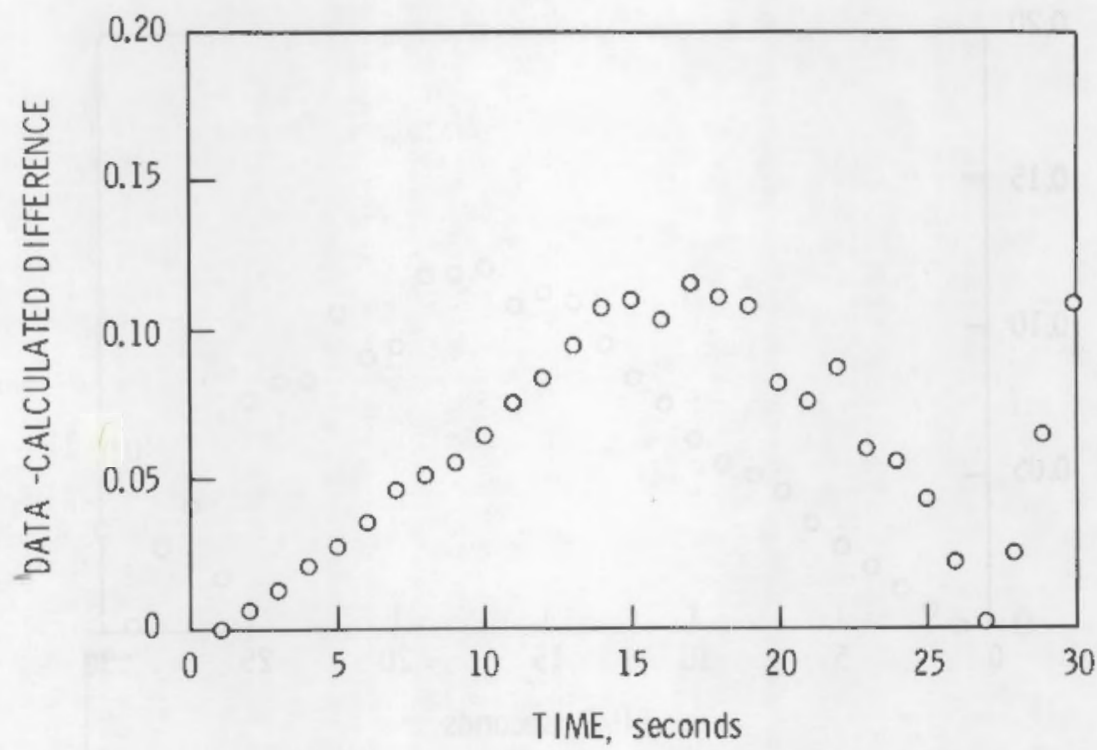

FIGURE 27. Model 2 Compared to Rod 6 Data from Run 115 (Assuming Gap Conductance $=8.0 \mathrm{~kW} / \mathrm{m}^{2}-{ }^{\circ} \mathrm{C}$ )

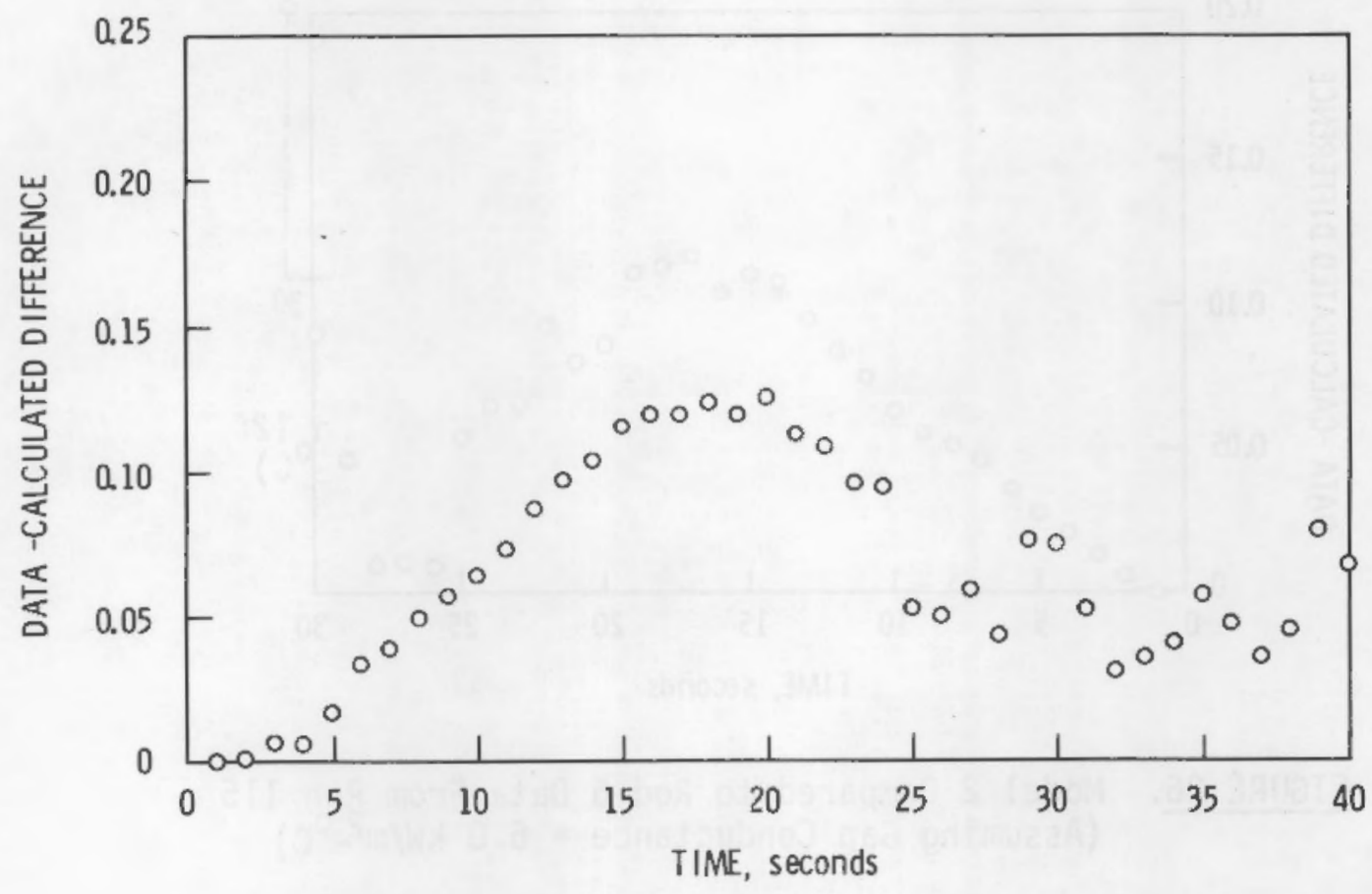

FIGURE 28. Model 2 Compared to Rod 6 Data from Run 126 (Assuming Gap Conductance $=2.0 \mathrm{~kW} / \mathrm{m}^{2}{ }^{\circ} \mathrm{C}$ ) 


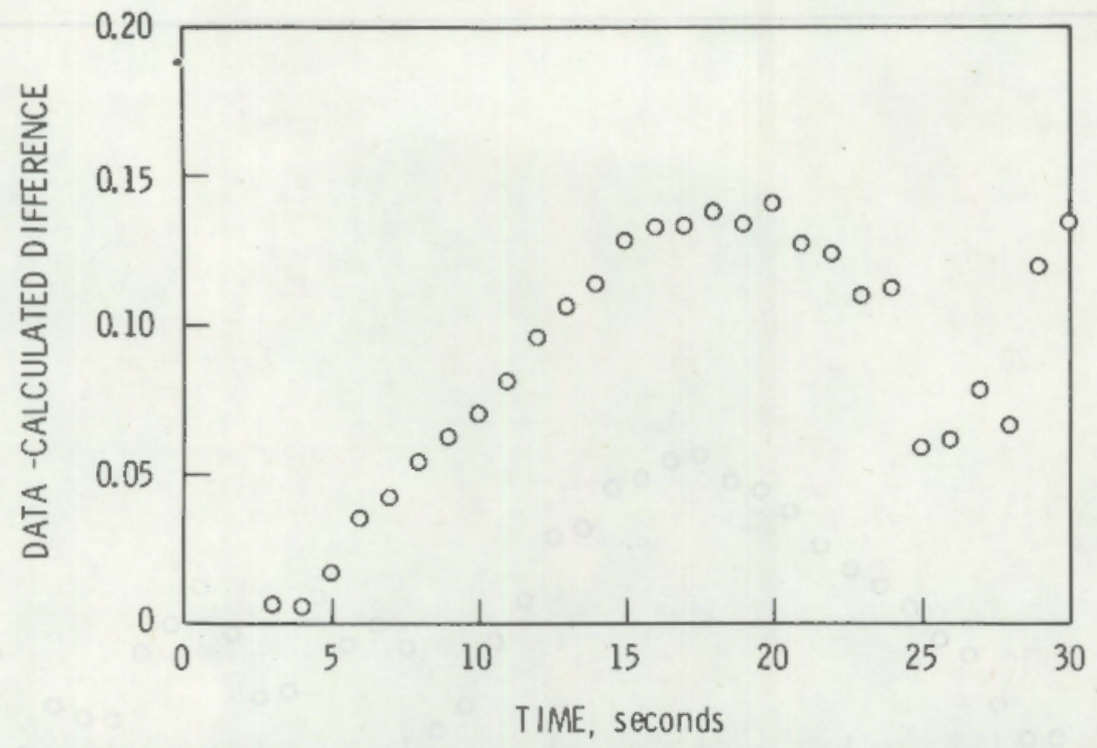

FIGURE 29. Mode1 2 Compared to Rod 6 Data from Run 126 (Assuming Gap Conductance $=3.0 \mathrm{~kW} / \mathrm{m}^{2}{ }^{\circ} \mathrm{C}$ )

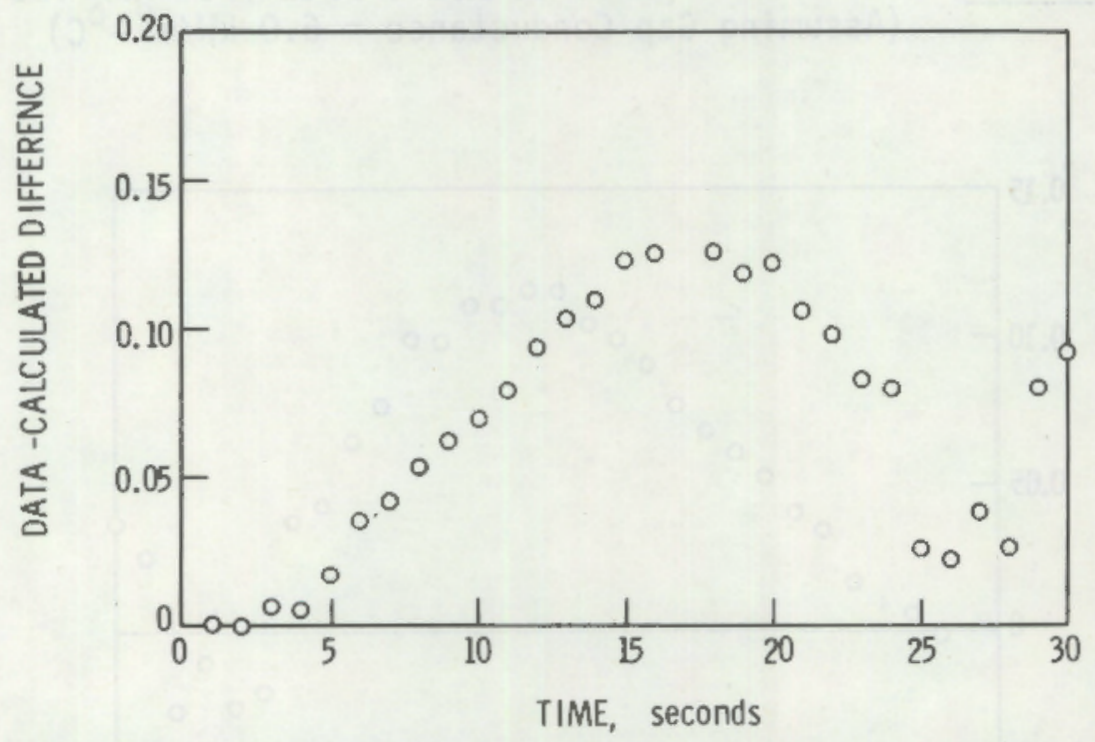

FIGURE 30. Model 2 Compared to Rod 6 Data for Ryn 126 (Assuming Gap Conductance $=4.0 \mathrm{~kW} / \mathrm{m}^{2}{ }^{\circ} \mathrm{C}$ ) 


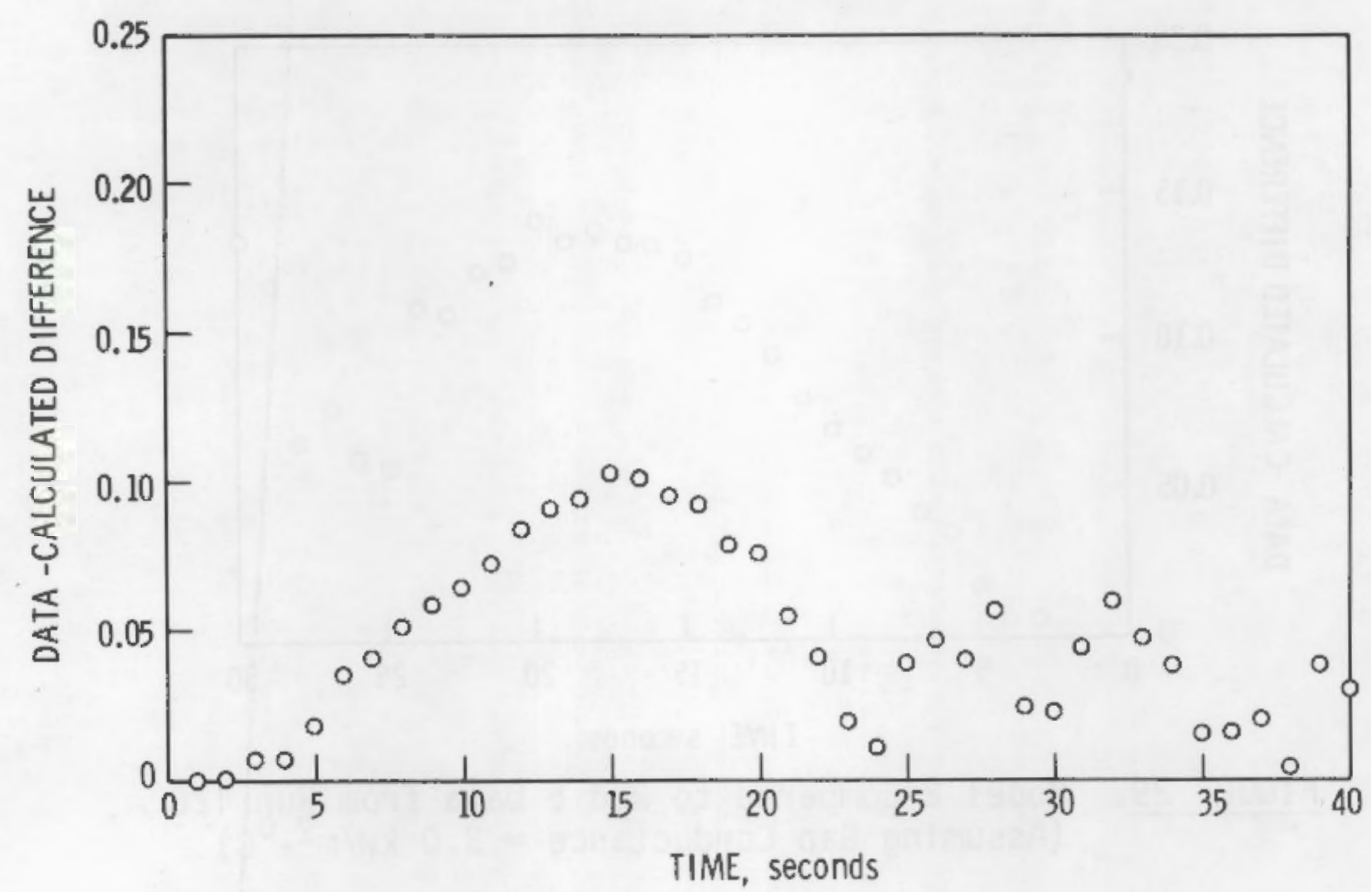

FIGURE 31. Model 2 Compared to Rod 6 Data from Run 126 (Assuming Gap Conductance $=6.0 \mathrm{~kW} / \mathrm{m}^{2}-{ }^{\circ} \mathrm{C}$ )

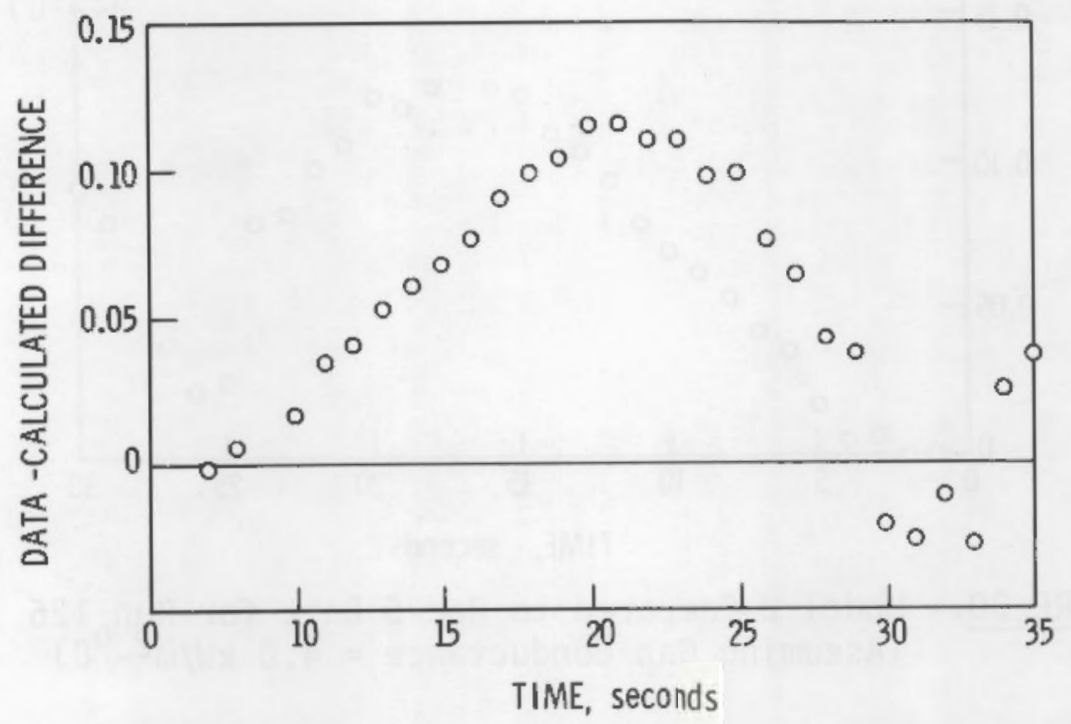

FIGURE 32. Model 2 Compared to Rod 6 Data from Run 126 (Assuming Gap Conductance $=8.0 \mathrm{~kW} / \mathrm{m}^{2}-{ }^{\circ} \mathrm{C}$ ) 


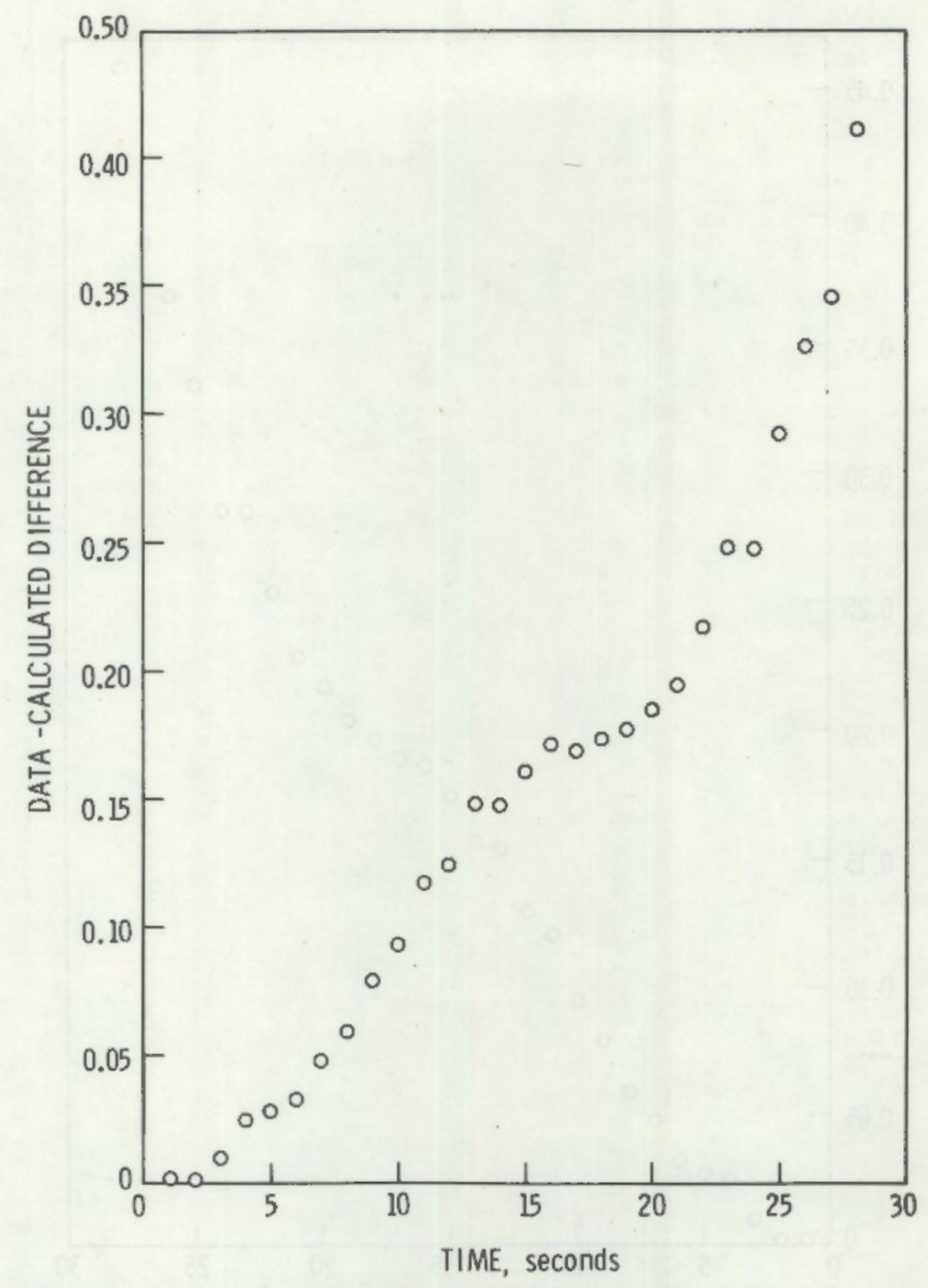

FIGURE 33. Model 2 Compared to Rod 6 Data from Run 141 (Assuming Gap Conductance $=2.0 \mathrm{~kW} / \mathrm{m}^{2}-\mathrm{C}$ ) 


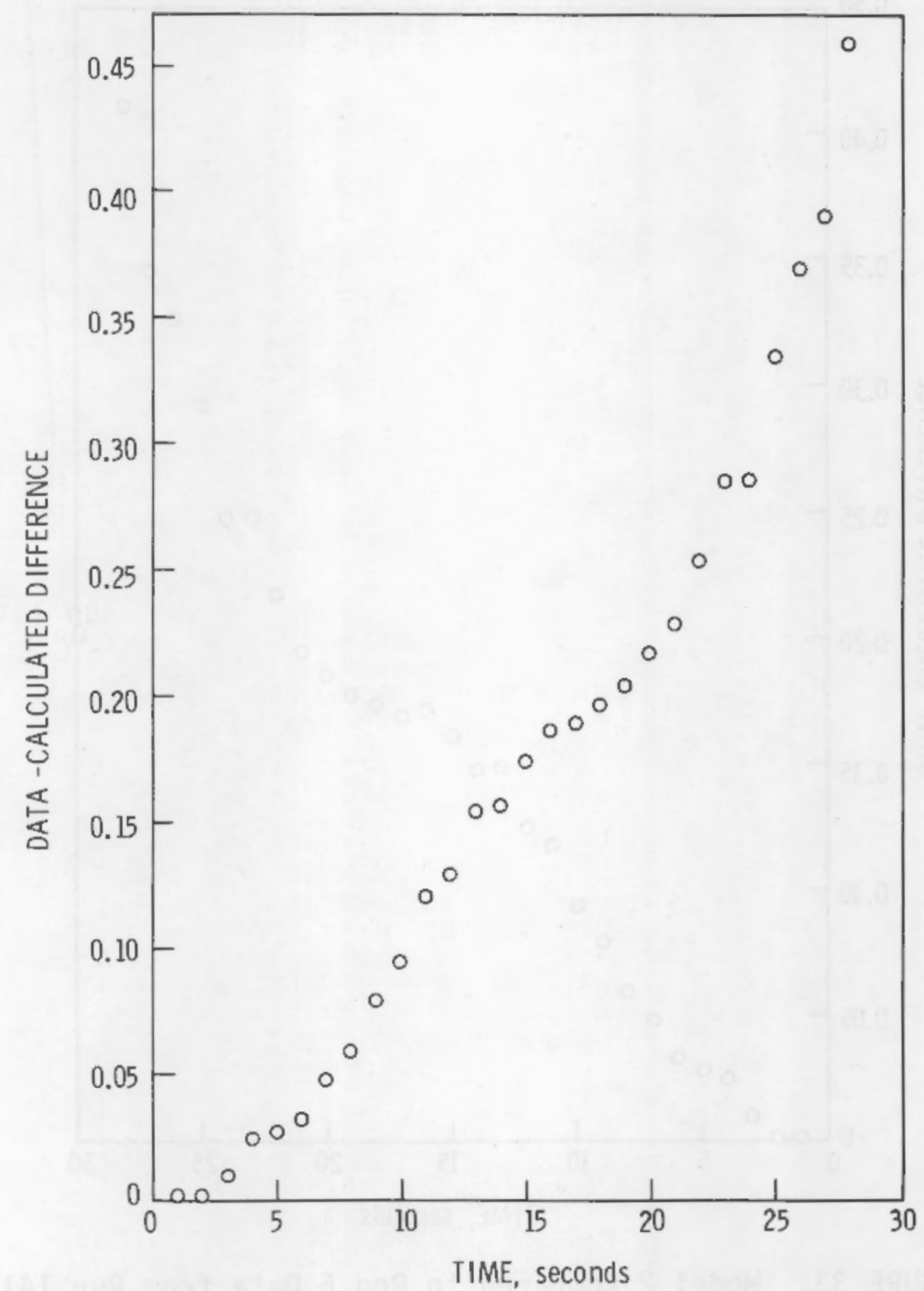

FIGURE 34. Mode1 2 Compared to Rod 6 Data from Run 141 (Assuming Gap Conductance $=3.0 \mathrm{~kW} / \mathrm{m}^{2}-{ }^{\circ} \mathrm{C}$ ) 


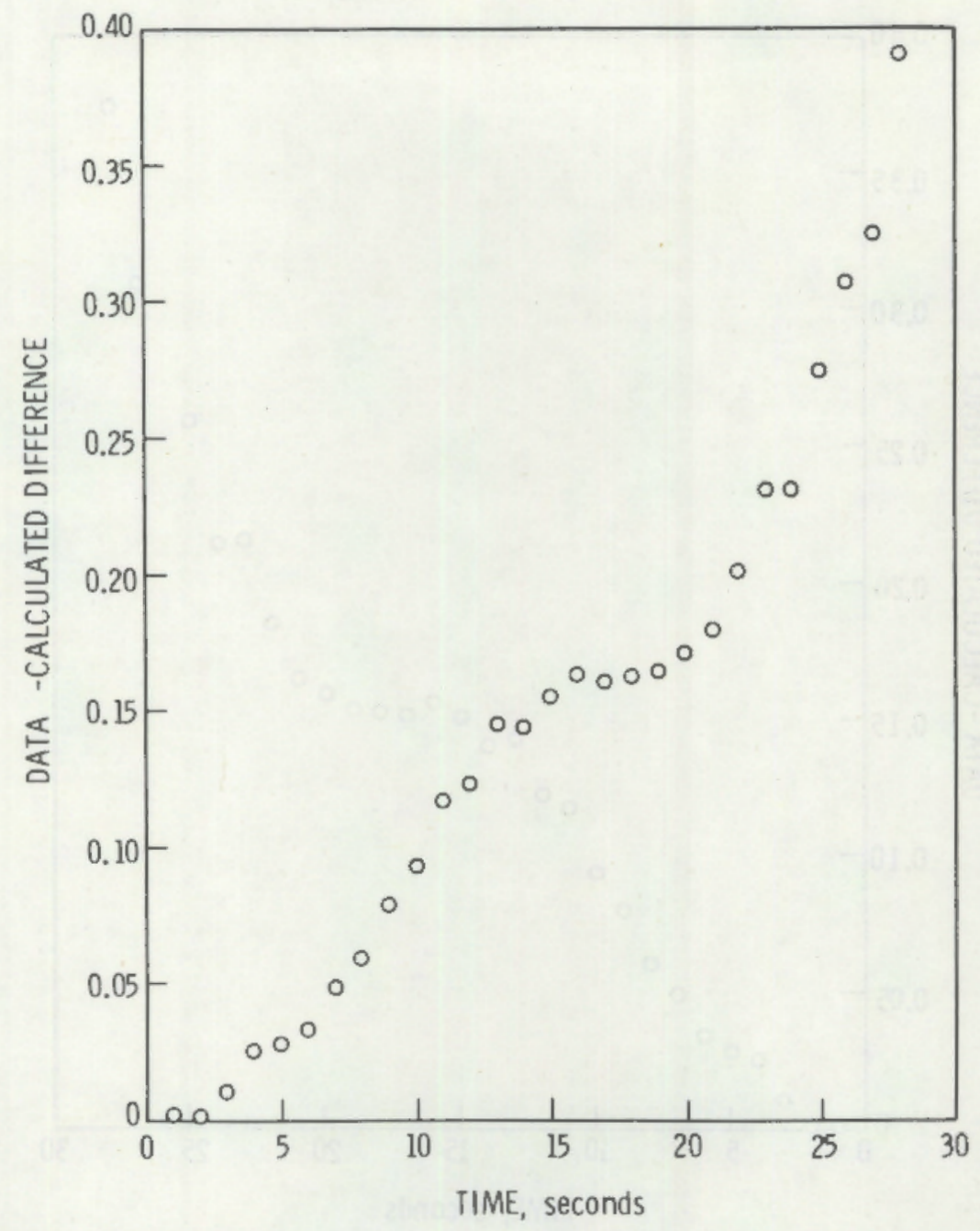

FIGURE 35. Model 2 Compared to Rod 6 Data from Run 141 (Assuming Gap Conductance $=4.0 \mathrm{~kW} / \mathrm{m}^{2}-\mathrm{C}$ ) 


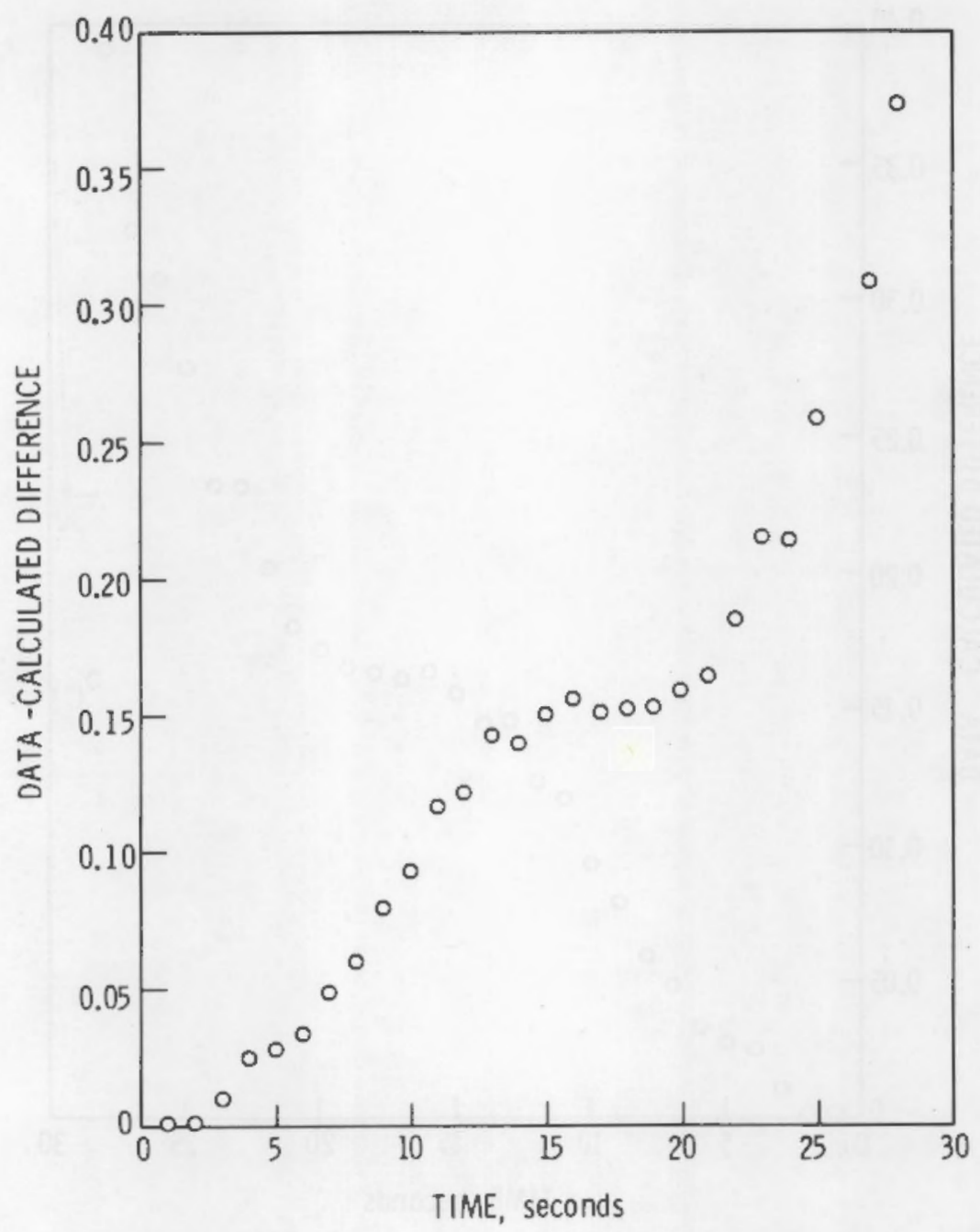

FIGURE 36. Model 2 Compared to Rod 6 Data from Run 141 (Assuming Gap Conductance $=6.0 \mathrm{~kW} / \mathrm{m}^{2-} \mathrm{C}$ ) 


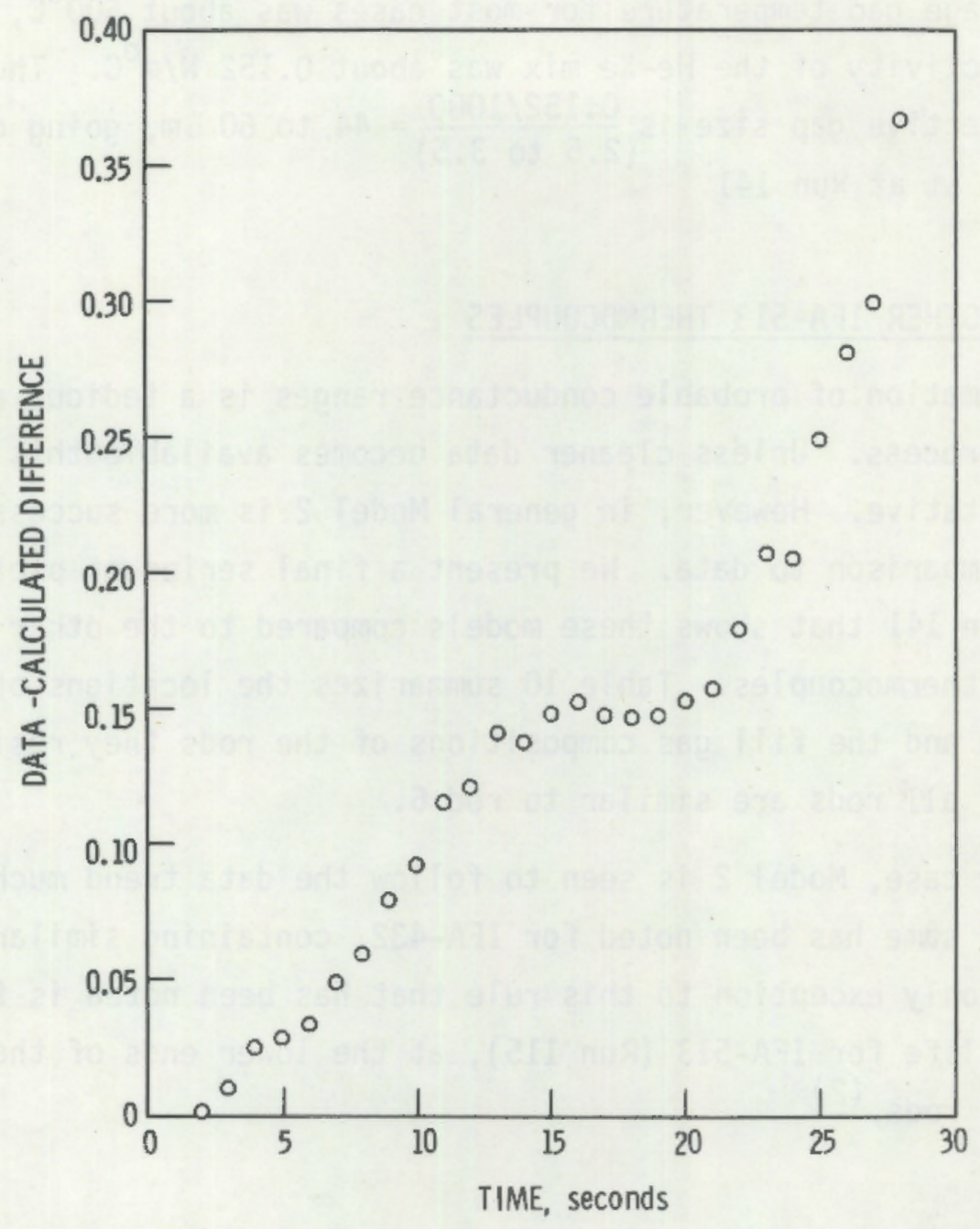

FIGURE 37. Model 2 Compared to Data from Run 141 (Assuming Gap Conductance $=8.0 \mathrm{~kW} / \mathrm{m}^{2}-{ }^{\circ} \mathrm{C}$ ) 
The average gap temperature for most cases was about $500^{\circ} \mathrm{C}$, so that thermal conductivity of the He-Xe mix was about $0.152 \mathrm{~W} / \mathrm{m}^{\circ} \mathrm{C}$. Thus, the estimated effective gap size is $\frac{0.152 / 1000}{(2.5 \text { to } 3.5)}=44$ to $60 \mu \mathrm{m}$, going down to perhaps 20-40 $\mu \mathrm{m}$ at Run 141 .

\section{EXTENSION TO OTHER IFA-513 THERMOCOUPLES}

The estimation of probable conductance ranges is a tedious and highly speculative process. Unless cleaner data becomes available this process will not be quantitative. However, in general Model 2 is more successful than Model 1 in comparison to data. We present a final series of plots (Figures 39-47) for run 141 that shows these models compared to the other 9 surviving IFA-513 fuel thermocouples. Table 10 summarizes the locations of these thermocouples and the fill gas compositions of the rods they reside in. The dimensions of all rods are similar to rod 6 .

In every case, Model 2 is seen to follow the data trend much better than Mode1 1. The same has been noted for IFA-432, containing similar rods at high burnup. The only exception to this rule that has been noted is for the very beginning of life for IFA-513 (Run 115), at the lower ends of the helium-filled rods. ${ }^{(2)}$ 


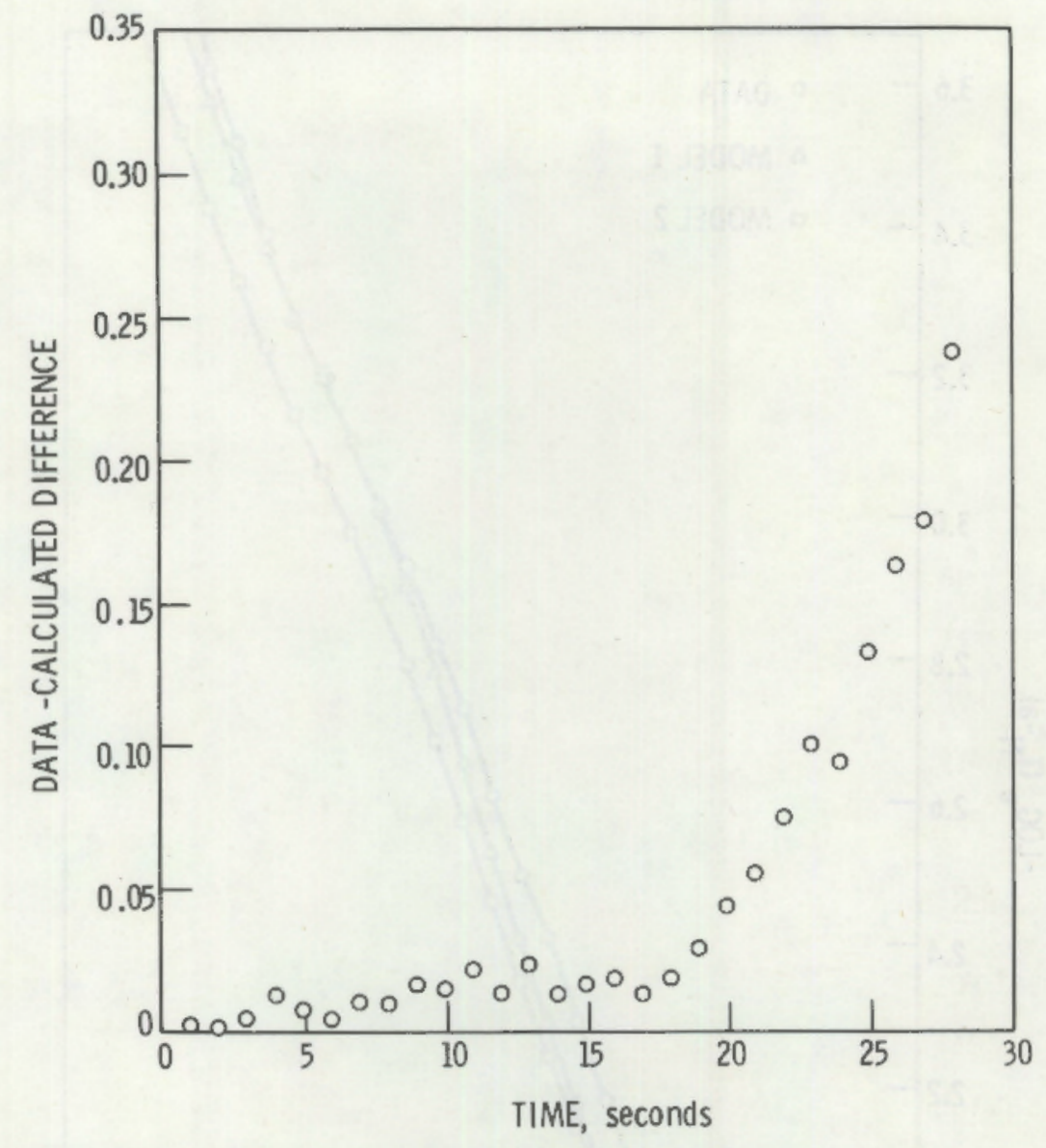

FIGURE 38. Model 2 Compared to Data from Run 141 (Gap Conductance $=8.0 \mathrm{~kW} / \mathrm{m}^{2}-{ }^{\circ} \mathrm{C}$ ) Assuming Thermocouple Time Constant $=1 / 0.2 \mathrm{sec}^{-1}$ 


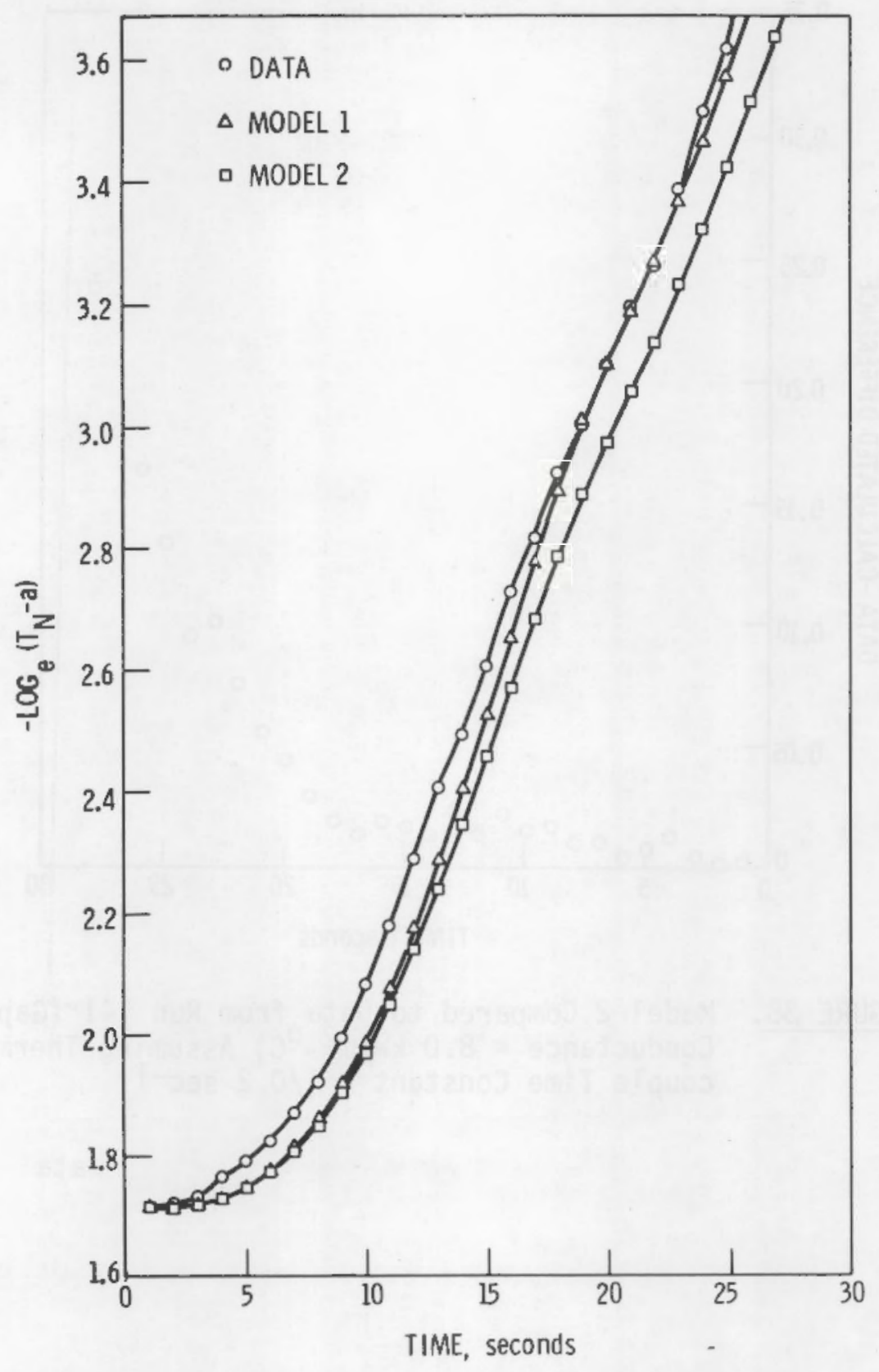

FIGURE 39. Models 1 and 2 Compared to Rod 1 (Lower Thermocouple Data for Run 141) 


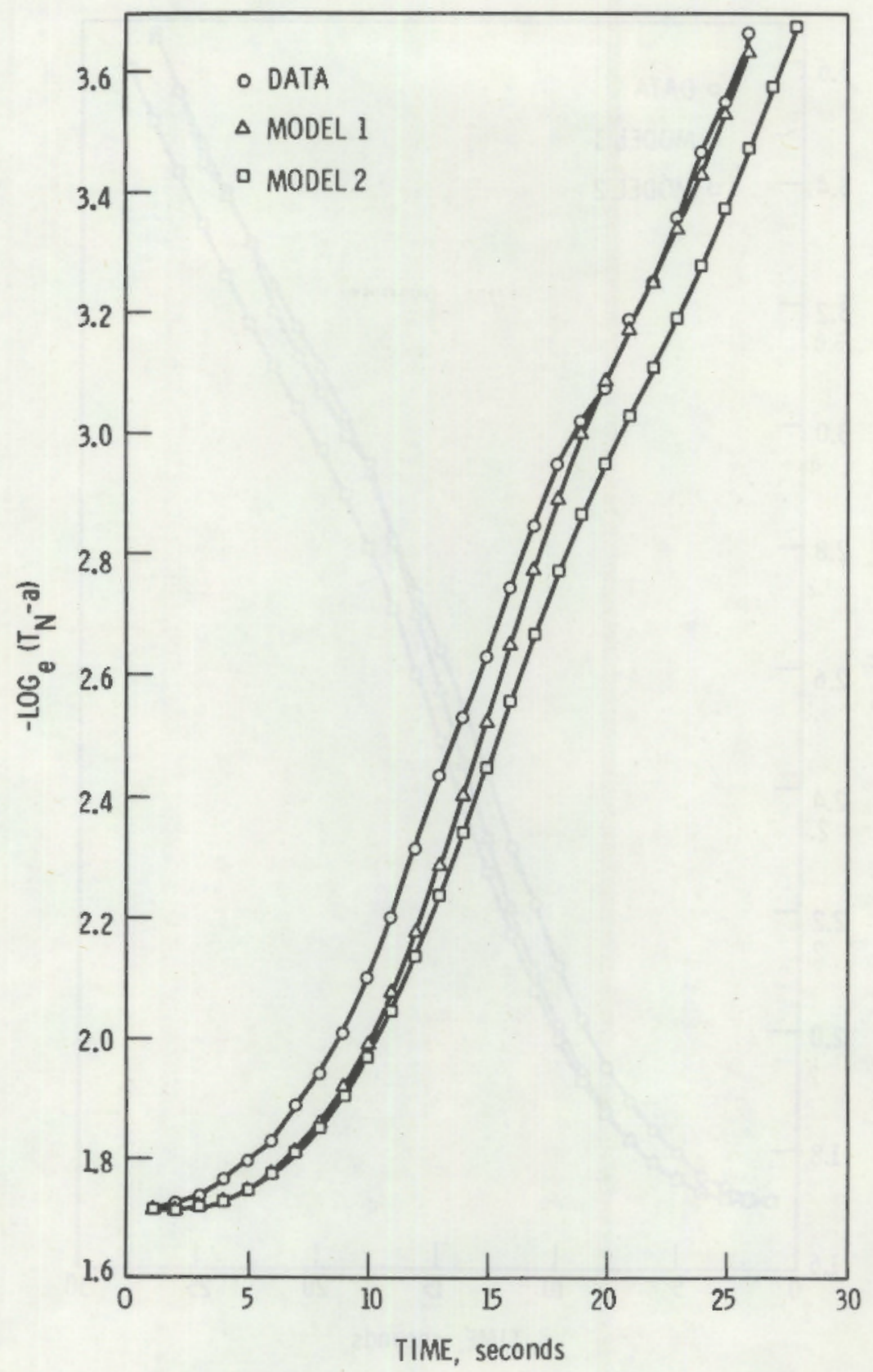

FIGURE 40. Models 1 and 2 Compared to Rod 1 Data (Upper Thermocouple) from Run 141 


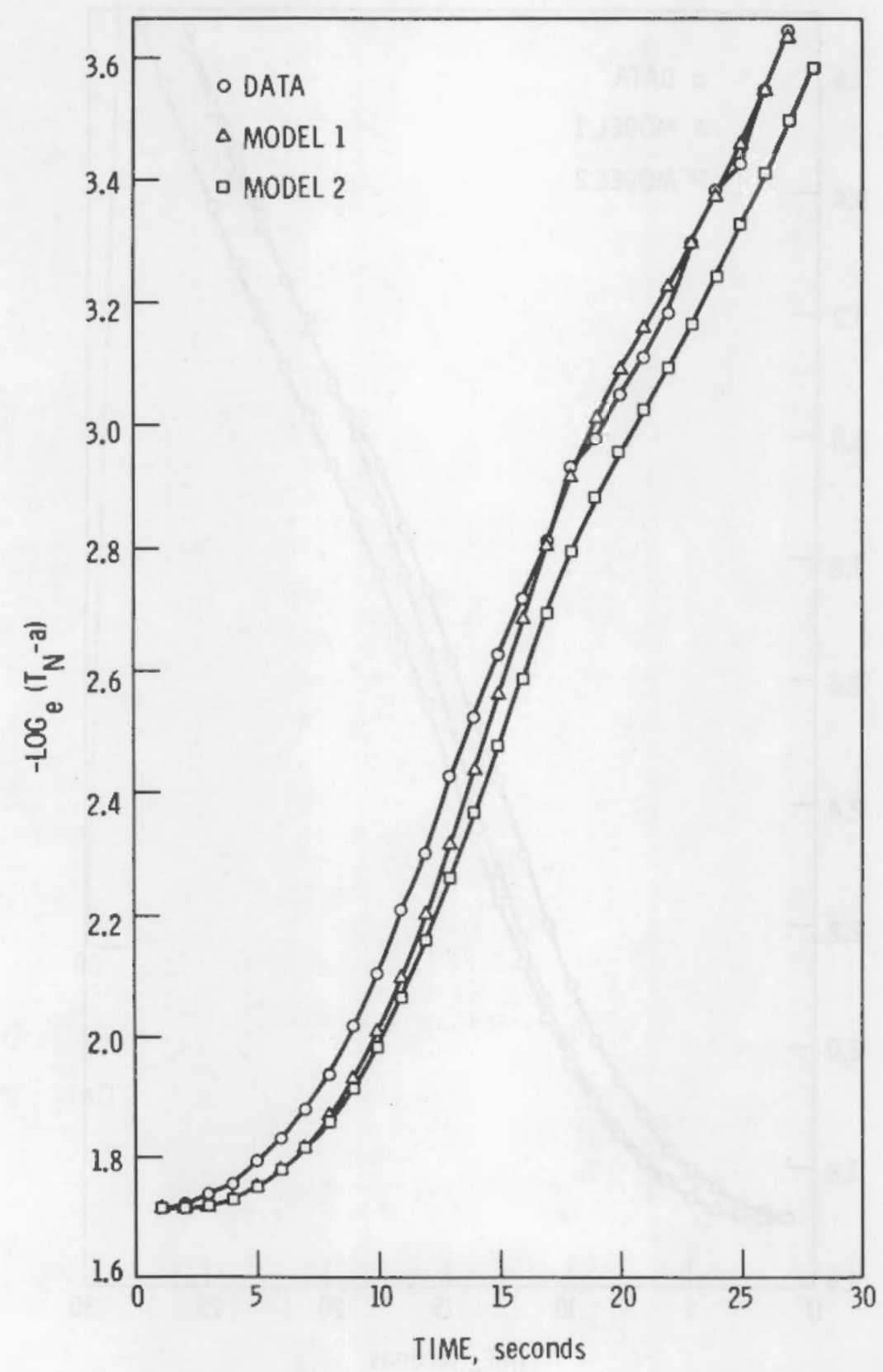

FIGURE 41. Models 1 and 2 Compared to Rod 2 Data (Lower Thermocouple) for Run 141 


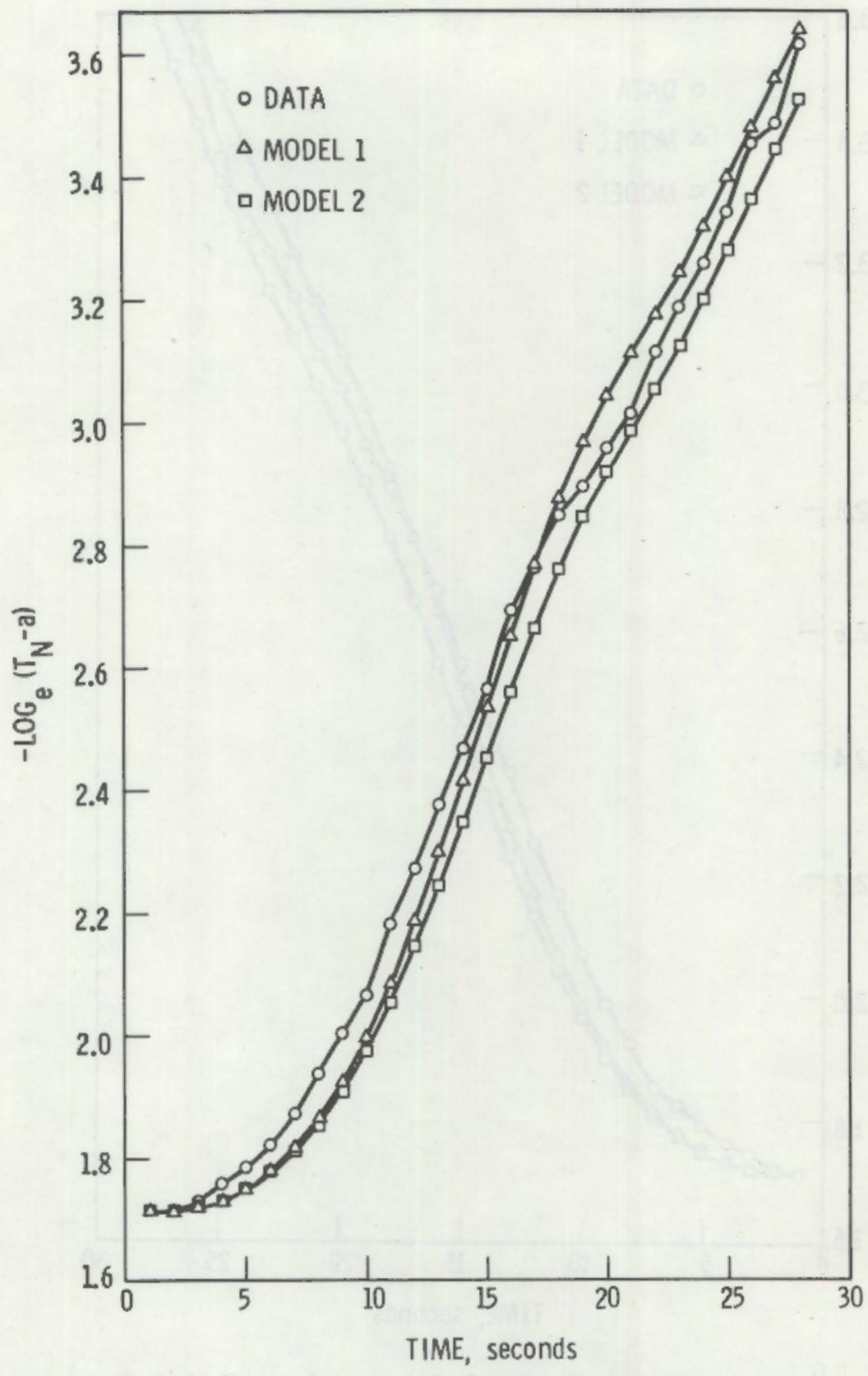

FIGURE 42. Models 1 and 2 Compared to Rod 3 Data (Lower Thermocouple) for Run 141 


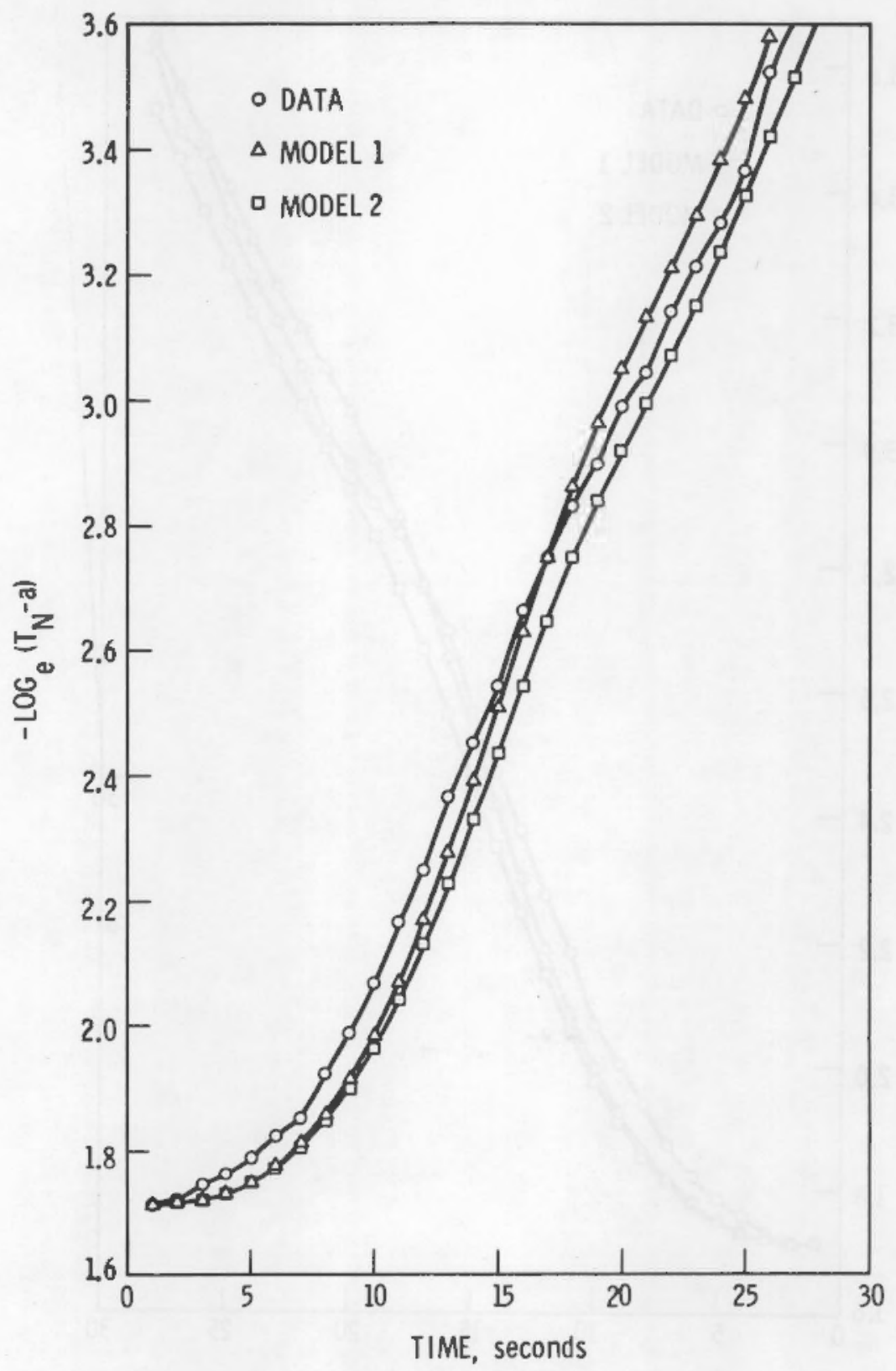

FIGURE 43. Models 1 and 2 Compared to Rod 4 Data (Lower Thermocouple) for Run 141 


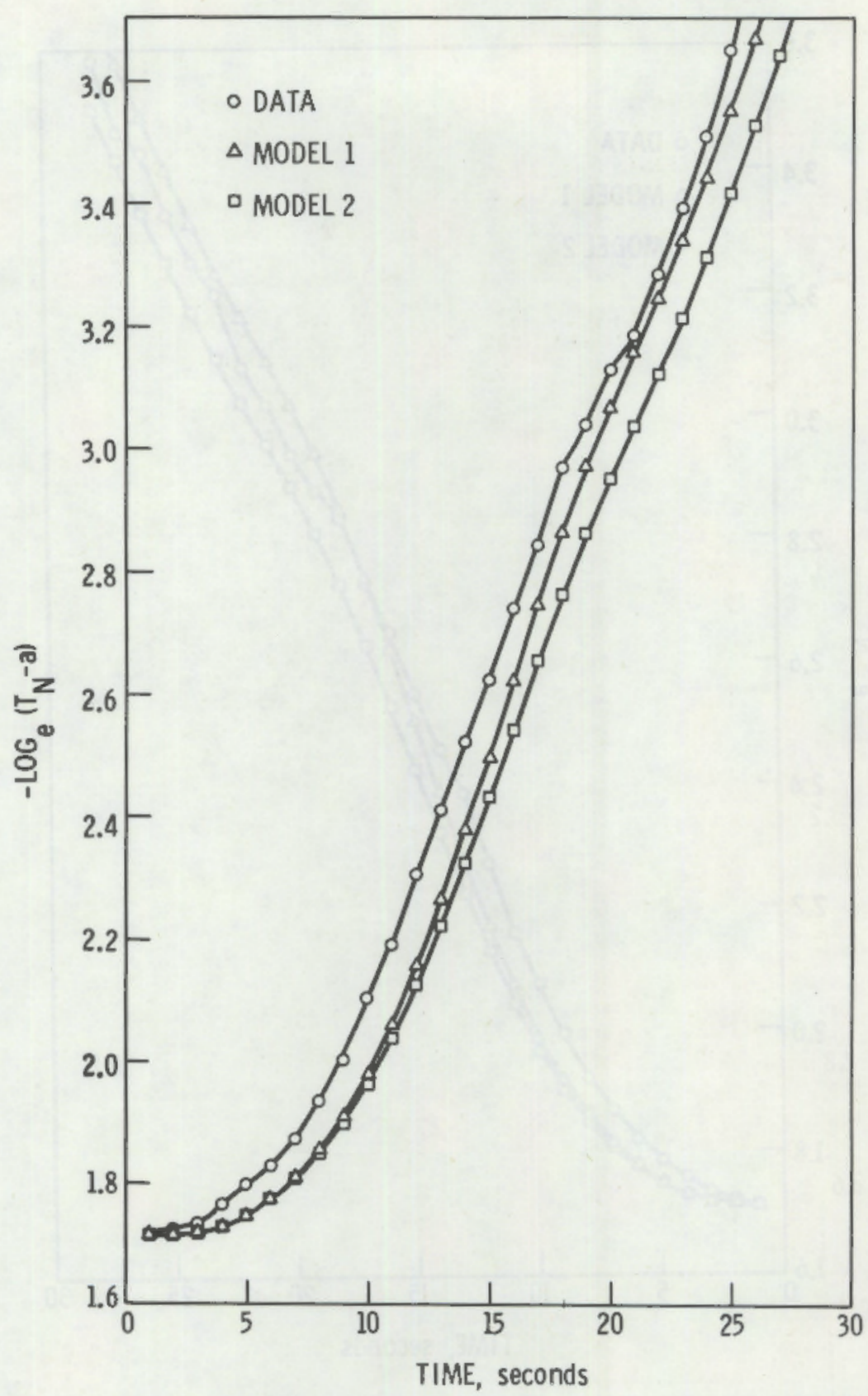

FIGURE 44. Models 1 and 2 Compared to Rod 4 Data (Upper Thermocouple) for Run 141 


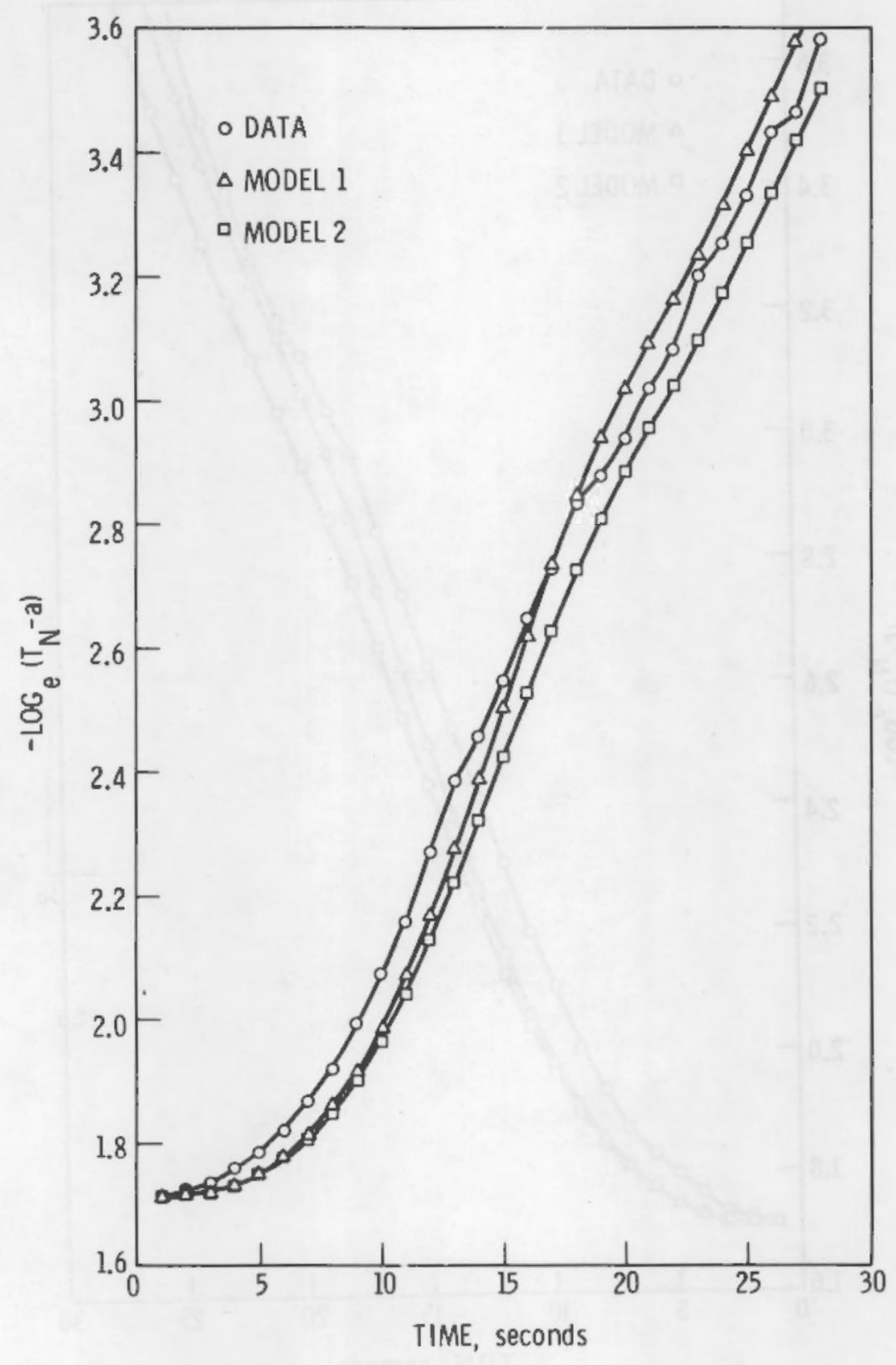

FIGURE 45. Models 1 and 2 Compared to Rod 5 Data (Lower Thermocouple) for Run 141 


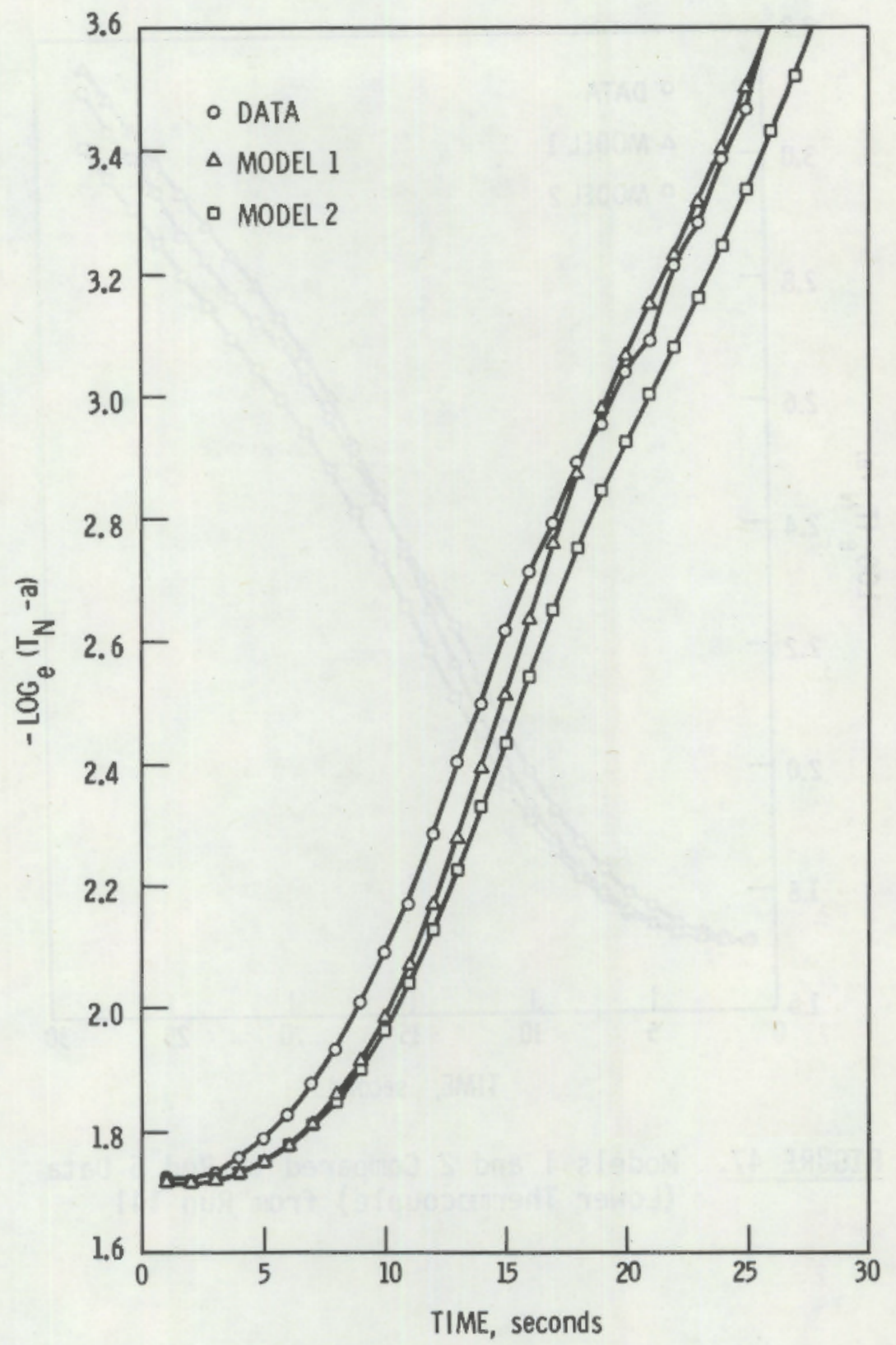

FIGURE 46. Mode1s 1 and 2 Compared to Rod 5 Data (Upper Thermocouple) for Run 141 


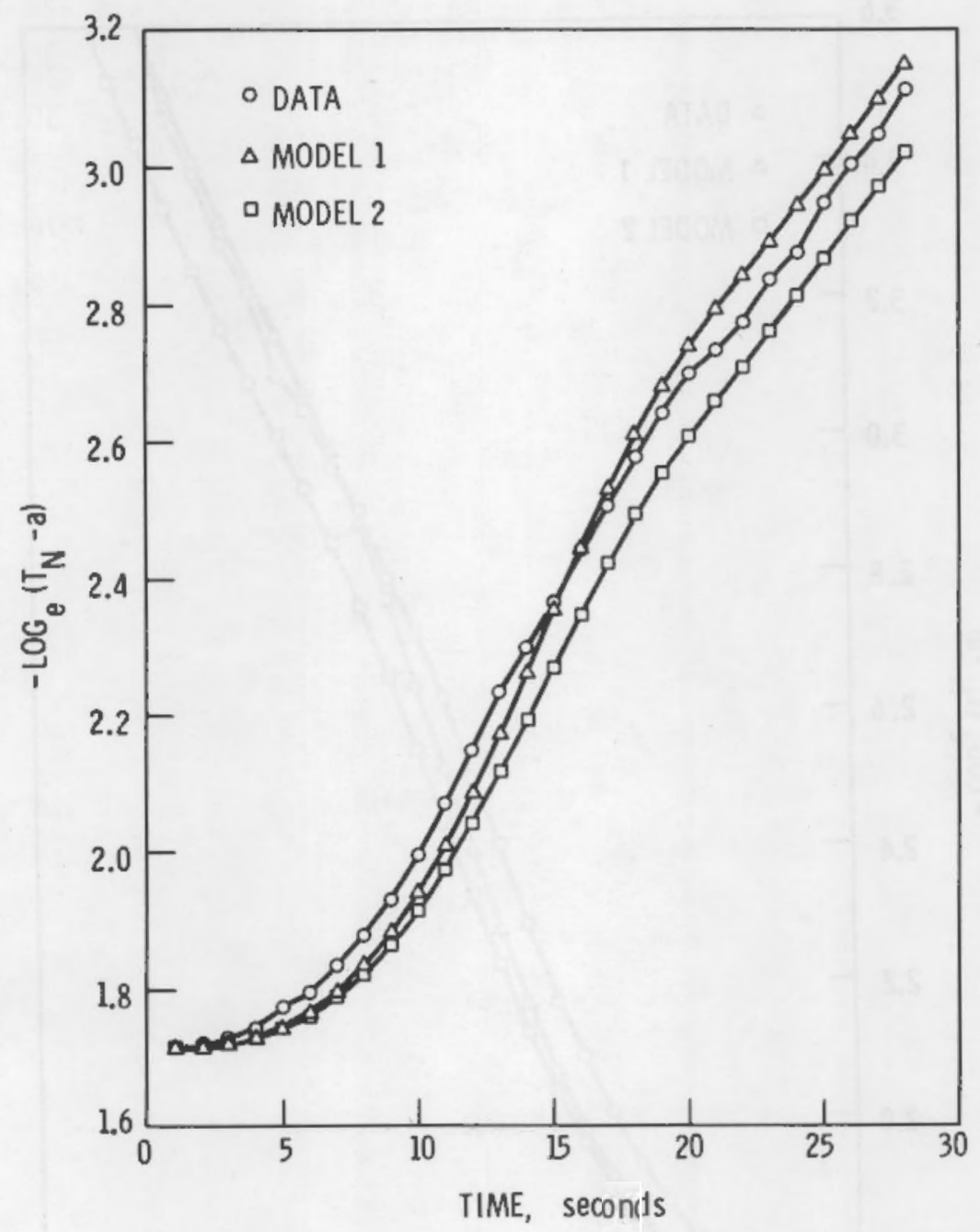

FIGURE 47. Models 1 and 2 Compared to Rod 6 Data (Lower Thermocouple) from Run 141 
TABLE 10. Thermocouple Positions and Fill Gas

Compositions for IFA-513 Fuel Rods

Thermocouple Rod Number

1
2
3
5
7
8
9
10
11
12

Number

$\begin{array}{ll}1 & \text { Lower } \\ 1 & \text { Upper } \\ 2 & \text { Lower } \\ 3 & \text { Lower } \\ 4 & \text { Lower } \\ 4 & \text { Upper } \\ 5 & \text { Lower } \\ 5 & \text { Upper } \\ 6 & \text { Lower } \\ 6 & \text { Upper }\end{array}$

Initial Power/Temp. for Run 141, $\mathrm{kW} / \mathrm{m} /{ }^{\circ} \mathrm{C}$

$30.7 / 1081$

$39.5 / 1262$

$30.3 / 1012$

$29.6 / 1011$

$29.4 / 1051$

$33.4 / 1187$

$29.7 / 1055$

$33.8 / 1172$

$30.4 / 1222$

$35.5 / 1392$

(a) All gas at $0.1 \mathrm{MPa}$ pressure at room temperature, except rod 2, which was at $0.3 \mathrm{MPa}$. 
APPENDIX

DESCRIPTION OF THE MWRAM CODE 


\section{APPENDIX}

\section{DESCRIPTION OF THE MWRAM CODE}

This appendix describes the small computer code MWRAM, which has been specifically written to predict centerline thermocouple response to small rapid power decreases (no more than 20\%). The main part of this program is the solution of coupled radial nonlinear transient heat transfer equations for fue 1 and cladding. The solution technique used is the method of weighted residuals (collocation technique) used in GAPCON-THERMAL-3. A full exposition of the solution routine is to be found in the GAPCON-THERMAL- 3 code description ${ }^{(4)}$ and will not be repeated in great detail here. This appendix will describe the various modules of MWRAM and their function. Input and output will also be described. A listing of the code and a sample problem is provided.

\section{OVERALL DESCRIPTION}

The program MWRAM calculates initial-value and transient fuel/cladding temperatures and thermocouple response for a given axial location on the fuel rod. Required input includes the fuel and cladding geometry, and "flux depression" function, initial/final steady-state values of center temperature and power, fuel density and film coefficient. The relative power history must also be input. The major parameters contained in the code (laboratory fuel conductivity, cladding conductivity etc.) are listed in Table A-1.

TABLE A-1. Major Parameters/Functions Inherent in MWRAM

\begin{tabular}{|c|c|c|}
\hline $\begin{array}{l}\text { Assumed } \\
\text { Form or Value }\end{array}$ & $\begin{array}{l}\text { Units } \\
\text { (In MWRAM) }\end{array}$ & $\begin{array}{l}\text { Reference } \\
\text { Number }\end{array}$ \\
\hline Lyons form for $95 \%$ TTD $\cup 0_{2}$ & $W / \mathrm{cm}-\mathrm{C}$ & 7 \\
\hline $\begin{array}{l}\text { Data of Conway and Feith } \\
\text { fit by Bates }\end{array}$ & $j / g-{ }^{\circ} \mathrm{C}$ & 9 \\
\hline Data of Scott for Zircaloy-4 & $\mathrm{W} / \mathrm{cm}-{ }^{\circ} \mathrm{C}$ & 8 \\
\hline $\begin{array}{l}\text { Data in Tong \& Weisman } \\
\text { for Zircaloy }\end{array}$ & $\mathrm{j} / \mathrm{g}^{\circ} \mathrm{C}$ & 11 \\
\hline $\begin{array}{l}\text { Recormendation of Haiden } \\
\text { Staff }(1.5 \mathrm{sec})\end{array}$ & sec & -- \\
\hline
\end{tabular}


Either one of two calculational models may be selected. These correspond to the "Model 1 and Model 2" discussed in the text page 31. For Model 1, the Lyons (95 \%TD) conductivity is assumed, and the gap conductance is found (in subroutine INIT) as a linear function of fuel volume average temperature. For Model 2, a constant gap conductance is assumed and a "crack factor" on the fuel conductivity is found (in INIT) as a linear function of fuel volume average temperature. The default conductance used in Model 2 is the initialvalue conductance from Model 1 . However, with a slight programming change, the user can specify any (physically reasonable) conductance value.

The program first reads general input, then the input for the specific use; then model parameters are calculated, and the solution for the initial steady-state temperature distribution is obtained. Then the power history is read in and the transient temperature response is calculated. The present logic assumes that the relative power history is the same for all cases of a given run.

The program consists of the following modules, subroutines, and functions:

Main Program - $\quad$ Reads and outputs in input, sets up the steady-state conditions, performes the transient calculations and outputs the results.

Subroutine INIT - Finds the model parameters and solution of the $\mathrm{kdT}$ equation and outputs results.

Subroutine SIMQ - Solves the 10 simultaneous collocation equations.

Subroutine STAT - Finds slope and standard deviation of calculated normalized temperature between two points (linear runs only).

Subroutine TCDR - Returns conductivity and its derivative (Lyons form for $\mathrm{UO}_{2}$ ).

Function TERP - Performs linear interpolations to get temperature dependent parameters.

Function RKINT - Finds $\int k d T$ for specified limits for Lyons $\mathrm{UO}_{2}$ conductivity function.

Function CKINT - Finds $\int k d T$ for Zircaloy cladding. 
The main program will be described briefly. But first, note that MWRAM as written is not a general purpose code that can simulate all kinds of transients (in particular, reactor scram) because:

1. The models assume linear variation of conductance/crack factor with volume average temperature. This approximation is acceptable over a small power range, but not over a large one.

2. Fuel/cladding dimensions change appreciably during large power changes, which affects the heat flux; this is not reflected in MWRAM calculations.

\section{MAIN PROGRAM}

Both steady-state and transient temperatures are found by the collocation technique. In this method, trial solutions are formed for the temperatures as a power series of radius:

$$
\begin{aligned}
& T_{f}=\sum_{i=1}^{6} B_{i} r^{i-1} \\
& T_{c}=\sum_{i=7}^{10} B_{i} r^{i-7}
\end{aligned}
$$

where $T_{F}$ and $T_{C}$ are fuel and cladding temperatures, and the $B_{i}$ are unknown.

The unknown $8_{j}$ are found by forcing the trial solutions to satisfy the four boundary conditions and forcing the appropriate heat transfer equations to be satisfied at four internal points in the fuel and two in the cladding. [The exact location of these collocation points is recommended by

Finlayson, (5) and the points are designated RC in MwRAM. (a) $]$

The above conditions generate 10 simultaneous equations. The known coefficients of these equations $A(I, J)$ are various powers of $R C$; the known

(a) The $\overline{R C}$ are normalized to the cladding outer radius so that the series expansions are convergent. 
sides (right-hand sides), B(I), involve rod power plus the nonlinear terms in the heat transfer equations, which are estimated from the temperature solution of the previous iteration or time step. Subroutine SIMQ solves these equations and returns solutions for $B$ (I) the expansion coefficients. The main program first reads the run and case variables, and then calls INIT, where the initial/final fuel surface temperatures are calculated, using both Model 1 and Model 2. These surface temperatures are found by solution of the $\int k d T$ equation.

Then the model parameters are found (slope and intercept for gap conductance or crack factor as linear functions of volume average temperature). The main program then establishes estimated values of initial steady state temperatures to begin iterative solution of the initial steady-state temperature distribution. These estimated values involve parabolic variation of fuel temperatures (from the input center temperature to the INIT-calculated fuel surface temperature) and linear variation of the cladding temperature.

Once the steady-state initial temperatures are found for all 10 collocation points, the transient temperature calculation begins. There is no iteration in the transient calculation. The power cards are assumed to be normalized power at every 1 second mark. Interpolation is performed for all time steps between the 1 -second intervals. The time and normalized temperature are output once every second.

\section{INPUT INSTRUCTIONS}

The user could probably figure out correct input instructions from the program listing. For convenience the following list of input instructions are also included: 
CARD 1 - Run Variables (FORMAT I4,6X,5F10.4, 9X, I2, I5)

\begin{tabular}{|c|c|c|c|}
\hline Variable & Card Columns & Meaning & Units \\
\hline NN & $1-4$ & Number of time steps & -- \\
\hline TSTEP & $11-20$ & $\begin{array}{l}\text { Time step size (must } \\
\text { be integral subdivision } \\
\text { of } 1 \mathrm{sec} \text { ) }\end{array}$ & $\mathrm{Sec}$ \\
\hline
\end{tabular}

DENS 21-30 Fuel pellet density Fraction of theoretical density

CARD 1 - Run Variables (FORMAT I4,6X,5F10.4, 9X, I2, I5)

\begin{tabular}{|c|c|c|c|}
\hline Variable & Card Columns & Meaning & Units \\
\hline DI & $31-40$ & Pellet inner diameter & M \\
\hline DCO & $41-50$ & $\begin{array}{l}\text { Cladding outer } \\
\text { diameter (hot) }\end{array}$ & M \\
\hline DCI & $51-60$ & $\begin{array}{l}\text { Cladding inner } \\
\text { diameter (hot) }\end{array}$ & M \\
\hline KBIG & $70-71$ & Number of cases & -- \\
\hline ISTAT & $72-77$ & $\begin{array}{l}\text { Signal for statistical } \\
\text { curve fitting }\end{array}$ & -- \\
\hline
\end{tabular}

CARD 2 - Run variables (FORMAT 5F10.4)

\begin{tabular}{|c|c|c|c|}
\hline Variable & Card Columns & Meaning & Units \\
\hline $\mathrm{AZ}$ & $1-10$ & Coefficients of heat & \\
\hline$B Z$ & $11-20$ & generation rate, & \\
\hline $\mathrm{CZ}$ & $21-30$ & $\begin{aligned} Q & =A Z+B Z r^{2} \\
& +C Z r^{4}\end{aligned}$ & Arbitrary \\
\hline HW & $31-40$ & $\begin{array}{l}\text { Film heat tranfer } \\
\text { coefficient }\end{array}$ & $W / m^{2}-C$ \\
\hline TCOOL & $41-50$ & Coolant temperature & ${ }^{\circ} \mathrm{C}$ \\
\hline
\end{tabular}


CARD 3 - Case Card (FORMAT 5F10.4, 3I5)

\begin{tabular}{|c|c|c|c|}
\hline Variable & Card Columns & Meaning & Units \\
\hline DF & $1-10$ & $\begin{array}{l}\text { Fuel outer diameter } \\
\text { (hot) }\end{array}$ & M \\
\hline POW (1) & $11-20$ & Initial Power & $\mathrm{kW} / \mathrm{m}$ \\
\hline \multirow[t]{2}{*}{$\mathrm{POW}(2)$} & $21-30$ & Final Power & $\mathrm{kW} / \mathrm{m}$ \\
\hline & CARD 3 - Case & Card (FORMAT 5F10.4, 3I5) & \\
\hline Variable & Card Columns & Meaning & Units \\
\hline$T(1)$ & $31-40$ & Initial Center Temp & ${ }^{\circ} \mathrm{C}$ \\
\hline$T(2)$ & $41-50$ & Final Center Temp & ${ }^{\circ} \mathrm{C}$ \\
\hline IRD & $51-55$ & Rod Number & -- \\
\hline ITF & $55-60$ & $\begin{array}{l}\text { Thermocouple Number } \\
\text { calculational }\end{array}$ & -- \\
\hline IMOD & $60-65$ & Model number ( 1 or 2 ) & -- \\
\hline
\end{tabular}

\section{POWER. HISTORY CARDS (FORMAT 2F10.4)}

The time and normalized power (once per second) should be input on the indicated format.

\section{LISTING OF MWRAM}

The following pages show a listing of MWRAM. Please note that this version has been written for readability, and has not been optimized for computational efficiency. The program was developed on a CYBER - 6600 and contains some non-AINSI FORTRAN statements. 
PRUGHAM MWRAM(INRUT, UUIPUT, TAPLSaINPUT, TAPCOXOUTPUT, PUACH)

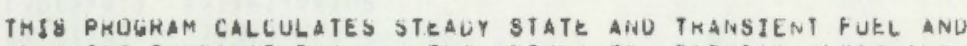
CLADUING TEMPEHATURES BY THE METMUD OF MELGHTED MESIUUALS. HEQUIREO INPUTS ARE THE INITIAL ANL FITAL CENTERLITIE TEMPEKAIURES

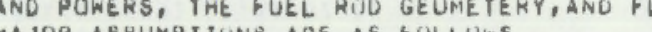

FUEL CUNLUCTIVITY - LYUNS FURM RUR 9S PER GT. TD UUZ PELLEIS,

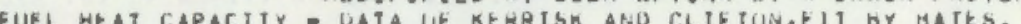

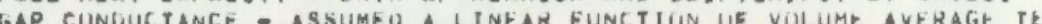

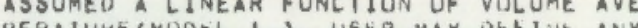

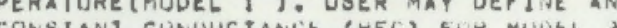

FILM CUEPFICIENT - USER INPUT AND CONSTANT.

INPUT AND OUTPUT VARIAULES ARE IN SI UNITS, ALL INTERNAL CALLULA

TIONS ARE UONE IN METRIC ENGINEERING UNITS.

MAJUR INPUT VARLAGLES AND UATA

( a INDICATES USEK INPUT)

$0=A Z+\forall Z \# R * 2 Z+C Z * R * * 4, R$ IN CM.

\#AZ,UZ,CZ - CUNSTANTS SPECIFYING RAUIAL HEAT GENERATIUN

C(I), TC(I) - CLADDING CUNDUCIIVITY AND ASSUCIATES TEMP, (W/CM=6)

WDENS - FUEL DENSITY (FRACTION TD)

MCI,DCO - INPUT CLADOING ID, OD (M)

-OT,DF - INPUT FUEL 10,00 (M)

HCAPT(I), TC ELLADDING HEAT CAPACITY ANU ASSOL. TKMP(J/G-C)

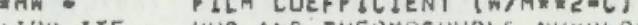

IRU, ITF - KUD AND THERMUCUUPLE NUMUERS.

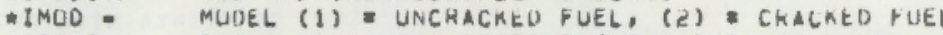

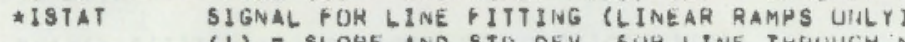

(1) = SLOPE AND STO DEV. FUR LINE THRUUGH MUKM. TEMP.

PUINTS FRUM SSTART TO SSTUP IS UUIPUT.

aKEIG (U) = NO CALCULATION

MKEIG

-NN - - NUMEER uF tIME SIERS

- ingtial and FINAL PUAERS (KN/M)

PUINTS IN FUEL ANU LLADUING (NLIKMALIZED?

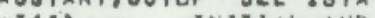

FINAL STEADY-STATL'TEMPERATHKES (C)

- cuolant temp. (c)

DETHE IIME CUNSTANT (SEC**-1)

"TIME SIEP SIZE (SEL)

(MUST UE AN INTEGRAL SUAOIVISION UF I SECGNO)

MAJUR CALCULATIUNAL YARIAGLES

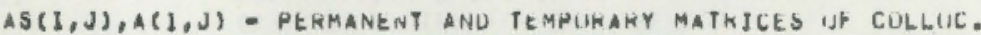

EGUATIUN CULEFICIENTS.

ALPM(I) - DIFFUSIVITIY TIMES TIME STEP AT CLLLIC PTS.

H(1) - EXPANSIGN CUEFRICIENTS UF CLLLC, SULUTIUNS.

DENC DENSIIY $(G / C M * * 3)$

DKDT CUNUUCTIVITY DEKIVAIIVE $(W / C M * C * \# 2)$

FT KATIO OF CURRENT POWEK TO INITIAL PUAEM

W(1) CURAENT PUAEN (N/CM*B3) AT COLLOC. PIS

(IIMES KD*\#2) 


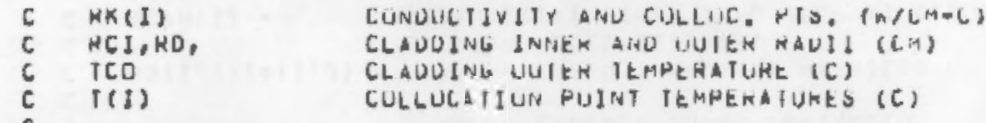

CULGUCAIIUN PIJINT TEMPERAFURES (C)

UIMENSIUN A $(10,10), d(10), k K(10), B,(10), A D(10,10), 1(10), \mathrm{Cl} /(6)$

$\times R C(10), T U L .0(10), 0(10)$, QULU $0(10), A L+(10), R T L(4)$

UIMENSIUN TC(S), CCS), MCAPT(S)

DIMENSION INSVR IOOD

DIMENSION FIX(1000)

DIMENSIUN PUN(2), TCU(2), ICLAU( (2), KMULT(2)

DIMENSION KKH(IO)

DATA $(R K K(I), I=1,5), 0,2,0.3,0,4,0,0,0,01$

DATACC(1), Iस1,5)/.1300,.1431,.1521, .1640,.1749/

DATACTC(I), $J=1,5) / 100,1200.1300,1400.1500 .0$

DATACHCAPT(S), 1

DATA $(K T(1), 1=1,0)$

$0.0,0.0694310,0.33000948,0.069440520$

DATARTC(1), $1=1,4) / 0,0,0.2113248,0,78007,1.01$

READ MAIN VAKIABLLES

READ $(5,00)$ NN, TSTEP, OENS, DI, DCU,DGI,KEIL, ISTAT

READ $(5,10) A Z, G Z, C Z, H W, T C O D L$

HW $\mathrm{HW} / 10000$

UU 1111 IUJG' $1, K \in I 6$

C ZERU PERMANENT CUEFFICIENT aRKay

$$
\begin{aligned}
& \text { UU } 122 \mathrm{I}=1,10 \\
& \text { AS(1)J) =0.0 }
\end{aligned}
$$

123 CUNTINUE

S CUNTINUE

$$
\text { IF IISTAT, INE, O) WHITK }(0,18) \text { SSTAHI, SSTUP }
$$


$\mathrm{KCI}=\left(0 \mathrm{C} 1 / \mathrm{z}_{0}\right) \times 100$

PLW(1) P POW(1):10。

POW (2) $=$ PUW (2) 110

OENC = UENS\$10.46

OR DIJDCO

C CALL INITCPUW,T,TCU,AZ,BZ,CZ,RK,RI, CHN, AATT, UUTT, SLUP, HINT

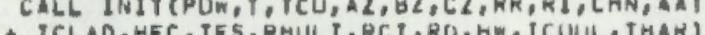

C INITIALIZING LUUP-ODEFINE INITIAL CULLOCATIUN POINI

TEMPERATURES ANU VOLUMETKIC MEAT GENERATION.

130

$K N=P U W(1) * R D * 2 /(C H N * 2, * 3.14159)$

OO $100 \mathrm{~K}: 1,10$

IF $(K, G \mathrm{~T}, 0)$ G

$R C(K)=0 I / D C Q+R T(K) *((D F-v 1) / U C L)$

$K M=R C(K) \backsim R D$

$T(K)=(T(1)-T F S) *(1,-(N M / R R) * \star 2)+T H S$

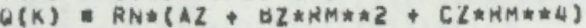

QOLO $O(K)=Q(K)$

60 To 100

$101 \mathrm{RC}(K)=(1,0)=D C 1 / D C O)+H T C(K-6)+D C 1 / D C O$ SLOPE $=(T C L A D(1)-T C O(1)) /(R D-F C I)$

$T(K)$ \& SLOPE $\#(H D=R M)$ + ICO(1)

100 CONTINUE

$00600 \mathrm{~J}=1,6$

$(J-1) * R C(1) * *(J-2)$

$A S(6, J)=R[(0) * *(J-1)$

$600 \mathrm{CON}$

$500 \pm=2,5$

As(1,

$A S(1,3)=4.0$

AS $(1,4)=9,0$ \#C (1)

AS $(1,5)=10,0 * R C(1) * *$

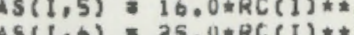

500 CONTINUE

c

SET UP CLAD EQUATIUNS

ou $601 \mathrm{~N}=7,10$

$A S(0, J)=-R C(7) * \omega(j=7)$

$A S(7, J)=(J-7) * A C(7) * *(J-8)$

$\Delta S(10, J)=1,0$

601 CONTINUE

UU $504 t=8,9$
A $5(1,7)=0,0$ 
$A S(1,8)=1.0 / K C(1)$

AS $(1,9)=0.00)=9.0 \pm H C(1)$

175

504 CONTINUE

IIERATION GEGINS MERE.

DO $200 N=1,20$

180

c SET EXPENDABLE $A(I, J)$

DU $800 \mathrm{~J}=1,10$

OU $801 \quad I=1,10$

$A(1, j)=A S(1, j)$

185

GOI CONTINUE

C THSS LUUP DERINES THE RIGHT HANU SIUES (KNUMN SIUES) UF THE

140

195

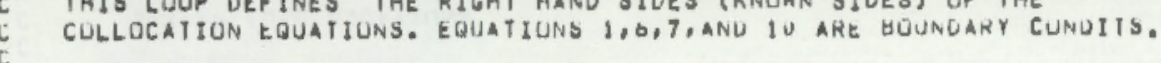

$$
\text { DO } 300 K=1,10
$$

IF $(K, G T, b)$ GO TU 301

,OKDI)

IF (IMUD, $(6,2) R K(K)=R K(K) * R M U L T(1)$

(R) UKUT = DKOI*RMULI (I)

$\forall 02$ RAC $=R N /(K C(K) * R K(K))$

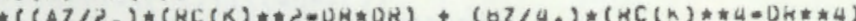

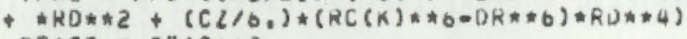

TFACT $=$ IFAC:\#Z

$E O(K)=-(D(K)+U K D T * T H A C T) / H K(K)$

IF $(K, E Q, 0) B(K)=\operatorname{PLW}(1) /(K H \# H F C$ औ. $14159 * 2$.

Go TO 300

301 TTEST $T(K)+1.0$

RKTEST \& TERP(TTEST,TC,C,S

$R K(K)$ :TERP(T $(K), T C, C, 5$

OKUT EKKTEST. RK(K)

TFAC $=-(R C(7) / R C(K)) *(R D / K K(K)) *(P(\mid K(1) /(K C D * 2 . \$ 3.14159))$

TFACT = TFAC\#\#E

$B(K)=$ OOKDT TTFACT/KK(K)

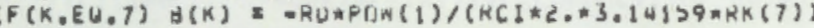

IF $(K, E Q, j U) \forall(K)=T C U(1)$

300 CONTINUE

$\forall(1)=0.0$

C SULVE THE COLLUCAIION EQUATIONS FUK b(d)

CALL SIMUS $A, B, 10)$

SUM $=0,0$

IF (1.6T.6) G0 TO 702

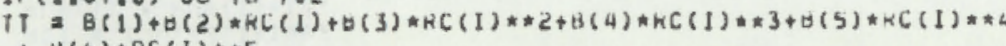

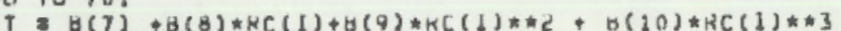

A $\operatorname{sit}(T(I)-1 T)$

$S U M=S U M+A U E L$ 
250

235

240

245

250

700 CONTINUE IF (SUM.LT, U.1) GU TO :

200 CONTINUE

1 WRITE $(0,30)$

WRITE $(0,50)$

c begin transient calculatiun

KOUNT : 0

LKNT" 0

ATEST a 1 TTSTEP

TRMULO $=1.0$

OU QUO $L=1, N$

KOUNT = KOUNT

C READ IN NURMALIZED PUWERSCONCE PER SECUNO) ANO LINEAKLY INTEKPULATE

TO GET PUWERS FOH EACH TIME STEP.

IF (KUUNT,EG,I,AND,IHIG,EL, 1) READ $(5,80)$ UUM, FTX(L) IF(KOUNT,EQ.1) FTCUR $=F T X(L)$

FT = (FTCUR OFTPAST)\#PLIJAT(KUUNT)HTSTEP \& FTPAST

TFAV $*(T(1)+T(b)) * 0.5$

IF (IMOU,EG, 1) MFC. AATT \& BBTT ETFAV

$A(1,1)=0.0$

$A(7,1)=0.0$

STEP G TSTEP

STE " ISTEP*L

$A(6,1)=H F C$

260

SET UP FULL EQUATIUNS

DO $1000 K=1,0$

$A 6(6, K)=\operatorname{HFC} * R C(0) * *(K-1)+(R K(0) / R U) *(K-1) * R C(0) * *(K-2)$

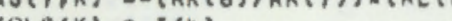

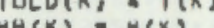

1000 CONTINUE

270

C SET UP CLAD EQUATIIINS

$D D 1001 K \approx 7,10$
$A S(6, K)=-H F C * K C(7) * *(K-7)$

As $(10, K)=H W+(R K(10) / R O) *(K=7)$

$T O L D(K)=T(K)$

275

$\Delta(k)=\theta(k)$

1001 CONTINUE

280

C SET UP RIGHT-HAND-SIOES UF CULLUCATIUN EGUATIONS.

OO $1100 K=2,10$

$A(1, K)=A S(1, K)$

$A(b, K)=A s(b, K)$

$A(7, K)=A S(7, K)$

285

$(10, k) \approx A(10, k)$ 
If $(K, G T .0)$ GO TI 1101

CALL TCOK(I $(K), K K(K), U K L)$

RMULT (2) XRINT+SLUP \#THA

ONDIAHMULT(

IF (IMOD,KO, 2) KK $(K)=K K(K) * K M U L T(2)$

$1002 \operatorname{IF}(K, E G, b)$ GO TU 1100

SUM $=0.0$

3,0

$T R M=\theta H(M) *(M-1) * K C(K) * *(M=3)$

SUM S SUM

1400 CÜTINUE

TEMP TULO $(K)$

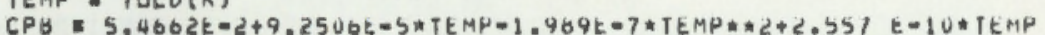

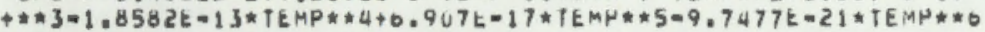

$C T=C P B * 4.186$

$A L F(K)=\operatorname{STEP} * R K(K) / C D E N C * C T \# R U * \approx 2)$

60 TO 1102

1101 DENCC $=6.57$

RK (K) $=$ TERP (TOLU $(K), T C, C, 5)$

IF $(K, E Q, 7, O K, K, E Q, 10)$ GO TO 1100

TTEST $\Rightarrow$ TULU $(K)+1.0$

RKTEST = TERP(TTESI, TC, C,S

DKUT D RKTEST - RK(K)

1103 SUM $=0.0$

$001401 M=0,10$

$T R M=B B(M) *(M-7) * K C(K) * *(M-8)$

SUM $=S U M+T R M$

1401 CONTINUE

CT * TERP(TOLU(K), IC, HCAPT, 5 )

$A L F(K)=\operatorname{STEP} \# R K(K) /(O E N C C * C T * K D * 2)$

1702 CONTINUE

C CORRECT COEFFICIENTS at CULLOCatiUn PUINTS.

$001200 \times 1,10$

$M G=1$

IF $(K .61 .0) M G \equiv 7$

(

IF $(K, G T, 7, A N D, M, L T, 7) A(K, M)=0,0$

IF (K,LT.O.ANO.M.GT, G) $A(K, M)=0.0$

1200 CONTINUE

1104 TFACT a SUM*\#2

$Q(K)=Q U L D(K) * F T$

$\theta(K)=-(Q(K)+D K D T * \mid F A C T) / K K(K)=\mid U L D(K) / A L F(K)$

1100 CONTINUE

$\forall(1)=0.0$

$B(0)=0,0$

$\forall(7)$.

$B(10)=$ TCUDL *HW

CALL SIMQ $(A, B, 10)$

DU $1300 \square=1.10$

$1 F(1.67 .6)$ Go iu 1301

$11 * \theta(1) * \forall(2) * R C(I)+B(3) * R C(1) * * 2 * \forall(4) * R C(I) * * 3+B(5) * R C(1) * * 4$

$x+B(6) * R C(1) * * 5$

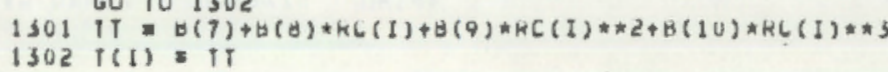


5

10

\#DEGK INIT

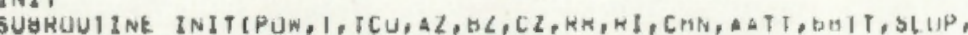
- RINT, TCLAD, HEC, IFST, RMULT, RLE, RL, MR, TLUUL, TOAR]

THIS SUBRUUTINE PINOS THE MUUEL PARAMEIEHS PUK MIJUELS I AND 2. IN MUOEL I TME CONOUCTANCE IS A LINEAR FUIYCIILIN IIT TME VUL AVG, BUEL TEMP. IN MUOEL 2 TME LUNDUCTIVITY MULTIPLIEK IS A LINEAR FUNCTIUN UF FUEL VOL AYG. TEMF.

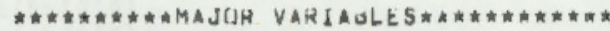

AATT,BGII SLOPE AND INTERCEPT FUR CUNUUCTANLE

CHF FLUX DEPRESSIUN FACTOR FUK CENTER TEMPEKAIURE

CKINT CONUUCTIVITY INIEGGKAL FUR TAE CLAUDING (H/CM)

HF GAP CUNOUGTANGE $(W / C M * 2-C)$

CONDUCTIVITY INIEGRAL. FUR THE PUEL (M/CNI)

- CAACK PACTUR UN FUEL CUNDULTIVIIY (MUDEL.

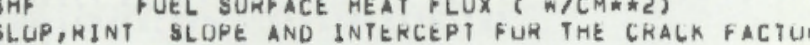

TCLAD INNER CLAUDING SURBACE TEMP. (C)

THAR VOU AYG. PUEL TEMP.

IFS FUEL SURFACE IEMP (C)

UIMENSION PON(2), TCU(z), MF(2), KMULT(z), TCLAU(z), THAH(z) OIMENSIUN T(10), IR(2, 2$]$ WRITE $(6,50)$

C GET CHF

$C \quad C H E=(A Z / 2) *,(H R * R R=R I * R I) *\left(B Z / \theta_{0}\right) *(K R * \# 4-K I * \# 4)$ * $+(C Z / 10) *,(H A * * 6-K I * 0)$

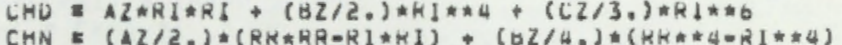
+ (CZ/O, $) *(R R * \# \div=H I * * 6)$

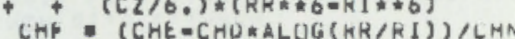

C SULVE PDR +UEL SURFACE TEMP. (MUUEL 1)

GET CLADUING INNER SURPACE TEMH.

DU 107 I $=1$.

CH: CHF\#PUW'(I) 112.5

TEST $\approx 200$.

$00207 \mathrm{~J}=1,20$

TEST: TEST $\$$ TOU.

TINT : RKINTCT(I), TEST)

FE(IINT.LT.CH) GU IU 200

207 CONTINUE

208 TEST \# TEST - 100.

VO $307 \mathrm{~J}=1,10$

TEST" TEST + 10 .

IINT \#KINT(TII), IEST)

IF (TINT.LT,CH) GU TU 308

$00407 \mathrm{~J} \cdot 1.10$ 
$\triangle 0$

ob

70

ISNT : RKST T(I), IEST

IF(TINT,LI.CH) GU IU WUO 407 CUNTINUE

c

SHF IPOA (I) $/(3.14154 \# K R * 20)$

TCO(I) POW(I) (3.14159kC, \#HW\#KD) + TCUOL

APUW : PUN(I)\#ALUGS(RD/RCL)/6.283185

$T^{\prime K}=\mathrm{TCO}(1)$

IKE $=10$.

IKF 100 J 1.0

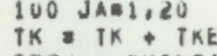

CPON = CKINT(TK, TCD(1))

IF (APUWOCPON,LE.O,)GO TU 101

100 CUNIINUE

IK $T K=T K E$

TKR N TKETTKF

Qu 105 L $L=1, K$

IK $T K+I K F$

CPOW $=$ CKINITIK, TCO(I))

IF(APON-CPOW,LE, O.)GU TO 103

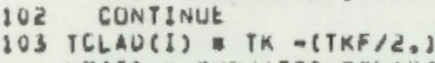

MF(I)

TPAR(I)

TR(1,I): TFS

107 CUNTINUE

C CALCULATE mUdel 1 PARAMETERS

10

105

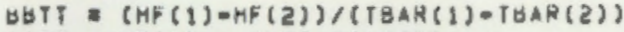

AATT " MF(L) - HUTTMTBAR(I)

C GET FUEL SURFACE TEMP. (ADOEL E)

C MFCOMF (1)

UD 507 I 1,

TFS $=T C L A D(1)+P Q W(I) /(3.14159 * H+C * H K * 2$,

It (I.EU,I) IFS! I THS

IINT = RKINT(T) D, TPS

RMULT(I)*(TOW(I)*CHF)/(TINT*4,*3.1415920)

TBAAC(I): $(T(T)+T F S) / 2$.

$T R(2, I)=T F S$

co fukmar(3F 10.4$)$

c Calculate movel 2 pahameters

SLUP = (KMULT(1))-AMULT(2))/(THAR(1)) I UAR(2))

KINT = RMULT(1) - SLUP: TBAR(1)

WRIIE $(6,10)$

UPUW BPOW(1)

TPQW

$Y H E M H E(3) * 10$. 
115

IHAF $\#$ AHF (1)*10.

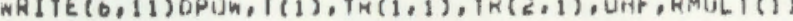

WRITE $(6,50)$

WATIE $(0,12)$ EPUA, T (2), TM( 1,2$), T K(2,2), V H F, R M U L T(2)$

120

URN

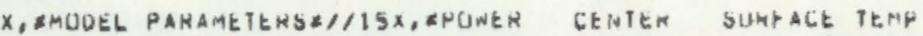

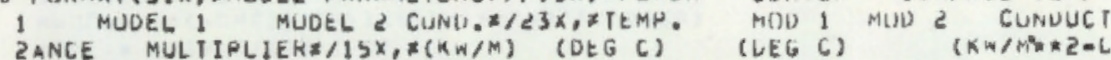
3) (2)

1 FORMAT(5x, 5 INITIAAL $\left.4 x_{0}, F 4,1,4 x_{0}+5,0,2\left(3 x_{0}, 50,1\right), 4 x_{0}+0,4,0 x, 20,4\right)$

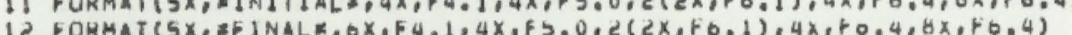

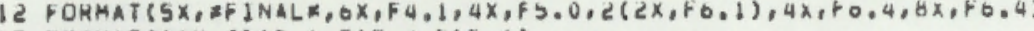

7 FURMAT $10 x, 3515,1, F 15,4, Y 15,1)$

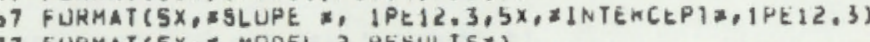

7 FURMAT(5X, * MODEL 2 RESULTS $x$ )

5U FURMATS

END 
\#UECK ICOR

GUBROUTINE TCOKIT,RK, DKUT)

Sot $=15 *(1+275,1) * 3$

WMOT $=-30.24 /(T+402.4) * 2+3, * 0.1256 t-13 *(T+275) * *$.

RETURN 
PINCIION CKINT

- UECK CKI Th

FURETION CKINI $(X, Y)$

CKL1 $=0.1326$

CK2 $=0.000020843$

CKJ $=0.00000024643$

$=C K !(x \in Y)$

$B=C K 2 *(x * \# 2-Y * * 2) / 2$.

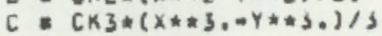

CKINI $A+B+C$

END 
MDECK STAT

SUBROUTINE STAT(TN, XLLI, XHI, SA, SO,N)

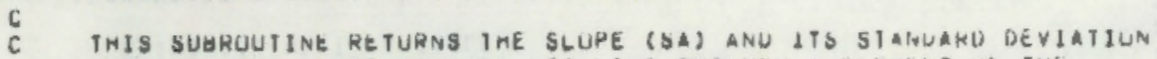

(SA) FUR THE LEASI SQUANES FII LIME IHRUUGH SFGMENT UR THE

NORMALIZEO IEMPERATURE VS. TIME PUINTS.

OLMENSIUN TN(1UO)

C INITIALIZING THE CUNSTINIS

c

$x=x \operatorname{Lim}=1$
$\operatorname{sum} x=0,0$

$\operatorname{sum} \times 2=0.0$

SUMY $=0.0$

SUMY $2=0.0$

SUMXY:0,0

DO $10101, N$

$x=x+1$

$\operatorname{SUM} x \geq \operatorname{sum} x+x$

$\operatorname{sum} \times 2=\operatorname{SUM} \times 2+x * * 2$

SUMY $=8 U M Y+T N(t)$

SUMY $2=S U M Y Z+T N(I) * \#$

10 CUNTINUE

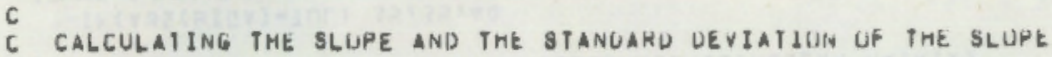

Fn FlUat(n)

SP - SUMAY - SUMX*SUMY/FN

$9 S X \approx \operatorname{SUMX}=$ SUMX SUMX/FN

SA S SP ISSX

- SUMY\#SUMY/FN) -SA\#SA\#SSX

$S B=S G R T(S S R E S /((F N-2) \# S S X)$.

90 FURMAT(L,Z LEAST SUUARES SLUPE $= \pm, E 10,40$

Iz STANUAKD OEVIAIIUN $=z, E(0.4)$

RETURN 
*DECK SIMU SUHROUTINE SIMW(A, d,N) DIMENSIUN A(1), D(1)

ronwaku solutioje

TOLE 0.0

$K \leqslant \geq 0$

$J \mathrm{~J}=-\mathrm{N}$

NO $65 \mathrm{~J}=1, \mathrm{~N}$

$3 Y \geq 3+1$

$J j \in J N+N+$

HI

DU $30 \quad I=J, N$

C SEARCH ROR MAXIMUM CUERFICIENT IN COLUMN

$$
[J] I T+1
$$

IF (ABS(BIGA)-AUS(A(IJ))) $20,30,30$

ODIGAaA(IJ)

IMAXTi

TEST FUR PIVDT LESS THAN TOLEHANCE (SINGULAR MATHIX)

IF(AUS(UIGA)-TUL) 35,35,40 35 KSAI
RETURH

$\mathrm{c}$
$\mathrm{c}$

INTERGHANGE RUWS IF NECESSARY

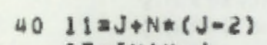
$I T=\operatorname{IMAX}-\mathrm{T}$

DO $5 U K=\mathrm{J}, \mathrm{N}$

II $12 I 1+N$

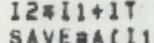

SAVEAA(I1)

$A(111)=A(12)$
$A(12)=S A Y E$

C DIVIUE EGUATIUN BY LEADING CUEFFICIENT

SU A (II) EA(II) /GIGA

SAVEEB (IMAX)

$\forall(\operatorname{IMAX}) \operatorname{BS}(J)$

\section{ELIMINAIE NEXT VAKIAGLE}

IF $(\mathrm{J}=\mathrm{N}) \quad 55,70,55$

$5516891 N *(7-1)$

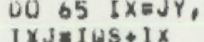

$1 x=16 x+1 x$

IT $=50$
Striw buo

SIMU 510

In 520

SIMU SSU

SIMU 540

SIMU 550

SIMU 500

Simu 570

SIMU 580

simu squ

simi b00

sime olv

sImए bद

IMO

SIMU o $\rightarrow 0$

sImu bo

SI HA 070

SIMG 080

SIMW 690

SIMU 700

SIMU 700

$\operatorname{SIMGO} 720$

SIMU 750

SIMU 740

SIMU 750

SIMU 760

SIMU 770

SIMU 780

SIMU 790

SIMG avo

SIm 810

5 IML 820

SIMid aso

SIMW 840

SIMW USO

Simu 860

SIMU 870

SIMU 800

SIMU dyo

sime 900

SIMU 9

SIMU 930

SIMU 930

stmo 90

SINW 4 bo

sinu 970

SIMW 900

SImu 990

simu 1000

Imw

SIMW1010

IMU J 030

S1 141040 
SUUROUTINE SGMG

74/74 UNI 121

$J j x=7 x y x+7 t$

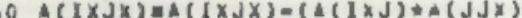

$\circ 0$

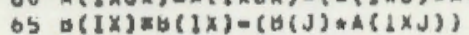

c

75
BACK SULUTIUN

$70 \quad N Y \cap N=1$

TTEN*N $301, n$

$A=I i-J$

$I \forall a N=J$

DU $80 k=1, J$

$B(1 B)=B(1 B)=A(I A) * B(I C)$

OO I I $I I A=N$ HE TURN
END

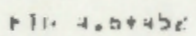

$31100 / 1401200000$

Pale 
*DECK HKIN

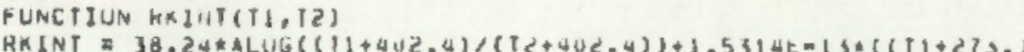

(120273 2 (1)

RETURIN

END 
MDECK TER

FUACTIUN TERH (TT,TS,TU,N)

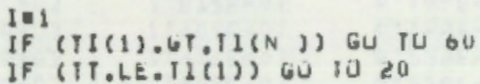

if (TP.GE.TI(N)) GO TU 30

UO 10 jel, N

IF $(T T-T I(J)) ~>0,40,10$

10 CUNTINUE

TEKP 20 TD (1)

KETURN

30 TERPETU(N)

RETURN

40 TERPETOC(J)

RETURN

5O CONTINUE

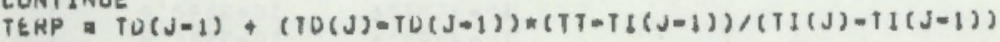

RETURN

OO IF (TT,GE.TI(1)) GU TU 20 IF (TT.LE.TISN) GO TU 30

UO 70 Jal, N

IF $(170 T$ (I) $70,40,50$

O CUNTINUE 
INITIAL DATA - HOU O IH IL MIILEL 2

I0 (M) UU (M) HEAT GENERATIUIN RAIE CUEFPICIETTS

FUEL $\quad .0017$

.01090

$A=.7545$ ba

.0112

1.3853

CLAO $\quad .01090$

.01279

MUDEL PARAMETERS

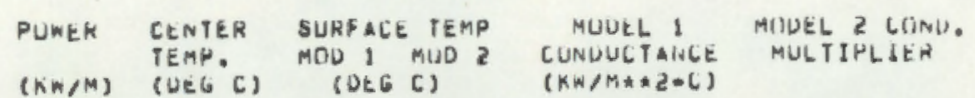

INIITAL 41.6 1575. 599.S $599.5 \quad 4.3140 \quad .9991$

FINAL 33.5 1310. 375.5 530.2 3.5901 .9233

$\begin{array}{llll}\text { IIME } & \text { CENTER } & \text { RELATIVE } & \text { NUHMALILED } \\ \text { (SEC) } & \text { (DEG C) } & \text { PUNER } & \text { TEMPERATURE }\end{array}$

$1.000 E+00 \quad 1.512 E+03 \quad 1.000 E+00 \quad 1.000 E+00$

$2.000 E+00 \quad 1.571 E+03 \quad 9.925 E-01 \quad 9.999 E=01$

$3.000 E+00 \quad 1.570 E+03 \quad 9.834 E-01 \quad 9.990 E=01$

$4.000 E+00 \quad 1.567 E+03 \quad 9.713 E=01 \quad 9.973 E=0$

$5.000 E+00$

$6.000 E+00$ T.

T.00OE+

Q.

$9.000 E+01 \quad 1.523 E+03 \quad 0.913 E=01 \quad 9.652 E=01$

$1.100 E+01 \quad 1.511 E+03 \quad B .717 E=01 \quad 9.565 E=01$

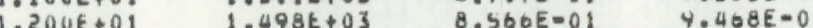

$\quad 3.484 E+03 \quad 8.415 E-01 \quad 9.303 E=01$

$1.400 E+01 \quad 0.325 E-01 \quad 9.254 E=0$

$1.500 E+01 \quad 1.455 E+03 \quad 8.264 E-01 \quad 9.145 E=01$

$1.000 E+01 \quad 1.441 E+03 \quad 8.204 E-01 \quad 9.039 E-01$

$1.700 E+41 \quad 1.428 E+03 \quad 8.174 E-01 \quad 8.938 E-01$

$1.600 E+01 \quad 1.415 E+03 \quad 8.143 E=01 \quad 8.843 E=01$

$1.900 E+01 \quad 1.403 E+03 \quad 8.090 E-01 \quad 8.755 E-01$

$2.000 E+01 \quad 1.392 E+03 \quad 0.038 E-01 \quad 8.671 E=0$

$2.100 E+01 \quad 1.382 E+03 \quad 8.023 E=01 \quad 8.591 E=0$

$2.20 u \varepsilon+01 \quad 1.372 t+03 \quad 7.992 E=01 \quad 8.318 E=0$

$2.300 E \$ 01 \quad 1.303 E+03 \quad 7.902 E=01 \quad 8.444 E=01$

$2.400 E+01 \quad 1.355 E+03 \quad 7.902 E=01 \quad 0.380 E=01$

$2.500 E+01 \quad 1.348 E+03 \quad 7.962 E=01 \quad 8.330 E=0$

$2.000 E+01 \quad 1.341 E+03 \quad 7.902 E=01 \quad 8.278 E=01$

$2.700 E+01 \quad 1.335 k+03 \quad 7.917 E=01 \quad 0.229 \mathrm{E}=0$

.800E+01 $\quad 1.329 E+03 \quad 7.902 E=01 \quad 8.165 E=01$

$\begin{array}{llll}2.900 E+01 & 1.323 E+03 & 7.672 E=01 & 8.144 E=01 \\ 3.000 E+01 & 1.319 t+03 & 7.887 E-01 & 8.107 k=01\end{array}$ 


\section{REFERENCES}

1. D. D. Lanning, B. O. Barnes and C. R. Hann, Manifestations of Nonlinearity in Fuel Centerline Thermocouple Transient Data: Implications for Data Analys is, NUREG/CR-0220, PNL-2692, Pacif ic Northwest Laboratory, Richland, WA 99352, January 1979.

2. D. D. Lanning and M. E. Cunningham, Startup Data Report for NRC/PNL, Halden Assembly IFA-513, NUREG/CR-0862, PNL-2948, Pacific Northwest Laboratory, Richland, WA 99352, July 1979.

3. G. A. Berna, et al., FRAPCON-1: A Computer Code for the Steady-State Analys is of 0xide Fuel Rods CDAP-TR-78-032, EG\&G, Inc., Idaho Falls, Idaho 83401, November 1978.

4. D. D. Lanning, C. L. Mohr, F. E. Panisko and K. B. Stewart, GAPCONTHERMAL-3 Code Description, PNL-2434, Pacific Northwest Laboratory, Richland, WA 99352, January 1978.

5. B. A. Finlayson, The Method of Weighted Residuals and Variational Principles, Academic Press, New York, NY, 1972.

6. A. L. Edwards, TRUMP: A Computer Program for Transient and Steady-State Temperature Distributions in Multidimensional Systems, UCRL-24754 Rev 3, Lawrence Livermore Laboratory, Livermore, CA 94550, September 1972.

7. M. F. Lyons, et al., $\mathrm{UO}_{2}$ Pellet Thermal Conductivity From Irradiation with Central Melting, GEAP-4624, General Electric Corporation, Sunnyvale, CA $94086,1964$.

8. D. B. Scott, Physical and Mechanical Properties of Zircaloy-2 and Zircaloy-4, WCAP-3269-41, Westinghouse Electric Corp., Pittsburgh, PA 15235, 1965.

9. J. L. Bates, 1970. High Temperature Thermal Conductivity of Round Robin Uranium Oxide, BNWL-1431, Pacific Northwest Laboratory, Richland, WA 99352.

10. R. B. Bird, W. E. Stewart and E. N. Lightfoot, Transport Phenomena, John Wiley and Sons, Inc., New York, NY, 1966.

11. L. S. Tong and J. Weisman, Thermal Analys is of Pressurized Water Reactors, ANS Publication, 1970. 
1 


\section{DISTRIBUTION}

No. of

Copies

OFFSITE

A. A. Churm

DOE Patent Division

9300 S. Cass Avenue

Argonne, IL 60439

295 Nuclear Regulatory Commission

Division of Technical Information and Document Control

7920 Norfolk Avenue

Bethesda, MD 20014

2 DOE Technical Information Center

4 W. V. Johnston

Chief, Fuel Behavior Research Branch

Division of Reactor Safety

Research

U.S. Nuclear Regulatory Comission

Washington, DC 20555

\section{ONSITE}

41 Pacific Northwest Laboratory

W. J. Bailey

J. 0. Barner

E. R. Bradley

E. L. Courtright

M. E. Cunningham (5)

M. D. Freshley

R. L. Goodman

R. J. Guenther

C. M. Hagen

C. R. Hann

P. L. Hendrick

K. A. Hsieh

D. D. Lanning (10)

R. K. Marshail $\underline{\text { ONSITE }}$

Pacific Northwest Laboratory (contd)

C. L. Mohr

C. Nealley

F. E. Panisko

P. J. Pankaskie

W. N. Rausch

R. E. Schreiber

R. E. Williford

Technical Information (5)

Publishing Coordination (2) 
